Journal of Innovative Engineering
and Natural Science

\title{
Modeling water quality parameters in the surface waters of Samanlı and Safran Rivers, Turkey
}

\author{
Ferdi Ozan ${ }^{\mathrm{a}}$, Nergiz Kanmaz ${ }^{\mathrm{b}}$, Osman Koçalc and Julide Hızal ${ }^{\mathrm{b}}$ \\ ${ }^{a}$ Yalova University, Institute of Science, Chemical Engineering Department, 77100 Yalova, Turkey. \\ ${ }^{b}$ Yalova University, Engineering Faculty, Chemical Engineering Department, 77100 Yalova, Turkey, \\ 'Yalova University, Engineering Faculty, Computer Engineering Department, 77100 Yalova, Turkey.
}

\section{ARTICLE INFO}

Article history:

Received 07 July 2021

Received in revised form -

Accepted 29 July 2021

Available online

Keywords:

Surface water

Water pollution

Physicochemical properties

Multivariate techniques

\begin{abstract}
In this study, the water quality of the Samanlı and Safran Rivers, passing through Yalova Province, were examined in terms of physicochemical parameters, alkalinity and content of inorganic nutrients (nitrate, nitrite, phosphate). The sampling from five station located on the Samanlı and Safran Rivers was performed for fiftythree weeks. Linear and nonlinear Models were applied by the aid of Matlab, Microsoft Excel programs and Multiple Linear Regression Model. The predictions were performed for the samples collected from other points beyond the rivers, and it was shown that the model predicts well the parameters with low error. As a result, it was determined that the linear models predicted the value of parameters for next 8 months. Nonlinear models predict well at least eight months values. Additionally, it was determined that the models work well especially for the samples collected from fresh water.
\end{abstract}

2021 JIENS All rights reserved.

\section{INTRODUCTION}

Water is the most basic need for human health as well as for industrial and agricultural activities. Economic and social achievements of sustainability primarily come from ensuring environmental sustainability. Although three-quarters of our planet is covered with water, few of them are usable. Discharge of agricultural, industrial and municipal wastes is the main source of organic and inorganic pollutants, which accumulate in surface waters, sediments, soil and marine organisms. Many organic and inorganic pollutants, which enter to aquatic environment by discharging, mobilize from pollution source by stream currents, and join into food chain. Because all these pollutants have mutagenic and carcinogenic effects onto endocrine system of organism, the entrance of these pollutions into surface and marine water must be controlled by periodic determination of water quality.

Various statistical techniques have been applied to characterize and to evaluate quality of surface waters. Many researchers have dealt with to develop and to improve the water quality prediction models over the past decade. [1]-[3]. Although the parametric, statistical and deterministic models are traditional approaches for modeling the water quality, they require vast information on various hydrological subprocesses to achieve the result. Most commonly, the water quality monitored by the methods based on the physicochemical parameters [4]. Villas-Boas et al. considered thirteen water quality parameters and identified how some of them were redundant and 
could be removed without significant loss of information. However, obtaining many in situ measurements is both very costly and difficult to implement in many river basins, so some approaches have been developed based on pollutant simulations given by hydrological models [5]. McCaffrey (2012) determined water quality in terms of many parameters by evaluating their effect on each other (such as temperature dependency of dissolved oxygen) [6]. On the other hand, there are many studies on analysis of water quality using linear and nonlinear regression techniques [7][10] . Mustapha and Abdu (2012) evaluated surface water pollution sources in Jakara Basin (Nigeria) by using Principal Component Analysis (PCA). They estimated the contribution of the significant parameters to water quality variation using multiple linear regressions model [11]. Majeed et al. explored the relationship between climate change factors and the Water Quality Index (WQI) of the Euphrates river in Karbala City over the period between 2008 and 2016 using Artificial Neural Network (ANN) and nonlinear multi-regression models [12].

In this study, the water quality was determined as regards $\mathrm{pH}$, conductivity, dissolved oxygen, alkalinity and inorganic nutrients (nitrate, nitrite, phosphate). The analyses were performed for the samples picked from five stations on Samanlı and Safran Rivers. The sampling was carried out every week beyond a year (53 weeks). The results were evaluated by linear and nonlinear equations applied by using Matlab and Microsoft Excel programs and using Multiple Linear Regression Model. Standard deviations belonging the correlations between the experimental data and the theoretical data calculated from the linear and nonlinear models were determined, and their compatibility with each other was examined. Excellent match of the experimental and theoretical values showed that the quality parameters whose analyzes require a long time and/or high cost could be calculated by the aid of these linear and nonlinear models.

\section{EXPERIMENTAL METHOD}

\subsection{Study Area}

Samanll River: It enters the provincial borders of Yalova, named Bedesten River, by feeding from the springs in Taşpınar, Kolaçan and Bedesten Hills in the borders of Gemlik District. It is fed with other rivulets around it. The length of Samanlı River is $22.33 \mathrm{~km}$. It has a water collection area of $81 \mathrm{~km}^{2}$ with an average flow rate of $1.43 \mathrm{~m}^{3} / \mathrm{s}$ and an annual average water volume of 120 million $\mathrm{m}^{3}$.

Safran River: It rises from the northern slopes of Taşpınar and Beşpınar Hills within the borders of Gemlik district and is fed by many rivulets around it. Total length is $23.52 \mathrm{~m}$ and its flow rate is $0.81 \mathrm{~m}^{3} / \mathrm{s}$ [13].

Safran and Samanlı Rivers, which pass through the agricultural fields and the city center, and pour out to Marmara Sea, carry pesticides residues and domestic wastes into the sea (Figure1.). It is foreseen the rivers will become more polluted in this urbanization process, and it is important to determine the extent of the pollution in rivers and examine pollution prevention elements in order to ensure environmental sustainability. 


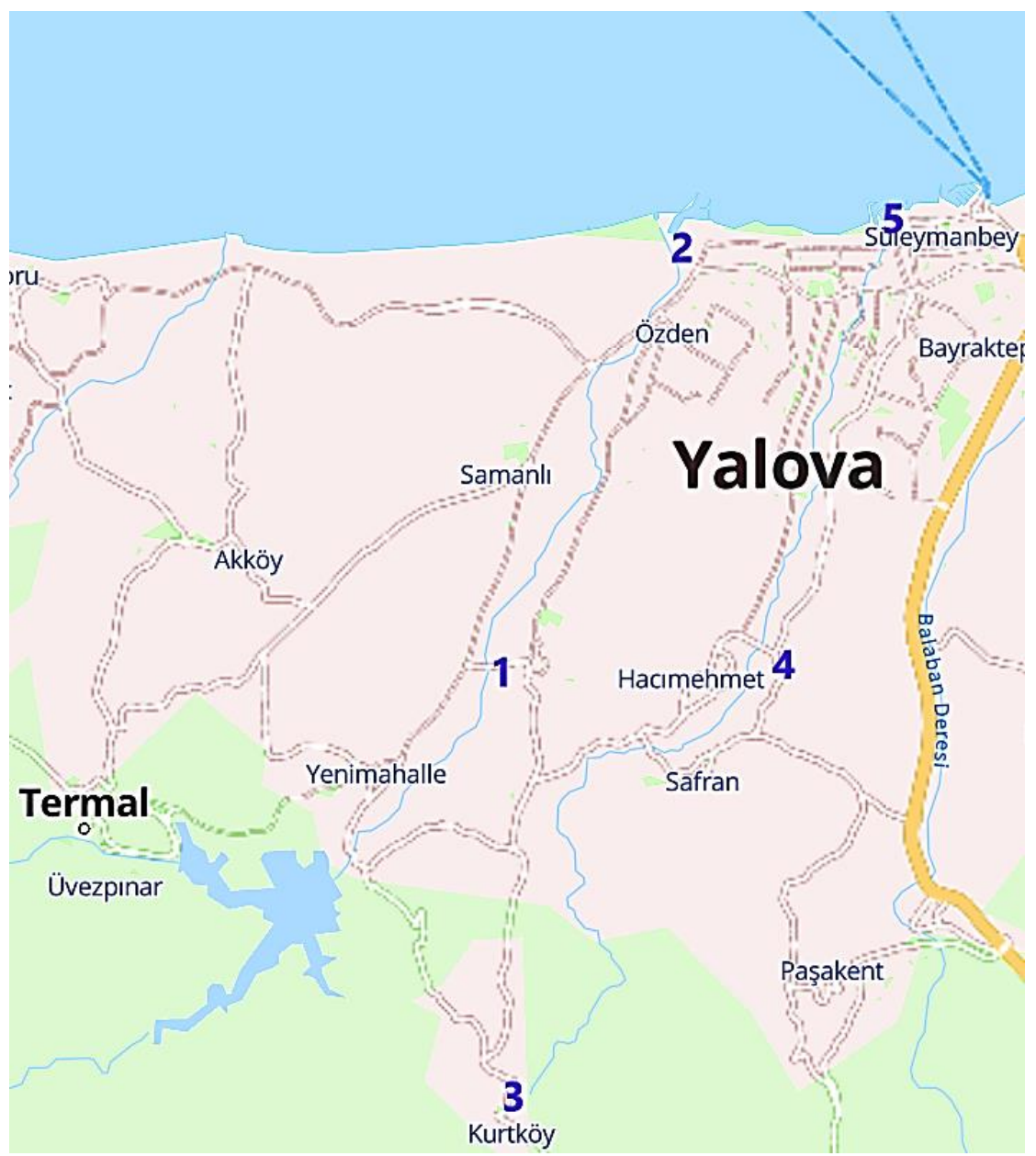

Figure 1. Regions with 5 stations on Samanlı and Safran Rivers

\subsection{Sampling and analysis}

A total of five stations on Samanlı and Safran Rivers were selected for inspection of water quality (Figure 1). The network of sampling stations was designed to cover a wide range of determinants at key sites to reasonably represent the pollution/sewage characteristics of the study area. The water samples were collected from 1 October 2017 to 31 September 2018. The samples were weekly collected from the depth approximately $10 \mathrm{~cm}$ below the surface of the water using bottles. The samples were stored in cooling chests and transported to the laboratory and analyzed within the same day after collection. The determination of quality parameters was according the EPA methods listed in Table 1.

Table 1. Main analysis items and methods

\begin{tabular}{ccc}
\hline Parameter & Analysis Method & Reference \\
\hline $\mathbf{p H}$ & EPA Method, 150.2 & {$[14]$} \\
Electrical Conductivity & EPA Method, 120.1 & {$[15]$} \\
Dissolved Oxygen & EPA Method, 360.1 & {$[16]$} \\
Alkalinity & EPA Method, 310.1 & {$[17]$} \\
Suspended Solids (SS) & EPA Method, 160.2 & {$[18]$} \\
Nitrite \& Nitrate & EPA Method, 353.3 & {$[19]$} \\
Phosphate & EPA Method, 365.3 & {$[20]$} \\
\hline
\end{tabular}


quality

\subsection{Modeling of Experimental Data}

Multiple Linear Regression Method was used to model the data obtained from the experiments. This method has been evaluated in recent years to estimate the efficiency of removing various pollutants from wastewater and environment[21]-[25].

Multiple Linear Regression Analysis is an extension of the simple linear regression method for two or more independent variables. It provides an explanation of the effect and contribution of more than one independent variable for a single continuously dependent variable of a process. The model is produced from experimental data obtained for a complex system [22]. It is generally expressed as equation (1):

$$
y_{i}=\beta_{o}+\beta_{1} x_{i 1}+\beta_{2} x_{i 2}+\cdots \beta_{k} x_{i k}
$$

where $\beta$ is the model coefficients from 0 to $\mathrm{n}$ (it also refers to the contribution of the relevant variable $(x)$ to the process), $y_{i}$ is the estimated dependent variable $(y)$ for any parameter $\mathrm{i}, x_{i}$ denotes different independent $(x)$ variables from 1 to $\mathrm{k}$. In this study, $\mathrm{n}=14$ and $\mathrm{k}$ were 52 . The matrices from which the model equations will be formed are prepared as follows:

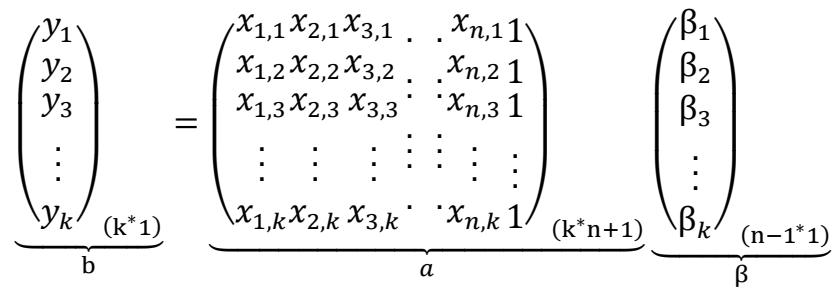

In this way, the model is briefly represented $a \mathrm{~s}=a \times \beta$. Then, using the equation (3), the matrix systems are solved and the coefficients $(\beta)$ to be used in the model equations are calculated.

$$
\beta=a^{-1} \times\left(a^{T}\right)^{-1} \times a^{T} \times b
$$

Nonlinear equations were calculated using the Matlab 2015 program, and the coefficients of determination were calculated using the Matlab 2015 program, using the formula given in equation (4):

$$
R^{2}=\frac{\left(\sum_{i=1}^{n}\left(y_{i}-\bar{y}\right)\left(\hat{y}_{i}-\hat{y}\right)\right)^{2}}{\left(\sum_{i=1}^{n}\left(y_{i}-\bar{y}\right)^{2}\right)\left(\sum_{i=1}^{n}\left(\hat{y}_{i}-\hat{y}\right)^{2}\right)}
$$

The quality parameters corresponding to the $x_{i}$ independent variables used in modeling studies and the quality parameters corresponding to the $y_{i}$ variables were given in Table 2.

Table 2. Quality parameters corresponding to $x_{i}$ and $y_{i}$ independent variables

\begin{tabular}{llll|ll}
\hline $\boldsymbol{x}_{\boldsymbol{i}}$ & Quality Parameter & $\boldsymbol{x}_{\boldsymbol{i}}$ & Quality Parameter & $\boldsymbol{y}_{\boldsymbol{i}}$ & Quality Parameter \\
\hline $\boldsymbol{x}_{\boldsymbol{1}}$ & Air temperature & $x_{9}$ & Phosphate & $y_{1}$ & Dissolved oxygen \\
$\boldsymbol{x}_{\mathbf{2}}$ & Humidity & $x_{10}$ & Nitrite & $y_{2}$ & Electrical conductivity \\
$\boldsymbol{x}_{3}$ & River temperature & $x_{11}$ & Nitrate & $y_{3}$ & $\mathrm{pH}$ \\
$\boldsymbol{x}_{\mathbf{4}}$ & Dissolved oxygen & $x_{12}$ & Air temperature of the & $y_{4}$ & Alkalinity \\
& & & week to be predicted & & \\
$\boldsymbol{x}_{5}$ & Electrical conductivity & $x_{13}$ & Humidity of the week to be & $y_{5}$ & Suspended solid \\
& & & predicted & & \\
$\boldsymbol{x}_{\mathbf{6}}$ & pH & $x_{14}$ & River temperature of the & $y_{6}$ & Phosphate \\
& & week to be predicted & $y_{7}$ & Nitrite \\
$\boldsymbol{x}_{7}$ & Alkalinity & & & $y_{8}$ & Nitrate \\
$\boldsymbol{x}_{8}$ & Suspended solid & & &
\end{tabular}




\section{RESULTS AND DISCUSSIONS}

\subsection{Results of Multiple Linear Regression Analysis}

The equations obtain from application of Multiple Linear Regression Analysis were introduced in Table 3.

Table 3. The model equations evaluated from the experimental results of collected samples from each station

\begin{tabular}{|c|c|c|}
\hline Station & Equation & $\mathbf{R}^{2}$ \\
\hline \multirow{8}{*}{1} & $\begin{array}{c}y 1=0.96 * x 1+21.39 * x 2-1.21 * x 3-0.05 * x 4-0.01 * x 5+3.45 * x 6-0.005 * x 7+0.01 * x 8-1.79 * x 9-8.83 * x 10 \\
+0.03 * x 11+0.11 * x 12+1.86 * x 13-0.22 * x 14-19.58\end{array}$ & 0.283 \\
\hline & $\begin{array}{c}y 2=-1.12 * x 1-55.88 * x 2+4.22 * x 3+1.14 * x 4+0.23 * x 5-2.66 * x 6-0.33 * x 7-0.06 * x 8-122.57 * x 9-131.26 \\
* x 10+2.43 * x 11-9.32 * x 12-78.99 * x 13-0.36 * x 14+573.77\end{array}$ & 0.655 \\
\hline & $\begin{aligned} y 3=-0.009 * x 1- & 0.25 * x 2-0.02 * x 3+0.005 * x 4+0.001 * x 5+0.41 * x 6-0.001 * x 7+0.003 * x 8-0.014 * x 9-0.44 \\
& * x 10-0.008 * x 11-0.035 * x 12-0.503 * x 13+0.073 * x 14+4.99\end{aligned}$ & 0.463 \\
\hline & $\begin{array}{c}y 4=-0.05 * x 1-23.22 * x 2+1.66 * x 3+0.84 * x 4+0.13 * x 5-26.46 * x 6+0.03 * x 7-0.07 * x 8-43.96 * x 9+48 * x 10 \\
-4.63 * x 11-3.64 * x 12-8.42 * x 13-1.56 * x 14+443.62\end{array}$ & 0.663 \\
\hline & $\begin{array}{c}y 5=0.65 * x 1-0.47 * x 2-0.46 * x 3-1.34 * x 4+0.08 * x 5-20.913 * x 6-0.07 * x 7-0.252 * x 8+33.023 * x 9-70.13 \\
* x 10-2.742 * x 11+1.57 * x 12+25.06 * x 3-1.975 * x 14+172.66\end{array}$ & 0.320 \\
\hline & $\begin{array}{c}y 6=0.013 * x 1+0.238 * x 2-.013 * x 3-0.002 * x 4+0.001 * x 5-0.034 * x 6+0.002 * x 7+0.065 * x 9+0.413 * x 10 \\
-0.054 * x 11-0.012 * x 12-0.194 * x 13+0.007 * x 14+0.156\end{array}$ & 0.517 \\
\hline & 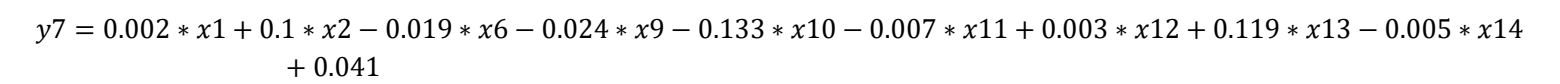 & 0.218 \\
\hline & $\begin{array}{c}y 8=0.066 * x 1+1.673 * x 2+0.031 * x 3-0.004 * x 4+0.006 * x 5-0.42 * x 6-0.012 * x 7-0.008 * x 8-1.605 * x 9-4.949 \\
* x 10+0.124 * x 11-0.09 * x 12+0.044 * x 13-0.097 * x 14+6.618\end{array}$ & 0.396 \\
\hline \multirow{8}{*}{2} & $\begin{array}{c}y 1=0.886 * x 1+17.873 * x 2-1.679 * x 3-0.005 * x 4-0.714 * x 6-0.01 * x 7-0.006 * x 8+4.903 * x 9-19.372 * x 10 \\
-0.037 * x 11-0.003 * x 12+4.67 * x 13+0.659 * x 14+4.498\end{array}$ & 0.410 \\
\hline & $\begin{array}{c}y 2=-241.11 * x 1-7098.40 * x 2+39.00 * x 3-64.18 * x 4+0.17 * x 5-796.49 * x 6+65.04 * x 7-27.11 * x 8-8954.04 * x 9 \\
+48816.27 * x 10-311.97 * x 11-1073.31 * x 12-25125.40 * x 13+876.87 * x 14+35374.40\end{array}$ & 0.235 \\
\hline & $\begin{array}{c}y 3=-0.005 * x 1+0.166 * x 2-0.01 * x 3+0.006 * x 4+0.174 * x 6-0.001 * x 7-0.047 * x 9-0.330 * x 10-0.004 * x 11 \\
-0.021 * x 12-0.162 * x 13+0.029 * x 14+6.677\end{array}$ & 0.283 \\
\hline & $\begin{array}{c}y 4=3.79 * x 1+59.537 * x 2-3.356 * x 3+0.633 * x 4+0.001 * x 5-5.504 * x 6+0.293 * x 7+0.051 * x 8-33.009 * x 9 \\
+96.077 * x 10+0.721 * x 11-3.564 * x 12-33.029 * x 13+2.767 * x 14+174.007\end{array}$ & 0.309 \\
\hline & $\begin{array}{c}y 5=-2.115 * x 1-23.631 * x 2+5.57 * x 3-1.319 * x 4-0.001 * x 5+0.995 * x 6+0.056 * x 7-0.001 * x 8+31.364 * x 9 \\
+37.478 * x 10-1.488 * x 11+2.821 * x 12+49.73 * x 13-6.962 * x 14+27.441\end{array}$ & 0.116 \\
\hline & $\begin{array}{c}y 6=0.007 * x 1+0.134 * x 2-0.007 * x 3+0.002 * x 4+0.028 * x 6+0.001 * x 7+0.21 * x 9-0.327 * x 10-0.002 * x 11 \\
-0.014 * x 12-0.198 * x 13+0.003 * x 14+0.155\end{array}$ & 0.467 \\
\hline & $\begin{aligned} y 7=-0.001 * x 1+ & 0.029 * x 2+0.004 * x 3+0.001 * x 4+0.004 * x 6+0.009 * x 9+0.019 * x 10-0.002 * x 11+0.019 * x 13 \\
& -0.002 * x 14-0.102\end{aligned}$ & 0.370 \\
\hline & $\begin{aligned} y 8=-0.068 * x 1- & 2.845 * x 2-0.289 * x 3-0.234 * x 4+1.289 * x 6-0.011 * x 7-0.006 * x 8-6.506 * x 9-39.572 * x 10 \\
+ & 0.539 * x 11-0.275 * x 12+3.819 * x 13+0.38 * x 14+6.805\end{aligned}$ & 0.563 \\
\hline \multirow{8}{*}{3} & $\begin{array}{c}y 1=0.458 * x 1+11.85 * x 2-0.787 * x 3+0.096 * x 4+0.005 * x 5-4.251 * x 6-0.053 * x 7-0.001 * x 8-3.219 * x 9-5.547 \\
* x 10-3.611 * x 11+0.311 * x 12+3.543 * x 13-x .308 * x 14+66.508\end{array}$ & 0.338 \\
\hline & $\begin{array}{c}y 2=-2.68 * x 1-40.67 * x 2+7.47 * x 3+0.234 * x 4+x 0.36 * x 5+40.64 * x 6+0.066 * x 7+0.102 * x 8-25.133 * x 9 \\
+80.647 * x 10+12.238 * x 11-2.729 * x 12-48.27 * x 13+7.03 * x 14-167.161\end{array}$ & 0.561 \\
\hline & $\begin{array}{c}y 3=-0.013 * x 1-0.075 * x 2+0.055 * x 3+0.006 * x 4+0.001 * x 5+0.337 * x 6-0.003 * x 7-0.007 * x 9+1.731 * x 10 \\
-0.018 * x 11-0.017 * x 12-0.284 * x 13+0.002 * x 14+5.799\end{array}$ & 0.433 \\
\hline & 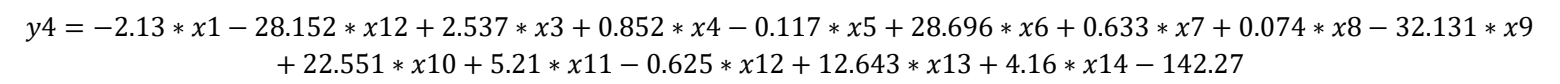 & 0.380 \\
\hline & $\begin{array}{c}y 5=8.25 * x 1+217.46 * x 2-24.895 * x 3-4.82 * x 4+0.63 * x 5-141.712 * x 6-0.44 * x 7-0.138 * x 8-51.98 * x 9 \\
-712.462 * x 10-81.104 * x 11+10.629 * x 12+279.636 * x 13-19.437 * x 14+1156.66\end{array}$ & 0.191 \\
\hline & $\begin{array}{c}y 6=0.001 * x 1+0.137 * x 2-0.009 * x 3+0.006 * x 4-0.009 * x 6+0.001 * x 7+0.022 * x 9-0.883 * x 10-0.157 * x 11 \\
-0.015 * x 12-0.151 * x 13-0.011 * x 14+0.583\end{array}$ & 0.423 \\
\hline & $\begin{array}{c}y 7=0.001 * x 1+0.043 * x 2-0.002 * x 3-0.004 * x 6+0.003 * x 9-0.141 * x 10-0.003 * x 11+0.002 * x 12+0.055 * 13 \\
-0.003 * x 14-0.069\end{array}$ & 0.220 \\
\hline & $\begin{array}{c}y 8=0.012 * x 1+0.528 * x 2+0.003 * x 3+0.008 * x 4+0.002 * x 5+0.005 * x 6-0.004 * x 7-0.55 * x 9+1.248 * x 10+0.569 \\
* x 11-0.013 * x 12+0.209 * x 13-0.019 * x 14+0.477\end{array}$ & 0.607 \\
\hline \multirow[b]{2}{*}{4} & $\begin{array}{c}y 1=0.331 * x 1+7.876 * x 2-0.429 * x 3+0.034 * x 4+0.003 * x 5-0.176 * x 6+0.011 * x 7+0.003 * x 8+0.5 * x 9-25.612 \\
* x 10-0.332 * x 11+0.531 * x 12+7.133 * x 13-0.867 * x 14+3.055\end{array}$ & 0.221 \\
\hline & $\begin{array}{c}-5.19 * x 1-93.88 * x 2+7.964 * x 3-1.004 * x 4+0.51 * x 5+16.816 * x 6+0.425 * x 7+0.048 * x 8-41.439 * x 9 \\
-25.014 * x 10+4.435 * x 11-7.829 * x 12-125.448 * x 13+10.419 * x 14+98.229\end{array}$ & 0.744 \\
\hline
\end{tabular}


quality

$$
\begin{gathered}
y 3=-0.019 * x 1-0.23 * x 2+0.031 * x 3+0.004 * x 4+0.498 * x 6-0.209 * x 9+1.16 * x 10+0.015 * x 11-0.009 * x 12 \\
-0.078 * x 13-0.004 * x 14+4.403
\end{gathered}
$$

$y 4=0.927 * x 1+0.787 * x 2-4.094 * x 3 * 1.18 * x 4+0.132 * x 5+16.36 * x 6+0.324 * x 7-0.002 * x 8+1.131 * x 9+69.418$

$$
* x 10+2.862 * x 11+0.036 * x 12-17.702 * x 13+2.447 * x 14+18.623
$$

$y 5=42.98 * x 1+828.35 * x 2-59.6 * x 3-14.015 * x 4+1.642 * x 5-499.86 * x 6-3.78 * x 7-0.29 * x 8+152.75 * x 9$ $-641.23 * x 10-26.06 * x 11+34.75 * x 12+741.29 * x 13-46.285 * x 14+3711.51$

$y 6=0.005 * x 1+0.123 * x 2-0.013 * x 3-0.006 * x 4+0.001 * x 5-0.279 * x 6+0.095 * x 9+0.22 * x 10-0.099 * x 11$

$$
-0.018 * x 12-0.29 * x 13+0.013 * x 14+2.84
$$

$y 7=-0.003 * x 1-0.005 * x 2+0.006 * x 3+0.001 * x 4+0.043 * x 6+0.004 * x 9-0.165 * x 10-0.003 * x 12-0.013 * x 13$ $+0.009 * x 14-0.414$

$y 8=0.044 * x 1+0.203 * x 2-0.022 * x 3-0.021 * x 4+0.004 * x 5-0.827 * x 6-0.004 * x 7-0.001 * x 8-0.908 * x 9+1.21$ $* x 10+0.483 * x 11-0.053 * x 12-0.548 * x 13+0.034 * x 14+7.794$

$y 1=0.611 * x 1+7.793 * x 2-0.836 * x 3-0.074 * x 4+5.682 * x 6-0.038 * x 7+0.001 * x 8+5.919 * x 9+16.265 * x 10$ $-0.67 * x 11+0.314 * x 12+1.716 * x 13-0.296 * x 14-33.803$

$y 2=-1202.47 * x 1-22941.72 * x 2+1193.53 * x 3-309.05 * x 4+0.155 * x 5-17824.18 * x 6+46.86 * x 7+19.07 * x 8$ $-16700.59 * x 9-32348.43 * x 10-1414.29 * x 11+911.74 * x 12+9358.57 * x 13-456.45 * x 14$ $+162063.48$

$y 3=0.011 * x 1+0.1 * x 2-0.023 * x 3+0.005 * x 4+0.233 * x 6+0.001 * x 8+0.146 * x 9-0.07 * x 10-0.03 * x 11-0.021$ $* x 12-0.276 * x 13+0.033 * x 14+6.244$

$$
\begin{array}{r}
y 4=1.59 * x 1+51.34 * x 2-1.327 * x 3-0.467 * x 4+0.001 * x 5+29.535 * x 6-0.104 * x 7-0.103 * x 8+18.065 * x 9 \\
+114.915 * x 10+12.906 * x 11-3.112 * x 12-36.543 * x 13+0.412 * x 14+45.85
\end{array}
$$

$y 5=-2.23 * x 1+11.47 * x 2+3.05 * x 3-3.80 * x 4-0.001 * x 5-27.343 * x 6+0.08 * x 7+0.049 * x 8+63.01 * x 9$ $-164.303 * x 10-17.827 * x 11+4.774 * x 12+132.366 * x 13-6.339 * x 14+208.909$

$y 6=0.003 * x 1+0.092 * x 2-0.005 * x 3+0.003 * x 4-0.014 * x 6+0.002 * x 7+0.001 * x 8+0.086 * x 9-0.618 * x 10$ $-0.031 * x 11-0.012 * x 12-0.229 * x 13+0.006 * x 14+0.298$

$y 7=-0.001 * x 1+0.093 * x 2+0.002 * x 3-0.001 * x 4-0.052 * x 6+0.032 * x 9-0.063 * x 10-0.005 * x 11-0.003 * x 12$ $+0.014 * x 13+0.001 * x 14+0.41$

$y 8=0.03 * x 1-0.134 * x 2+0.049 * x 3+0.006 * x 4+0.142 * x 6-0.001 * x 7+0.021 * x 9+2.586 * x 10+0.294 * x 11$ $-0.095 * x 12-1.733 * x 13-0.019 * x 14+1.434$

\begin{tabular}{|c|c|c|c|c|c|c|c|c|c|c|}
\hline & & & $\mathrm{y}_{1}$ & $\mathrm{y}_{2}$ & $\mathrm{y}_{3}$ & $\mathrm{y}_{4}$ & $\mathrm{y}_{5}$ & $\mathrm{y}_{6}$ & $\mathrm{y}_{7}$ & $\mathrm{y}_{8}$ \\
\hline & $\mathrm{x}_{1}$ & & $8.88 \mathrm{E}-02$ & $1.48 \mathrm{E}+00$ & $5.73 \mathrm{E}-03$ & 9.72E-01 & $7.28 \mathrm{E}-01$ & $2.13 \mathrm{E}-03$ & $-2.45 \mathrm{E}-04$ & $2.01 \mathrm{E}-02$ \\
\hline & $\mathrm{x}_{2}$ & & $2.42 \mathrm{E}-02$ & $1.95 \mathrm{E}-01$ & $-3.86 \mathrm{E}-04$ & 6.79E-02 & $1.23 \mathrm{E}-01$ & $1.68 \mathrm{E}-04$ & $-2.21 \mathrm{E}-04$ & $2.45 \mathrm{E}-03$ \\
\hline & $\mathrm{x}_{3}$ & & $5.64 \mathrm{E}-02$ & $1.12 \mathrm{E}+00$ & $4.54 \mathrm{E}-03$ & 7.53E-01 & $6.08 \mathrm{E}-01$ & $1.28 \mathrm{E}-03$ & $-3.04 \mathrm{E}-04$ & $1.35 \mathrm{E}-02$ \\
\hline & $\mathrm{x}_{4}$ & & $-4.45 \mathrm{E}-02$ & $-6.88 \mathrm{E}-01$ & $8.98 \mathrm{E}-04$ & $-3.89 \mathrm{E}-01$ & $-3.14 \mathrm{E}-01$ & $-1.90 \mathrm{E}-03$ & $2.20 \mathrm{E}-04$ & $-1.32 \mathrm{E}-02$ \\
\hline & $\mathrm{x}_{5}$ & & $-1.14 \mathrm{E}-01$ & $1.41 \mathrm{E}-01$ & $2.26 \mathrm{E}-02$ & $7.60 \mathrm{E}-01$ & $1.31 \mathrm{E}+00$ & $1.05 \mathrm{E}-03$ & $1.25 \mathrm{E}-03$ & $2.81 \mathrm{E}-02$ \\
\hline & $\mathrm{x}_{6}$ & & $1.24 \mathrm{E}-01$ & $1.04 \mathrm{E}+00$ & $-7.52 \mathrm{E}-04$ & $3.46 \mathrm{E}-01$ & 4.34E-01 & $7.90 \mathrm{E}-04$ & $-7.20 \mathrm{E}-04$ & $1.46 \mathrm{E}-02$ \\
\hline & $\mathrm{x}_{7}$ & & $-1.69 \mathrm{E}-01$ & $-4.64 \mathrm{E}-01$ & $2.69 \mathrm{E}-02$ & $8.60 \mathrm{E}-01$ & $-1.07 \mathrm{E}-01$ & $2.37 \mathrm{E}-03$ & $3.95 \mathrm{E}-03$ & 5.39E-03 \\
\hline & $\mathrm{X}_{8}$ & & $-6.87 \mathrm{E}-02$ & $-3.93 \mathrm{E}-01$ & $3.87 \mathrm{E}-03$ & $-2.15 \mathrm{E}-02$ & $-2.42 \mathrm{E}-01$ & 7.39E-04 & $5.18 \mathrm{E}-04$ & $-6.91 \mathrm{E}-03$ \\
\hline & $\mathrm{X}_{9}$ & & $1.36 \mathrm{E}-02$ & $1.25 \mathrm{E}-01$ & $2.05 \mathrm{E}-04$ & $6.99 \mathrm{E}-02$ & $5.64 \mathrm{E}-02$ & $3.02 \mathrm{E}-04$ & $-6.06 \mathrm{E}-05$ & $1.84 \mathrm{E}-03$ \\
\hline & $\mathrm{x}_{10}$ & & $5.44 \mathrm{E}-04$ & $1.19 \mathrm{E}-02$ & $1.56 \mathrm{E}-04$ & $1.77 \mathrm{E}-02$ & $1.25 \mathrm{E}-02$ & $6.74 \mathrm{E}-05$ & $-1.03 \mathrm{E}-05$ & $2.08 \mathrm{E}-07$ \\
\hline & $\mathrm{x}_{11}$ & & $9.47 \mathrm{E}-03$ & $2.88 \mathrm{E}-02$ & $-2.23 \mathrm{E}-04$ & $-4.78 \mathrm{E}-03$ & $-3.15 \mathrm{E}-02$ & $1.15 \mathrm{E}-04$ & $1.23 \mathrm{E}-04$ & $9.00 \mathrm{E}-04$ \\
\hline & $\mathrm{x}_{12}$ & & $1.24 \mathrm{E}-02$ & $7.40 \mathrm{E}-01$ & $1.06 \mathrm{E}-02$ & $1.11 \mathrm{E}+00$ & $9.40 \mathrm{E}-01$ & $3.21 \mathrm{E}-03$ & $9.82 \mathrm{E}-05$ & $1.68 \mathrm{E}-03$ \\
\hline & $\mathrm{x}_{13}$ & & $9.65 \mathrm{E}-03$ & $4.55 \mathrm{E}-02$ & $3.01 \mathrm{E}-04$ & $-3.01 \mathrm{E}-03$ & $-3.02 \mathrm{E}-02$ & $5.71 \mathrm{E}-05$ & $2.87 \mathrm{E}-05$ & $6.97 \mathrm{E}-04$ \\
\hline & $\mathrm{x}_{14}$ & & $1.30 \mathrm{E}-01$ & $1.31 \mathrm{E}+00$ & $3.45 \mathrm{E}-03$ & $6.87 \mathrm{E}-01$ & 8.19E-01 & $1.10 \mathrm{E}-03$ & $-1.08 \mathrm{E}-03$ & $1.29 \mathrm{E}-02$ \\
\hline $\mathrm{x}_{1}$ & * & $\mathrm{x}_{1}$ & $2.18 \mathrm{E}-01$ & $8.28 \mathrm{E}-01$ & $4.62 \mathrm{E}-03$ & $3.24 \mathrm{E}-01$ & $4.51 \mathrm{E}-01$ & $-3.03 \mathrm{E}-03$ & $-5.73 \mathrm{E}-03$ & $-1.05 \mathrm{E}-02$ \\
\hline $\mathrm{x}_{2}$ & $*$ & $\mathrm{x}_{2}$ & $1.43 \mathrm{E}-02$ & $1.54 \mathrm{E}-01$ & $-7.17 \mathrm{E}-04$ & $9.55 \mathrm{E}-02$ & $2.04 \mathrm{E}-01$ & $1.69 \mathrm{E}-04$ & $-3.41 \mathrm{E}-04$ & $9.77 \mathrm{E}-04$ \\
\hline
\end{tabular}

\subsection{Results of Nonlinear Models}

The $x$ and $y$ variables, their coefficient and determination coefficient of equations belonging non-linear models were presented in Tables 4-8.

Table 4. The $\mathrm{x}$ and $\mathrm{y}$ variables, their coefficient and determination coefficient of equations belonging non-linear models yielded from the data collected Station 1. 


\begin{tabular}{|c|c|c|c|c|c|c|c|c|c|c|}
\hline & * & $\mathrm{x}_{3}$ & 01 & +00 & 2 & 0 & $9.16 \mathrm{E}-01$ & 3 & $-5.12 \mathrm{E}-03$ & 34 \\
\hline $\mathrm{x}_{4}$ & $*$ & $\mathrm{x}_{4}$ & 4.86E-02 & $-8.66 \mathrm{E}-01$ & $2.06 \mathrm{E}-03$ & $-3.43 \mathrm{E}-01$ & $-4.24 \mathrm{E}-02$ & $1.48 \mathrm{E}-03$ & $2.85 \mathrm{E}-03$ & $-1.48 \mathrm{E}-02$ \\
\hline$x_{5}$ & * & $\mathrm{x}_{5}$ & $44 \mathrm{E} 02$ & 89E-02 & 02505 & $1.21 \mathrm{E}-02$ & $101 \mathrm{E} 02$ & $.91 \mathrm{E}-06$ & 432505 & $3.45 \mathrm{E}-\mathrm{C}$ \\
\hline 6 & * & $\mathrm{x}_{6}$ & $.08 \mathrm{E}+00$ & $8.26 \mathrm{E}+00$ & $1.94 \mathrm{E}-02$ & $2.07 \mathrm{E}+00$ & $10 \mathrm{E}+00$ & $5.29 \mathrm{E}-03$ & $-6.77 \mathrm{E}-03$ & $1.16 \mathrm{E}-01$ \\
\hline$x_{7}$ & * & $x_{7}$ & $495 \mathrm{~F}_{0} 03$ & E-02 & OOF 04 & $2.60 \mathrm{E}-02$ & 4 45E-03 & $53 \mathrm{E}-05$ & $1.16 \mathrm{E}-04$ & $38 \mathrm{E}-04$ \\
\hline $\mathrm{x}_{8}$ & * & $\mathrm{x}_{8}$ & $.22 \mathrm{E}-03$ & $4.90 \mathrm{E}-02$ & $-2.59 \mathrm{E}-04$ & $5.58 \mathrm{E}-03$ & $6.59 \mathrm{E}-02$ & $2.14 \mathrm{E}-04$ & $-3.27 \mathrm{E}-04$ & $6.12 \mathrm{E}-05$ \\
\hline $\mathrm{x}_{9}$ & $*$ & $\mathrm{X}_{9}$ & $15 \mathrm{E}-03$ & 4.30 & $80 \mathrm{E}-04$ & 2 & 1.011 & $.23 \mathrm{E}-04$ & $5.51 \mathrm{I}$ & $9.20 \mathrm{E}-04$ \\
\hline $\mathrm{x}_{10}$ & $*$ & $\mathrm{x}_{10}$ & $13 \mathrm{E}-03$ & $5.80 \mathrm{E}-06$ & $5.48 \mathrm{E}-05$ & $-7.52 \mathrm{E}-03$ & $-2.00 \mathrm{E}-02$ & $1.77 \mathrm{E}-06$ & $4.38 \mathrm{E}-05$ & 2.07E-04 \\
\hline $\mathrm{x}_{11}$ & * & $\mathrm{x}_{11}$ & $84 \mathrm{E}-$ & (50 & 3 & 1 & 1.90 & 3 & 4. & $6 \mathrm{E}-02$ \\
\hline $\mathrm{x}_{12}$ & $*$ & $\mathrm{x}_{12}$ & 3.01E-01 & $2.74 \mathrm{E}+00$ & $-3.76 \mathrm{E}-03$ & $6.18 \mathrm{E}-01$ & $8.22 \mathrm{E}-01$ & $5.90 \mathrm{E}-03$ & $-2.62 \mathrm{E}-03$ & $5.95 \mathrm{E}-02$ \\
\hline $\mathrm{x}_{13}$ & * & $\mathrm{x}_{13}$ & $52 \mathrm{E}-02$ & E-01 & 4 & 1 & 2 & 94 & 4.7 & $81 \mathrm{E}-03$ \\
\hline $\mathrm{x}_{14}$ & $*$ & $\mathrm{x}_{14}$ & $.41 \mathrm{E}-01$ & $1.38 \mathrm{E}+00$ & $-9.79 \mathrm{E}-03$ & $-3.23 \mathrm{E}-01$ & $1.64 \mathrm{E}-01$ & $1.28 \mathrm{E}-02$ & $-1.83 \mathrm{E}-03$ & $.08 \mathrm{E}-02$ \\
\hline $\mathrm{x}_{1}$ & $*$ & $\mathrm{x}_{2}$ & 83E-01 & $3.19 \mathrm{E}+00$ & $-1.21 \mathrm{E}-02$ & $1.57 \mathrm{E}+00$ & $4.28 \mathrm{E}+00$ & 4.65E-03 & $-6.71 \mathrm{E}-03$ & 3.73E-02 \\
\hline $\mathrm{x}_{1}$ & $*$ & $\mathrm{x}_{3}$ & $-4.57 \mathrm{E}-01$ & $1.45 \mathrm{E}+00$ & $-3.61 \mathrm{E}-02$ & $-1.57 \mathrm{E}+00$ & $-6.78 \mathrm{E}-01$ & $5.79 \mathrm{E}-03$ & $5.23 \mathrm{E}-03$ & $6.44 \mathrm{E}-02$ \\
\hline $\mathrm{x}_{1}$ & $*$ & $\mathrm{x}_{4}$ & $-2.91 \mathrm{E}-02$ & $-7.88 \mathrm{E}-01$ & $-1.80 \mathrm{E}-03$ & $-4.78 \mathrm{E}-01$ & $-9.08 \mathrm{E}-01$ & 8.61E-04 & $1.68 \mathrm{E}-03$ & $1.83 \mathrm{E}-03$ \\
\hline $\mathrm{x}_{1}$ & $*$ & $\mathrm{x}_{5}$ & $28 \mathrm{E}-02$ & $1.96 \mathrm{E}-01$ & $1.41 \mathrm{E}-05$ & $-7.18 \mathrm{E}-02$ & $5.34 \mathrm{E}-02$ & $-5.27 \mathrm{E}-05$ & $-4.80 \mathrm{E}-04$ & $-2.01 \mathrm{E}-03$ \\
\hline $\mathrm{x}_{1}$ & $*$ & $\mathrm{x}_{6}$ & $-2.77 \mathrm{E}-01$ & $-1.51 \mathrm{E}+00$ & $1.36 \mathrm{E}-03$ & $.00 \mathrm{E}+00$ & $-a^{2}$ & 4 & 7. & E-02 \\
\hline $\mathrm{x}_{1}$ & * & $\mathrm{x}_{7}$ & $1.29 \mathrm{E}-02$ & $.13 \mathrm{E}-01$ & $3.89 \mathrm{E}-04$ & $2.19 \mathrm{E}-01$ & 4.99E-03 & $.31 \mathrm{E}-05$ & $9.75 \mathrm{E}-04$ & $.69 \mathrm{E}-03$ \\
\hline $\mathrm{x}_{1}$ & $*$ & $\mathrm{x}_{8}$ & $-2.13 \mathrm{E}-02$ & $-2.13 \mathrm{E}-01$ & $-1.05 \mathrm{E}-03$ & $-2.81 \mathrm{E}-01$ & $-3.55 \mathrm{E}-01$ & $-1.82 \mathrm{E}-03$ & $1.10 \mathrm{E}-03$ & $-5.68 \mathrm{E}-03$ \\
\hline $\mathrm{x}_{1}$ & $*$ & $\mathrm{X}_{9}$ & 3.66E-01 & $4.04 \mathrm{E}+00$ & $1.50 \mathrm{E}-02$ & $3.00 \mathrm{E}+00$ & $.41 \mathrm{E}+00$ & $1.28 \mathrm{E}-02$ & $-2.47 \mathrm{E}-03$ & $6.09 \mathrm{E}-02$ \\
\hline $\mathrm{x}_{1}$ & $*$ & $\mathrm{x}_{10}$ & 13E-01 & A1F 01 & $8.18 \mathrm{E}-04$ & 3.07E-01 & 5.4 & -04 & -1 & E-03 \\
\hline $\mathrm{x}_{1}$ & $*$ & $\mathrm{x}_{11}$ & $-2.31 \mathrm{E}-01$ & 4.27E+00 & $1.42 \mathrm{E}-02$ & $1.76 \mathrm{E}+00$ & $3.42 \mathrm{E}+00$ & $-8.54 \mathrm{E}-04$ & $7.04 \mathrm{E}-03$ & $-5.37 \mathrm{E}-02$ \\
\hline $\mathrm{x}_{1}$ & $*$ & $\mathrm{x}_{12}$ & $3.82 \mathrm{E}-02$ & $.07 \mathrm{E}+00$ & $1.46 \mathrm{E}-02$ & $1.32 \mathrm{E}+00$ & -1.1 & $2 \mathrm{E}-03$ & $3.64 \mathrm{E}-04$ & $3.16 \mathrm{E}-02$ \\
\hline $\mathrm{x}_{1}$ & * & $\mathrm{x}_{13}$ & $-5.94 \mathrm{E}-01$ & $5.07 \mathrm{E}+00$ & $3.21 \mathrm{E}-02$ & $-2.23 \mathrm{E}+00$ & $-4.74 \mathrm{E}+00$ & $-8.78 \mathrm{E}-04$ & $9.40 \mathrm{E}-03$ & $-8.67 \mathrm{E}-02$ \\
\hline $\mathrm{x}_{1}$ & $*$ & $\mathrm{x}_{14}$ & 2.19E-01 & $6.91 \mathrm{E}-01$ & $28 \mathrm{E}-03$ & $5.20 \mathrm{E}-02$ & $1.94 \mathrm{E}+00$ & $2.36 \mathrm{E}-03$ & $-4.15 \mathrm{E}-04$ & $2.52 \mathrm{E}-02$ \\
\hline $\mathrm{x}_{2}$ & * & $\mathrm{x}_{3}$ & $2.04 \mathrm{E}-01$ & $32 \mathrm{E}+00$ & $-2.95 \mathrm{E}-04$ & $1.59 \mathrm{E}+00$ & $3.14 \mathrm{E}+00$ & $3.28 \mathrm{E}-03$ & -4.7 & $.40 \mathrm{E}-02$ \\
\hline $\mathrm{x}_{2}$ & $*$ & $\mathrm{x}_{4}$ & 91E-02 & 2.37E-01 & $-6.35 \mathrm{E}-03$ & $2.68 \mathrm{E}-01$ & $1.97 \mathrm{E}+00$ & 1.69E-03 & $-4.11 \mathrm{E}-03$ & $-5.93 \mathrm{E}-03$ \\
\hline $\mathrm{x}_{2}$ & * & $\mathrm{x}_{5}$ & $-5.74 \mathrm{E}-01$ & $5.31 \mathrm{E}+00$ & $-8.70 \mathrm{E}-03$ & $-2.77 \mathrm{E}+00$ & $-1.58 \mathrm{E}+00$ & $-7.29 \mathrm{E}-03$ & -6.0 & $-5.36 \mathrm{E}-02$ \\
\hline $\mathrm{x}_{2}$ & $*$ & $\mathrm{x}_{6}$ & 32E-01 & $86 \mathrm{E}+00$ & $-5.03 \mathrm{E}-03$ & $5.83 \mathrm{E}-01$ & $1.10 \mathrm{E}+00$ & $1.31 \mathrm{E}-03$ & $-2.22 \mathrm{E}-03$ & $2.35 \mathrm{E}-02$ \\
\hline & $*$ & $x_{7}$ & $16 \mathrm{~F}+00$ & $04 \mathrm{~F}_{+} \mathrm{C}$ & $1.36 \mathrm{E}-02$ & $5.13 \mathrm{E}+00$ & $2.13 \mathrm{E}+00$ & $\mathrm{E}-02$ & -03 & $1.22 \mathrm{E}-01$ \\
\hline $\mathrm{x}_{2}$ & $*$ & $\mathrm{x}_{8}$ & $-6.83 \mathrm{E}-01$ & $4.99 \mathrm{E}+00$ & $-1.39 \mathrm{E}-03$ & $-1.78 \mathrm{E}+00$ & $-8.20 \mathrm{E}-01$ & $-4.80 \mathrm{E}-03$ & $-9.41 \mathrm{E}-06$ & $-7.36 \mathrm{E}-02$ \\
\hline $\mathrm{X}_{2}$ & $*$ & $\mathrm{x}_{9}$ & 05500 & ( 0 ( & 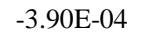 & & & & -6 & \\
\hline $\mathrm{x}_{2}$ & $*$ & $\mathrm{x}_{10}$ & $1.47 \mathrm{E}-03$ & $1.23 \mathrm{E}-02$ & $-1.65 \mathrm{E}-04$ & $-3.07 \mathrm{E}-02$ & $-3.01 \mathrm{E}-02$ & $-1.73 \mathrm{E}-04$ & $1.64 \mathrm{E}-05$ & 2.72E-04 \\
\hline $\mathrm{x}_{2}$ & * & $\mathrm{x}_{11}$ & $-3.89 \mathrm{E}-02$ & $-4.63 \mathrm{E}-01$ & $-2.20 \mathrm{E}-0 \mathrm{~J}$ & $-4.09 \mathrm{E}-01$ & $-4.22 \mathrm{E}-01$ & 3 & 4 & $-4.52 \mathrm{E}-03$ \\
\hline $\mathrm{x}_{2}$ & * & $\mathrm{x}_{12}$ & $62 \mathrm{E}-01$ & $12 \mathrm{E}+00$ & $3.14 \mathrm{E}-03$ & $9.67 \mathrm{E}-01$ & $3.11 \mathrm{E}+00$ & 4.17E-03 & $-4.55 \mathrm{E}-03$ & $1.11 \mathrm{E}-03$ \\
\hline $\mathrm{x}_{2}$ & $*$ & $\mathrm{x}_{13}$ & $2.67 \mathrm{E}-02$ & 1 & $-7.65 \mathrm{E}-04$ & 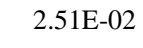 & $4.76 \mathrm{E}-02$ & $2.85 \mathrm{E}-05$ & -9 & $3.07 \mathrm{E}-0$ \\
\hline $\mathrm{x}_{2}$ & * & $\mathrm{x}_{14}$ & $.75 \mathrm{E}-02$ & $3.10 \mathrm{E}-01$ & $-5.23 \mathrm{E}-03$ & 4.77E-02 & $1.93 \mathrm{E}+00$ & $.35 \mathrm{E}-04$ & $-3.57 \mathrm{E}-03$ & $-6.39 \mathrm{E}-03$ \\
\hline$x_{3}$ & * & $\mathrm{x}_{4}$ & . & הת חקרט & 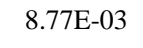 & - & $\pi x^{\circ}$ & 2 & -3 & $1.42 \mathrm{E}-02$ \\
\hline $\mathrm{x}_{3}$ & * & $\mathrm{x}_{5}$ & 87 & -01 & 1.41 & $-9.89 \mathrm{E}-02$ & -2 & -04 & 1.1 & $-8.59 \mathrm{E}-03$ \\
\hline$x_{3}$ & * & $\mathrm{x}_{6}$ & 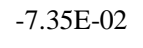 & $x^{2}+2$ & 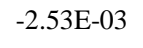 & $2.30 \mathrm{E}-01$ & -2 & 4 & $1.44 \mathrm{E}-03$ & $2.31 \mathrm{E}-02$ \\
\hline $\mathrm{x}_{3}$ & $*$ & $\mathrm{x}_{7}$ & $15 \mathrm{E}-02$ & $5.41 \mathrm{E}-03$ & $-3.46 \mathrm{E}-03$ & 4 & 1 & $E-04$ & $-1.81 \mathrm{E}-03$ & $\mathrm{E}-02$ \\
\hline$x_{3}$ & * & $\mathrm{x}_{8}$ & 300 & $\mathrm{E}-01$ & $6.29 \mathrm{E}-04$ & -2.7 & 4.84E-02 & 94 & 04 & $4 \mathrm{E}-\mathrm{U}$ \\
\hline $\mathrm{A}_{3}$ & 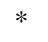 & $\mathrm{X}_{9}$ & $4.68 \mathrm{E}-01$ & $4.39 \mathrm{E}+00$ & $2.58 \mathrm{E}-03$ & $2.23 \mathrm{E}+00$ & $2.55 \mathrm{E}+00$ & $7.88 \mathrm{E}-03$ & $-4.52 \mathrm{E}-03$ & $6.22 \mathrm{E}-02$ \\
\hline$x_{3}$ & * & $\mathrm{x}_{10}$ & 00E-02 & $4.68 \mathrm{E}-01$ & $-2.13 \mathrm{E}-03$ & $6.12 \mathrm{E}-02$ & 4.37E-01 & $-7.63 \mathrm{E}-04$ & $-1.39 \mathrm{E}-03$ & $3.01 \mathrm{E}-$ \\
\hline & $*$ & $\mathrm{x}_{11}$ & $-2.15 \mathrm{E}-01$ & $-3.12 \mathrm{E}+00$ & $1.54 \mathrm{E}-02$ & $-4.83 \mathrm{E}-01$ & $-8.71 \mathrm{E}-01$ & $3.25 \mathrm{E}-03$ & $2.71 \mathrm{E}-03$ & $-6.25 \mathrm{E}-02$ \\
\hline
\end{tabular}


quality

\begin{tabular}{|c|c|c|c|c|c|c|c|c|c|c|}
\hline$x_{3}$ & $*$ & $\mathrm{x}_{12}$ & $2.24 \mathrm{E}-01$ & $-3.68 \mathrm{E}+00$ & $-2.04 \mathrm{E}-02$ & $-3.15 \mathrm{E}+00$ & $-1.98 \mathrm{E}+00$ & $-8.43 \mathrm{E}-03$ & $2.72 \mathrm{E}-03$ & $-2.65 \mathrm{E}-02$ \\
\hline$x_{3}$ & $*$ & $\mathrm{x}_{13}$ & $-5.47 \mathrm{E}-01$ & $-4.89 \mathrm{E}+00$ & $1.34 \mathrm{E}-02$ & $-2.33 \mathrm{E}+00$ & $-3.31 E+00$ & $-4.47 \mathrm{E}-03$ & 5.19E-03 & $-6.46 \mathrm{E}-02$ \\
\hline$x_{3}$ & $*$ & $\mathrm{x}_{14}$ & $-4.79 \mathrm{E}-01$ & $6.75 \mathrm{E}-01$ & $7.62 \mathrm{E}-03$ & $1.40 \mathrm{E}+00$ & $-6.25 \mathrm{E}-01$ & $-1.24 \mathrm{E}-02$ & $2.10 \mathrm{E}-03$ & $-4.89 \mathrm{E}-02$ \\
\hline $\mathrm{x}_{4}$ & $*$ & $\mathrm{x}_{5}$ & $-5.26 \mathrm{E}-03$ & $1.50 \mathrm{E}-02$ & $-9.77 \mathrm{E}-04$ & $-5.53 \mathrm{E}-02$ & $-9.65 \mathrm{E}-02$ & $6.60 \mathrm{E}-05$ & $2.03 \mathrm{E}-04$ & $2.84 \mathrm{E}-04$ \\
\hline $\mathrm{x}_{4}$ & $*$ & $\mathrm{x}_{6}$ & $1.33 \mathrm{E}-01$ & $-9.38 \mathrm{E}-01$ & $-2.94 \mathrm{E}-02$ & $-1.80 \mathrm{E}+00$ & $1.22 \mathrm{E}+00$ & $-1.20 \mathrm{E}-02$ & $-8.09 \mathrm{E}-03$ & $-6.03 \mathrm{E}-02$ \\
\hline $\mathrm{X}_{4}$ & $*$ & $\mathrm{x}_{7}$ & $1.31 \mathrm{E}-02$ & $1.02 \mathrm{E}-01$ & $2.38 \mathrm{E}-03$ & $2.05 \mathrm{E}-01$ & $1.43 \mathrm{E}-01$ & $2.36 \mathrm{E}-04$ & $-3.75 \mathrm{E}-04$ & $2.96 \mathrm{E}-03$ \\
\hline $\mathrm{X}_{4}$ & $*$ & $\mathrm{x}_{8}$ & $-4.24 \mathrm{E}-02$ & $-6.65 \mathrm{E}-01$ & $6.29 \mathrm{E}-04$ & $-2.79 \mathrm{E}-01$ & $4.84 \mathrm{E}-02$ & $5.91 \mathrm{E}-04$ & $8.23 \mathrm{E}-04$ & $-1.24 \mathrm{E}-02$ \\
\hline $\mathrm{X}_{4}$ & $*$ & $\mathrm{X}_{9}$ & $-2.73 \mathrm{E}-01$ & $-2.18 \mathrm{E}+00$ & $9.68 \mathrm{E}-03$ & $-2.07 \mathrm{E}-01$ & $-3.89 \mathrm{E}-01$ & $2.95 \mathrm{E}-03$ & $1.07 \mathrm{E}-03$ & $-2.97 \mathrm{E}-02$ \\
\hline $\mathrm{x}_{4}$ & * & $\mathrm{x}_{10}$ & $-1.32 \mathrm{E}-02$ & $-1.69 \mathrm{E}-01$ & $2.90 \mathrm{E}-03$ & $6.69 \mathrm{E}-02$ & $-1.96 \mathrm{E}-01$ & $1.24 \mathrm{E}-03$ & $5.01 \mathrm{E}-04$ & $-1.92 \mathrm{E}-03$ \\
\hline$x_{4}$ & $*$ & $\mathrm{x}_{11}$ & $-2.11 \mathrm{E}-01$ & $1.43 \mathrm{E}+00$ & $-1.15 \mathrm{E}-02$ & $-1.03 \mathrm{E}+00$ & 4.67E-01 & $-9.63 \mathrm{E}-03$ & $-5.50 \mathrm{E}-03$ & $6.86 \mathrm{E}-02$ \\
\hline $\mathrm{x}_{4}$ & $*$ & $\mathrm{x}_{12}$ & $-6.22 \mathrm{E}-02$ & 7.93E-01 & $-1.15 \mathrm{E}-03$ & 4.86E-01 & $-6.24 \mathrm{E}-01$ & $-5.57 \mathrm{E}-03$ & $-3.94 \mathrm{E}-03$ & $5.44 \mathrm{E}-03$ \\
\hline$x_{4}$ & * & $\mathrm{x}_{13}$ & $-5.91 \mathrm{E}-02$ & $-2.45 \mathrm{E}+00$ & $3.14 \mathrm{E}-03$ & $-2.00 \mathrm{E}+00$ & $-2.12 \mathrm{E}+00$ & $-5.26 \mathrm{E}-03$ & $1.05 \mathrm{E}-03$ & $-3.69 \mathrm{E}-02$ \\
\hline $\mathrm{x}_{4}$ & $*$ & $\mathrm{x}_{14}$ & $-4.99 \mathrm{E}-03$ & $-1.49 \mathrm{E}+00$ & $-2.08 \mathrm{E}-03$ & $-6.55 \mathrm{E}-01$ & $-1.34 \mathrm{E}+00$ & $-4.02 \mathrm{E}-03$ & $5.99 \mathrm{E}-03$ & $-6.44 \mathrm{E}-03$ \\
\hline$x_{5}$ & * & $\mathrm{x}_{6}$ & $-1.21 \mathrm{E}-02$ & 7.92E-01 & $-7.28 \mathrm{E}-04$ & $2.60 \mathrm{E}-01$ & $-3.06 \mathrm{E}-01$ & $2.46 \mathrm{E}-04$ & $-2.65 \mathrm{E}-04$ & $1.41 \mathrm{E}-02$ \\
\hline $\mathrm{x}_{5}$ & $*$ & $\mathrm{x}_{7}$ & $-6.47 \mathrm{E}-03$ & $-1.08 \mathrm{E}-01$ & $1.59 \mathrm{E}-05$ & $-4.39 \mathrm{E}-02$ & $-2.13 \mathrm{E}-02$ & $-3.25 \mathrm{E}-05$ & $1.43 \mathrm{E}-04$ & $-1.21 \mathrm{E}-03$ \\
\hline $\mathrm{x}_{5}$ & $*$ & $\mathrm{x}_{8}$ & $-1.72 \mathrm{E}-03$ & $8.49 \mathrm{E}-02$ & $1.63 \mathrm{E}-04$ & 7.97E-02 & $2.93 \mathrm{E}-02$ & 4.29E-05 & $-2.48 \mathrm{E}-04$ & $1.20 \mathrm{E}-03$ \\
\hline $\mathrm{x}_{5}$ & $*$ & $\mathrm{X}_{9}$ & $5.63 \mathrm{E}-01$ & $3.81 \mathrm{E}+00$ & $-1.17 \mathrm{E}-02$ & $5.09 \mathrm{E}-01$ & $1.26 \mathrm{E}+00$ & $-5.48 \mathrm{E}-03$ & $-5.37 \mathrm{E}-03$ & $3.01 \mathrm{E}-02$ \\
\hline $\mathrm{x}_{5}$ & $*$ & $\mathrm{x}_{10}$ & $1.41 \mathrm{E}-01$ & $1.69 \mathrm{E}-01$ & $-1.50 \mathrm{E}-02$ & $-9.36 \mathrm{E}-01$ & $-1.56 \mathrm{E}+00$ & $-3.95 \mathrm{E}-03$ & $7.90 \mathrm{E}-04$ & $1.61 \mathrm{E}-02$ \\
\hline $\mathrm{x}_{5}$ & $*$ & $\mathrm{x}_{11}$ & $-9.79 \mathrm{E}-02$ & $-1.46 \mathrm{E}+00$ & $3.42 \mathrm{E}-03$ & $-5.85 \mathrm{E}-01$ & $-6.00 \mathrm{E}-01$ & $6.68 \mathrm{E}-04$ & $2.72 \mathrm{E}-03$ & $-1.93 \mathrm{E}-02$ \\
\hline $\mathrm{x}_{5}$ & $*$ & $\mathrm{x}_{12}$ & $4.26 \mathrm{E}-02$ & $4.26 \mathrm{E}-01$ & $2.43 \mathrm{E}-03$ & 3.03E-01 & $3.76 \mathrm{E}-01$ & $1.35 \mathrm{E}-03$ & $-5.66 \mathrm{E}-04$ & $3.04 \mathrm{E}-03$ \\
\hline $\mathrm{x}_{5}$ & $*$ & $\mathrm{x}_{13}$ & $1.26 \mathrm{E}-01$ & $1.67 \mathrm{E}+00$ & $-5.99 \mathrm{E}-03$ & $1.29 \mathrm{E}+00$ & $2.55 \mathrm{E}+00$ & $2.62 \mathrm{E}-03$ & $-3.62 \mathrm{E}-03$ & $5.26 \mathrm{E}-03$ \\
\hline $\mathrm{x}_{5}$ & $*$ & $\mathrm{x}_{14}$ & $-4.46 \mathrm{E}-02$ & $-2.36 \mathrm{E}-01$ & $-3.35 \mathrm{E}-03$ & $-2.02 \mathrm{E}-01$ & $-2.41 \mathrm{E}-01$ & $-1.43 \mathrm{E}-03$ & $1.11 \mathrm{E}-04$ & $2.14 \mathrm{E}-03$ \\
\hline $\mathrm{x}_{6}$ & $*$ & $\mathrm{x}_{7}$ & $-5.00 \mathrm{E}-02$ & $-1.65 \mathrm{E}+00$ & $6.61 \mathrm{E}-03$ & $-4.34 \mathrm{E}-01$ & $1.69 \mathrm{E}-02$ & $-1.29 \mathrm{E}-03$ & $3.07 \mathrm{E}-04$ & $-3.28 \mathrm{E}-02$ \\
\hline $\mathrm{x}_{6}$ & $*$ & $\mathrm{x}_{8}$ & $2.50 \mathrm{E}-01$ & $1.86 \mathrm{E}+00$ & $-4.63 \mathrm{E}-03$ & $-8.89 \mathrm{E}-02$ & $8.10 \mathrm{E}-01$ & $9.38 \mathrm{E}-03$ & $-4.57 \mathrm{E}-03$ & $3.08 \mathrm{E}-02$ \\
\hline $\mathrm{x}_{6}$ & $*$ & $\mathrm{X}_{9}$ & $1.35 \mathrm{E}-01$ & $1.26 \mathrm{E}+00$ & $-1.55 \mathrm{E}-04$ & $6.25 \mathrm{E}-01$ & $6.77 \mathrm{E}-01$ & $2.30 \mathrm{E}-03$ & $-1.05 \mathrm{E}-03$ & $1.70 \mathrm{E}-02$ \\
\hline $\mathrm{x}_{6}$ & $*$ & $\mathrm{x}_{10}$ & $4.00 \mathrm{E}-03$ & $1.88 \mathrm{E}-01$ & $1.27 \mathrm{E}-03$ & $2.40 \mathrm{E}-01$ & $2.66 \mathrm{E}-01$ & $6.56 \mathrm{E}-04$ & $-3.52 \mathrm{E}-04$ & $-5.11 \mathrm{E}-05$ \\
\hline $\mathrm{x}_{6}$ & $*$ & $\mathrm{x}_{11}$ & 4.13E-03 & $1.59 \mathrm{E}-02$ & $1.88 \mathrm{E}-03$ & 3.13E-01 & $2.82 \mathrm{E}-01$ & $1.55 \mathrm{E}-03$ & $7.78 \mathrm{E}-05$ & $-4.26 \mathrm{E}-03$ \\
\hline $\mathrm{x}_{6}$ & $*$ & $\mathrm{x}_{12}$ & $-5.16 \mathrm{E}-01$ & $-3.42 \mathrm{E}+00$ & $2.96 \mathrm{E}-02$ & $1.16 \mathrm{E}+00$ & $8.82 \mathrm{E}-01$ & $6.78 \mathrm{E}-03$ & $4.20 \mathrm{E}-03$ & $-8.92 \mathrm{E}-02$ \\
\hline $\mathrm{x}_{6}$ & $*$ & $\mathrm{x}_{13}$ & $4.61 \mathrm{E}-02$ & $-2.78 \mathrm{E}-02$ & $1.59 \mathrm{E}-03$ & $-2.67 \mathrm{E}-01$ & $-5.11 \mathrm{E}-01$ & 4.33E-05 & $6.28 \mathrm{E}-04$ & $1.34 \mathrm{E}-03$ \\
\hline $\mathrm{x}_{6}$ & $*$ & $\mathrm{x}_{14}$ & 4.31E-01 & $8.58 \mathrm{E}-01$ & $-5.72 \mathrm{E}-03$ & $-2.00 \mathrm{E}+00$ & $-1.92 \mathrm{E}+00$ & $-9.33 \mathrm{E}-03$ & $-1.36 \mathrm{E}-05$ & $-4.61 \mathrm{E}-03$ \\
\hline$x_{7}$ & $*$ & $\mathrm{x}_{8}$ & $-3.77 \mathrm{E}-03$ & $-1.78 \mathrm{E}-01$ & $-1.47 \mathrm{E}-04$ & $-1.16 \mathrm{E}-01$ & $-7.74 \mathrm{E}-02$ & $-5.59 \mathrm{E}-04$ & $6.44 \mathrm{E}-04$ & $-2.48 \mathrm{E}-03$ \\
\hline$x_{7}$ & $*$ & $\mathrm{x}_{9}$ & $-9.60 \mathrm{E}-01$ & $-6.46 \mathrm{E}+00$ & $1.59 \mathrm{E}-02$ & $-1.14 \mathrm{E}+00$ & $-2.88 \mathrm{E}+00$ & $3.51 \mathrm{E}-03$ & $8.81 \mathrm{E}-03$ & $-6.83 \mathrm{E}-02$ \\
\hline$x_{7}$ & * & $\mathrm{x}_{10}$ & $-2.60 \mathrm{E}-01$ & $3.89 \mathrm{E}-01$ & $2.79 \mathrm{E}-02$ & $2.86 \mathrm{E}+00$ & $2.45 \mathrm{E}+00$ & $8.60 \mathrm{E}-03$ & $-2.49 \mathrm{E}-03$ & $-2.77 \mathrm{E}-02$ \\
\hline$x_{7}$ & $*$ & $\mathrm{x}_{11}$ & 2.22E-01 & $2.79 \mathrm{E}+00$ & $-5.61 \mathrm{E}-03$ & $1.26 \mathrm{E}+00$ & $1.40 \mathrm{E}+00$ & $-3.11 \mathrm{E}-04$ & $-5.91 \mathrm{E}-03$ & $2.86 \mathrm{E}-02$ \\
\hline $\mathrm{x}_{7}$ & * & $\mathrm{x}_{12}$ & $-3.65 \mathrm{E}-02$ & $-6.97 \mathrm{E}-01$ & $-5.75 \mathrm{E}-03$ & $-6.27 \mathrm{E}-01$ & $-6.00 \mathrm{E}-01$ & $-2.49 \mathrm{E}-03$ & $1.22 \mathrm{E}-03$ & $-4.13 \mathrm{E}-03$ \\
\hline$x_{7}$ & $*$ & $\mathrm{x}_{13}$ & $1.61 \mathrm{E}-02$ & $-5.16 \mathrm{E}-01$ & $-4.93 \mathrm{E}-03$ & $-1.32 \mathrm{E}+00$ & $-2.06 \mathrm{E}+00$ & $-1.79 \mathrm{E}-03$ & $3.27 \mathrm{E}-03$ & $4.52 \mathrm{E}-02$ \\
\hline$x_{7}$ & * & $\mathrm{x}_{14}$ & 4.51E-02 & $6.10 \mathrm{E}-01$ & $6.40 \mathrm{E}-03$ & $5.02 \mathrm{E}-01$ & $5.05 \mathrm{E}-01$ & $2.93 \mathrm{E}-03$ & $-1.10 \mathrm{E}-03$ & $-2.93 \mathrm{E}-03$ \\
\hline $\mathrm{x}_{8}$ & $*$ & $\mathrm{X}_{9}$ & $-2.55 \mathrm{E}-01$ & $-1.15 \mathrm{E}+00$ & $8.12 \mathrm{E}-03$ & $3.06 \mathrm{E}-01$ & $3.35 \mathrm{E}-01$ & $3.47 \mathrm{E}-03$ & $-2.49 \mathrm{E}-04$ & $-1.21 \mathrm{E}-02$ \\
\hline $\mathrm{x}_{8}$ & * & $\mathrm{x}_{10}$ & $2.95 \mathrm{E}-02$ & $1.45 \mathrm{E}-01$ & $-1.33 \mathrm{E}-03$ & 8.80E-02 & $2.99 \mathrm{E}-01$ & $6.55 \mathrm{E}-04$ & $-6.25 \mathrm{E}-04$ & $1.60 \mathrm{E}-03$ \\
\hline $\mathrm{x}_{8}$ & $*$ & $\mathrm{x}_{11}$ & $-8.76 \mathrm{E}-02$ & $2.65 \mathrm{E}-01$ & $6.37 \mathrm{E}-04$ & $-6.67 \mathrm{E}-02$ & $-9.67 \mathrm{E}-01$ & $-1.88 \mathrm{E}-03$ & $4.86 \mathrm{E}-03$ & $1.54 \mathrm{E}-02$ \\
\hline $\mathrm{x}_{8}$ & $*$ & $\mathrm{x}_{12}$ & $-8.46 \mathrm{E}-02$ & $-3.29 \mathrm{E}-01$ & $4.11 \mathrm{E}-03$ & $2.56 \mathrm{E}-01$ & $2.21 \mathrm{E}-01$ & $3.73 \mathrm{E}-03$ & $-1.54 \mathrm{E}-03$ & $-4.40 \mathrm{E}-03$ \\
\hline $\mathrm{x}_{8}$ & $*$ & $\mathrm{x}_{13}$ & $-6.29 \mathrm{E}-01$ & $-4.82 \mathrm{E}+00$ & $1.75 \mathrm{E}-02$ & $-6.14 \mathrm{E}-01$ & $-1.46 \mathrm{E}+00$ & $5.86 \mathrm{E}-03$ & $5.12 \mathrm{E}-03$ & $-6.58 \mathrm{E}-02$ \\
\hline $\mathrm{x}_{8}$ & 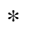 & $\mathrm{x}_{14}$ & $1.85 \mathrm{E}-01$ & $1.53 \mathrm{E}+00$ & $-3.30 \mathrm{E}-03$ & $4.54 \mathrm{E}-01$ & $-1.55 \mathrm{E}-01$ & $-3.43 \mathrm{E}-03$ & $-5.29 \mathrm{E}-04$ & $2.98 \mathrm{E}-02$ \\
\hline $\mathrm{x}_{9}$ & $*$ & $\mathrm{x}_{10}$ & $2.27 \mathrm{E}-03$ & $1.16 \mathrm{E}-05$ & $-1.10 \mathrm{E}-04$ & $-1.50 \mathrm{E}-02$ & $-4.00 \mathrm{E}-02$ & $3.55 \mathrm{E}-06$ & $8.75 \mathrm{E}-05$ & 4.13E-04 \\
\hline $\mathrm{X}_{9}$ & * & $\mathrm{x}_{11}$ & $-8.15 \mathrm{E}-04$ & $-1.20 \mathrm{E}-01$ & $1.38 \mathrm{E}-03$ & $-5.29 \mathrm{E}-02$ & $-2.51 \mathrm{E}-01$ & $9.05 \mathrm{E}-05$ & $5.28 \mathrm{E}-04$ & $-1.30 \mathrm{E}-03$ \\
\hline $\mathrm{X}_{9}$ & $*$ & $\mathrm{x}_{12}$ & $6.65 \mathrm{E}-01$ & $4.90 \mathrm{E}+00$ & $5.48 \mathrm{E}-03$ & $2.30 \mathrm{E}+00$ & $2.58 \mathrm{E}+00$ & $1.11 \mathrm{E}-02$ & $-5.03 \mathrm{E}-03$ & $7.53 \mathrm{E}-02$ \\
\hline
\end{tabular}


2021.

\begin{tabular}{|c|c|c|c|c|c|c|c|c|c|c|}
\hline $\mathrm{X}_{9}$ & $*$ & $\mathrm{x}_{13}$ & $2.43 \mathrm{E}-02$ & $2.39 \mathrm{E}-01$ & $6.22 \mathrm{E}-05$ & $1.38 \mathrm{E}-01$ & $1.59 \mathrm{E}-01$ & 4.43E-04 & $-2.82 \mathrm{E}-04$ & $2.83 \mathrm{E}-03$ \\
\hline $\mathrm{X}_{9}$ & $*$ & $\mathrm{x}_{14}$ & $6.89 \mathrm{E}-01$ & $4.98 \mathrm{E}+00$ & $3.58 \mathrm{E}-03$ & $1.95 \mathrm{E}+00$ & $2.06 \mathrm{E}+00$ & 8.23E-03 & $-4.67 \mathrm{E}-03$ & 7.37E-02 \\
\hline $\mathrm{x}_{10}$ & $*$ & $\mathrm{x}_{11}$ & $-3.60 \mathrm{E}-03$ & $-2.07 \mathrm{E}-01$ & $-1.15 \mathrm{E}-03$ & $-2.31 \mathrm{E}-01$ & $-3.74 \mathrm{E}-01$ & $-4.30 \mathrm{E}-04$ & $6.76 \mathrm{E}-04$ & $3.63 \mathrm{E}-05$ \\
\hline $\mathrm{x}_{10}$ & $*$ & $\mathrm{x}_{12}$ & $6.19 \mathrm{E}-02$ & 3.69E-01 & $1.07 \mathrm{E}-03$ & $1.63 \mathrm{E}-01$ & $2.79 \mathrm{E}-01$ & 4.69E-04 & $-8.75 \mathrm{E}-04$ & $1.76 \mathrm{E}-03$ \\
\hline $\mathrm{x}_{10}$ & $*$ & $\mathrm{x}_{13}$ & $-2.36 \mathrm{E}-03$ & $-3.10 \mathrm{E}-02$ & $1.21 \mathrm{E}-04$ & $-2.36 \mathrm{E}-02$ & $-8.86 \mathrm{E}-03$ & $-1.15 \mathrm{E}-04$ & $-4.72 \mathrm{E}-05$ & $-3.10 \mathrm{E}-04$ \\
\hline $\mathrm{X}_{10}$ & $*$ & $\mathrm{x}_{14}$ & 3.98E-02 & $1.07 \mathrm{E}-01$ & 7.89E-04 & $-1.71 \mathrm{E}-02$ & $5.30 \mathrm{E}-02$ & $-1.64 \mathrm{E}-04$ & $-4.40 \mathrm{E}-04$ & $-1.20 \mathrm{E}-03$ \\
\hline $\mathrm{x}_{11}$ & $*$ & $\mathrm{x}_{12}$ & $-1.72 \mathrm{E}-01$ & 2.33E-01 & $-5.33 \mathrm{E}-03$ & $1.44 \mathrm{E}-01$ & $-4.63 \mathrm{E}-01$ & $6.52 \mathrm{E}-05$ & 4.24E-03 & $1.54 \mathrm{E}-02$ \\
\hline $\mathrm{x}_{11}$ & $*$ & $\mathrm{x}_{13}$ & $8.75 \mathrm{E}-02$ & $6.48 \mathrm{E}-01$ & $-3.75 \mathrm{E}-04$ & $2.86 \mathrm{E}-01$ & $3.24 \mathrm{E}-01$ & $9.45 \mathrm{E}-04$ & $-5.15 \mathrm{E}-04$ & 7.72E-03 \\
\hline $\mathrm{x}_{11}$ & $*$ & $\mathrm{x}_{14}$ & $1.81 \mathrm{E}-01$ & $2.33 \mathrm{E}-01$ & $-1.78 \mathrm{E}-02$ & $-1.05 \mathrm{E}+00$ & $3.93 \mathrm{E}-02$ & $-1.59 \mathrm{E}-03$ & $1.46 \mathrm{E}-03$ & $2.61 \mathrm{E}-02$ \\
\hline $\mathrm{X}_{12}$ & $*$ & $\mathrm{x}_{13}$ & $-4.80 \mathrm{E}-01$ & $-6.24 \mathrm{E}+00$ & $2.04 \mathrm{E}-02$ & $-3.44 \mathrm{E}+00$ & $-4.58 \mathrm{E}+00$ & $-5.57 \mathrm{E}-03$ & $5.13 \mathrm{E}-03$ & $-8.35 \mathrm{E}-02$ \\
\hline $\mathrm{x}_{12}$ & $*$ & $\mathrm{x}_{14}$ & $-9.00 \mathrm{E}-01$ & $-4.25 \mathrm{E}+00$ & 1.07E-02 & $-4.48 \mathrm{E}-01$ & $-4.43 \mathrm{E}-01$ & $-8.04 \mathrm{E}-03$ & $3.22 \mathrm{E}-03$ & $-6.27 \mathrm{E}-02$ \\
\hline \multirow[t]{3}{*}{$\mathrm{x}_{13}$} & $*$ & $\mathrm{x}_{14}$ & $-3.11 \mathrm{E}-01$ & $-4.36 \mathrm{E}+00$ & $1.43 \mathrm{E}-02$ & $-2.46 \mathrm{E}+00$ & $-3.04 \mathrm{E}+00$ & $-3.42 \mathrm{E}-03$ & $3.25 \mathrm{E}-03$ & $-6.13 \mathrm{E}-02$ \\
\hline & $\mathrm{c}$ & & $1.59 \mathrm{E}-02$ & $1.49 \mathrm{E}-01$ & $1.11 \mathrm{E}-04$ & $6.45 \mathrm{E}-02$ & 7.19E-02 & $1.49 \mathrm{E}-04$ & $-8.98 \mathrm{E}-05$ & $2.06 \mathrm{E}-03$ \\
\hline & $r^{2}$ & & 1 & 1 & 1 & 1 & 1 & 1 & 1 & 1 \\
\hline
\end{tabular}

Table 5. The $\mathrm{x}$ and $\mathrm{y}$ variables, their coefficient and determination coefficient of equations belonging non-linear models yielded from the data collected Station 2

\begin{tabular}{|c|c|c|c|c|c|c|c|c|c|c|}
\hline & & & $\mathrm{y}_{1}$ & $\mathrm{y}_{2}$ & $\mathrm{y}_{3}$ & $\mathrm{y}_{4}$ & $\mathrm{y}_{5}$ & $\mathrm{y}_{6}$ & $\mathrm{y}_{7}$ & $\mathrm{y}_{8}$ \\
\hline & $\mathrm{x}_{1}$ & & $1.15 \mathrm{E}-04$ & $-8.45 \mathrm{E}-02$ & $5.08 \mathrm{E}-05$ & $1.09 \mathrm{E}-03$ & $-2.98 \mathrm{E}-04$ & $1.27 \mathrm{E}-06$ & $-9.24 \mathrm{E}-08$ & $1.21 \mathrm{E}-05$ \\
\hline & $\mathrm{x}_{2}$ & & $1.08 \mathrm{E}-05$ & $-4.97 \mathrm{E}-03$ & $5.80 \mathrm{E}-06$ & $1.47 \mathrm{E}-04$ & 4.07E-05 & $1.70 \mathrm{E}-07$ & 7.85E-09 & $3.52 \mathrm{E}-06$ \\
\hline & $\mathrm{x}_{3}$ & & $1.69 \mathrm{E}-04$ & $9.67 \mathrm{E}-02$ & $6.95 \mathrm{E}-05$ & $1.61 \mathrm{E}-03$ & $1.74 \mathrm{E}-03$ & 2.83E-06 & $4.58 \mathrm{E}-07$ & $1.30 \mathrm{E}-04$ \\
\hline & $\mathrm{x}_{4}$ & & $2.17 \mathrm{E}-04$ & $1.64 \mathrm{E}-01$ & $6.21 \mathrm{E}-05$ & $1.99 \mathrm{E}-03$ & $1.13 \mathrm{E}-03$ & $2.59 \mathrm{E}-06$ & $3.29 \mathrm{E}-07$ & $1.18 \mathrm{E}-04$ \\
\hline & $\mathrm{x}_{5}$ & & 7.12E-04 & $2.31 \mathrm{E}+00$ & $1.60 \mathrm{E}-03$ & $5.63 \mathrm{E}-02$ & $-2.69 \mathrm{E}-02$ & 4.78E-05 & $-8.19 \mathrm{E}-05$ & $3.16 \mathrm{E}-03$ \\
\hline & $\mathrm{x}_{6}$ & & $1.94 \mathrm{E}-04$ & $-1.75 \mathrm{E}-02$ & $6.70 \mathrm{E}-05$ & $1.89 \mathrm{E}-03$ & $1.08 \mathrm{E}-03$ & $3.18 \mathrm{E}-06$ & $2.44 \mathrm{E}-07$ & $1.28 \mathrm{E}-04$ \\
\hline & $\mathrm{x}_{7}$ & & $2.20 \mathrm{E}-03$ & $-5.55 \mathrm{E}-01$ & $8.50 \mathrm{E}-04$ & $2.21 \mathrm{E}-02$ & $1.41 \mathrm{E}-02$ & 4.01E-05 & $2.20 \mathrm{E}-06$ & $1.83 \mathrm{E}-03$ \\
\hline & $\mathrm{x}_{8}$ & & $2.15 \mathrm{E}-04$ & $-6.74 \mathrm{E}-03$ & $1.31 \mathrm{E}-04$ & $3.87 \mathrm{E}-03$ & $1.20 \mathrm{E}-03$ & $2.31 \mathrm{E}-07$ & $-3.15 \mathrm{E}-07$ & $2.24 \mathrm{E}-04$ \\
\hline & $\mathrm{X}_{9}$ & & $9.02 \mathrm{E}-06$ & $-1.00 \mathrm{E}-02$ & $4.26 \mathrm{E}-06$ & $1.04 \mathrm{E}-04$ & $4.56 \mathrm{E}-05$ & $1.87 \mathrm{E}-07$ & $1.66 \mathrm{E}-08$ & $3.91 \mathrm{E}-06$ \\
\hline & $\mathrm{x}_{10}$ & & $-1.07 \mathrm{E}-06$ & $-2.03 \mathrm{E}-04$ & $3.76 \mathrm{E}-08$ & $-4.40 \mathrm{E}-06$ & $-1.48 \mathrm{E}-07$ & $-2.80 \mathrm{E}-08$ & $-4.71 E-09$ & $-3.59 \mathrm{E}-07$ \\
\hline & $\mathrm{x}_{11}$ & & $1.03 \mathrm{E}-04$ & $2.09 \mathrm{E}-01$ & $2.25 \mathrm{E}-05$ & $1.12 \mathrm{E}-03$ & $6.04 \mathrm{E}-04$ & $5.57 \mathrm{E}-07$ & $1.32 \mathrm{E}-07$ & $6.16 \mathrm{E}-05$ \\
\hline & $\mathrm{x}_{12}$ & & $2.87 \mathrm{E}-04$ & $1.55 \mathrm{E}-01$ & $8.56 \mathrm{E}-05$ & $2.64 \mathrm{E}-03$ & $1.20 \mathrm{E}-03$ & $2.70 \mathrm{E}-06$ & $6.63 \mathrm{E}-08$ & $1.56 \mathrm{E}-04$ \\
\hline & $\mathrm{x}_{13}$ & & $1.99 \mathrm{E}-05$ & $-1.10 \mathrm{E}-02$ & 7.23E-06 & $1.90 \mathrm{E}-04$ & $1.07 \mathrm{E}-04$ & $3.57 \mathrm{E}-07$ & 2.38E-08 & $1.68 \mathrm{E}-05$ \\
\hline & $\mathrm{x}_{14}$ & & $2.33 \mathrm{E}-04$ & $1.44 \mathrm{E}-01$ & $7.95 \mathrm{E}-05$ & $2.18 \mathrm{E}-03$ & $1.06 \mathrm{E}-03$ & $2.65 \mathrm{E}-06$ & 2.37E-07 & $1.58 \mathrm{E}-04$ \\
\hline $\mathrm{x}_{1}$ & $*$ & $\mathrm{x}_{1}$ & $2.02 \mathrm{E}-03$ & $-2.50 \mathrm{E}-02$ & $4.86 \mathrm{E}-04$ & $-4.50 \mathrm{E}-03$ & $-7.67 \mathrm{E}-03$ & $5.89 \mathrm{E}-06$ & $-1.61 \mathrm{E}-05$ & $2.84 \mathrm{E}-03$ \\
\hline $\mathrm{x}_{2}$ & $*$ & $\mathrm{x}_{2}$ & $1.20 \mathrm{E}-05$ & $-9.78 \mathrm{E}-04$ & 6.33E-06 & $1.54 \mathrm{E}-04$ & $1.35 \mathrm{E}-04$ & $2.20 \mathrm{E}-07$ & $3.55 \mathrm{E}-08$ & $1.19 \mathrm{E}-05$ \\
\hline $\mathrm{x}_{3}$ & $*$ & $\mathrm{x}_{3}$ & $-3.03 E-03$ & $1.42 \mathrm{E}+00$ & $-2.36 \mathrm{E}-04$ & $-3.08 \mathrm{E}-02$ & $4.31 \mathrm{E}-03$ & $-3.39 \mathrm{E}-05$ & $-1.32 \mathrm{E}-06$ & $-1.98 \mathrm{E}-03$ \\
\hline $\mathrm{X}_{4}$ & $*$ & $\mathrm{X}_{4}$ & $5.07 \mathrm{E}-04$ & $-5.59 \mathrm{E}-02$ & $5.75 \mathrm{E}-04$ & $5.43 \mathrm{E}-03$ & $1.82 \mathrm{E}-02$ & $3.15 \mathrm{E}-05$ & 7.08E-06 & $1.20 \mathrm{E}-03$ \\
\hline $\mathrm{X}_{5}$ & $*$ & $\mathrm{x}_{5}$ & $5.84 \mathrm{E}-08$ & $-1.28 \mathrm{E}-04$ & $-1.11 \mathrm{E}-09$ & $-4.85 \mathrm{E}-08$ & $1.77 \mathrm{E}-07$ & $-4.96 \mathrm{E}-11$ & $-1.10 \mathrm{E}-11$ & 4.87E-09 \\
\hline $\mathrm{x}_{6}$ & $*$ & $\mathrm{x}_{6}$ & $1.54 \mathrm{E}-03$ & $-2.10 \mathrm{E}-01$ & 5.37E-04 & $1.48 \mathrm{E}-02$ & $9.27 \mathrm{E}-03$ & 2.64E-05 & 2.94E-06 & $9.55 \mathrm{E}-04$ \\
\hline$x_{7}$ & $*$ & $\mathrm{x}_{7}$ & $8.05 \mathrm{E}-05$ & $2.71 \mathrm{E}-02$ & $-9.27 \mathrm{E}-05$ & $-8.16 \mathrm{E}-04$ & $-3.80 \mathrm{E}-04$ & $-3.83 \mathrm{E}-06$ & $-6.33 \mathrm{E}-07$ & $-3.23 \mathrm{E}-05$ \\
\hline $\mathrm{X}_{8}$ & $*$ & $\mathrm{x}_{8}$ & $-4.91 \mathrm{E}-04$ & $4.81 \mathrm{E}-01$ & $-4.06 \mathrm{E}-05$ & $-1.55 \mathrm{E}-03$ & $5.17 \mathrm{E}-03$ & $1.18 \mathrm{E}-06$ & $2.02 \mathrm{E}-06$ & $1.80 \mathrm{E}-04$ \\
\hline $\mathrm{X}_{9}$ & $*$ & $\mathrm{X}_{9}$ & $9.40 \mathrm{E}-07$ & $-1.12 \mathrm{E}-02$ & $1.89 \mathrm{E}-06$ & $1.67 \mathrm{E}-05$ & 4.35E-05 & $1.16 \mathrm{E}-07$ & 4.15E-08 & $-2.95 \mathrm{E}-06$ \\
\hline $\mathrm{x}_{10}$ & $*$ & $\mathrm{x}_{10}$ & $1.67 \mathrm{E}-16$ & $1.17 \mathrm{E}-13$ & $1.98 \mathrm{E}-17$ & $1.46 \mathrm{E}-15$ & $-6.71 \mathrm{E}-17$ & $9.66 \mathrm{E}-19$ & $-3.94 \mathrm{E}-19$ & $8.75 \mathrm{E}-17$ \\
\hline $\mathrm{x}_{11}$ & $*$ & $\mathrm{x}_{11}$ & $5.78 \mathrm{E}-03$ & $2.99 \mathrm{E}+00$ & $-2.43 \mathrm{E}-04$ & $4.99 \mathrm{E}-02$ & $-4.62 \mathrm{E}-02$ & $-1.31 \mathrm{E}-06$ & $-3.11 \mathrm{E}-05$ & $1.19 \mathrm{E}-03$ \\
\hline $\mathrm{x}_{12}$ & $*$ & $\mathrm{x}_{12}$ & $2.54 \mathrm{E}-03$ & $5.41 \mathrm{E}+00$ & $2.50 \mathrm{E}-04$ & $1.01 \mathrm{E}-03$ & $3.24 \mathrm{E}-03$ & $-1.74 \mathrm{E}-05$ & $-4.69 \mathrm{E}-06$ & $3.03 \mathrm{E}-04$ \\
\hline $\mathrm{x}_{13}$ & $*$ & $\mathrm{x}_{13}$ & $1.80 \mathrm{E}-05$ & $-1.45 \mathrm{E}-02$ & 6.33E-06 & $1.52 \mathrm{E}-04$ & $1.27 \mathrm{E}-04$ & $3.95 \mathrm{E}-07$ & 4.84E-08 & $1.71 \mathrm{E}-05$ \\
\hline
\end{tabular}


quality

\begin{tabular}{|c|c|c|c|c|c|c|c|c|c|c|}
\hline $\mathrm{x}_{14}$ & & $\mathrm{x}_{14}$ & $.79 \mathrm{E}-04$ & $4.22 \mathrm{E}+00$ & $-7.04 \mathrm{E}-05$ & -02 & $.19 \mathrm{E}-02$ & 05 & $-1.11 \mathrm{E}-05$ & -84 \\
\hline $\mathrm{x}_{1}$ & * & $\mathrm{x}_{2}$ & $1.96 \mathrm{E}-04$ & $-9.47 \mathrm{E}-03$ & 7.78E-05 & $1.94 \mathrm{E}-03$ & $1.82 \mathrm{E}-03$ & $3.60 \mathrm{E}-06$ & $3.21 \mathrm{E}-07$ & $1.60 \mathrm{E}-04$ \\
\hline $\mathrm{x}_{1}$ & & $\alpha_{j}$ & $.20 \mathrm{E}-04$ & $-6.92 \mathrm{E}-01$ & $-5.71 \mathrm{E}-05$ & $2.04 \mathrm{E}-02$ & $-9.76 \mathrm{E}-03$ & $1.16 \mathrm{E}-05$ & $-1.34 \mathrm{E}-05$ & $2.00 \mathrm{E}-04$ \\
\hline $\mathrm{x}_{1}$ & & $\mathrm{x}_{4}$ & 7.68E-03 & $9.93 \mathrm{E}+00$ & $1.13 \mathrm{E}-03$ & 5.19E-02 & $3.71 \mathrm{E}-02$ & $6.59 \mathrm{E}-05$ & $1.20 \mathrm{E}-05$ & $4.65 \mathrm{E}-03$ \\
\hline $\mathrm{x}_{1}$ & & $\mathrm{x}_{5}$ & $65 \mathrm{E}-05$ & $.07 \mathrm{E}-01$ & $.69 \mathrm{E}-05$ & $.99 \mathrm{E}-04$ & $1.21 \mathrm{E}-04$ & $2.22 \mathrm{E}-07$ & $5.94 \mathrm{E}-08$ & $-3.84 \mathrm{E}-05$ \\
\hline $\mathrm{x}_{1}$ & & $\mathrm{x}_{6}$ & $2.28 \mathrm{E}-03$ & $-5.09 \mathrm{E}-02$ & 7.10E-04 & $1.80 \mathrm{E}-02$ & $1.35 \mathrm{E}-02$ & $3.53 \mathrm{E}-05$ & 3.91E-06 & $1.66 \mathrm{E}-03$ \\
\hline $\mathrm{x}_{1}$ & & $\mathrm{x}_{7}$ & 30E-03 & $53 \mathrm{E}+00$ & 5.8 & E-03 & $-7.88 \mathrm{E}-03$ & $.34 \mathrm{E}-05$ & $21 \mathrm{E}-06$ & $6.74 \mathrm{E}-$ \\
\hline $\mathrm{x}_{1}$ & 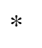 & $\mathrm{x}_{8}$ & $1.39 \mathrm{E}-02$ & $-1.60 \mathrm{E}+01$ & 2.67E-04 & 8.03E-02 & $-7.44 \mathrm{E}-02$ & $1.89 \mathrm{E}-04$ & $-4.53 \mathrm{E}-05$ & $6.26 \mathrm{E}-03$ \\
\hline $\mathrm{x}_{1}$ & * & $\mathrm{X}_{9}$ & 44E-04 & E-01 & $5.74 \mathrm{E}$ & E-03 & $26 \mathrm{E}-03$ & $3.11 \mathrm{E}-06$ & $7 \mathrm{E}-07$ & $1.08 \mathrm{E}-04$ \\
\hline $\mathrm{x}_{1}$ & , & $\mathrm{x}_{10}$ & $-3.85 \mathrm{E}-05$ & $-3.00 \mathrm{E}-02$ & $-5.48 \mathrm{E}-06$ & $-2.63 \mathrm{E}-04$ & $-1.94 \mathrm{E}-04$ & $-6.81 \mathrm{E}-07$ & $-1.90 \mathrm{E}-07$ & $-1.56 \mathrm{E}-05$ \\
\hline $\mathrm{x}_{1}$ & * & $\mathrm{x}_{11}$ & $.35 \mathrm{E}-03$ & $9.86 \mathrm{E}+00$ & $3.12 \mathrm{E}-04$ & $9.00 \mathrm{E}-03$ & $3.14 \mathrm{E}-02$ & $-5.28 \mathrm{E}-05$ & $2.02 \mathrm{E}-05$ & $-1.34 \mathrm{E}-03$ \\
\hline $\mathrm{x}_{1}$ & 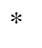 & $\mathrm{x}_{12}$ & 2.63E-03 & $4.07 \mathrm{E}+00$ & 4.28E-04 & $5.11 \mathrm{E}-03$ & $-1.17 \mathrm{E}-02$ & $-2.01 \mathrm{E}-05$ & $-1.84 \mathrm{E}-05$ & $1.11 \mathrm{E}-03$ \\
\hline $\mathrm{x}_{1}$ & & $\mathrm{x}_{13}$ & $1.61 \mathrm{E}-04$ & $-4.88 \mathrm{E}-01$ & $5.81 \mathrm{E}-05$ & $1.18 \mathrm{E}-03$ & 7.14E-04 & $5.15 \mathrm{E}-06$ & $2.51 \mathrm{E}-07$ & $2.14 \mathrm{E}-04$ \\
\hline $\mathrm{x}_{1}$ & & $\mathrm{x}_{14}$ & $6.23 \mathrm{E}-04$ & $6.31 \mathrm{E}-01$ & $-2.11 \mathrm{E}-05$ & $-9.58 \mathrm{E}-03$ & $-2.80 \mathrm{E}-02$ & $-1.69 \mathrm{E}-05$ & $-2.05 \mathrm{E}-05$ & $6.56 \mathrm{E}-04$ \\
\hline $\mathrm{x}_{2}$ & * & $\mathrm{x}_{3}$ & $1.03 \mathrm{E}-04$ & 1 & 7.1 & 3 & $2.36 \mathrm{E}-\mathrm{C}$ & $2.12 \mathrm{E}-06$ & 07 & 1 \\
\hline $\mathrm{x}_{2}$ & & $\mathrm{x}_{4}$ & $1.37 \mathrm{E}-04$ & $-7.62 \mathrm{E}-02$ & $5.01 \mathrm{E}-05$ & $1.50 \mathrm{E}-03$ & 7.32E-04 & $2.50 \mathrm{E}-06$ & $5.01 \mathrm{E}-08$ & $1.11 \mathrm{E}-04$ \\
\hline $\mathrm{x}_{2}$ & * & $\mathrm{x}_{5}$ & $8.41 \mathrm{E}-04$ & $-4.02 \mathrm{E}-01$ & $-1.90 \mathrm{E}-04$ & 2.35E-03 & $-1.80 \mathrm{E}-03$ & $-1.60 \mathrm{E}-06$ & $-3.48 \mathrm{E}-06$ & $1.23 \mathrm{E}-03$ \\
\hline$x_{2}$ & & $\mathrm{x}_{6}$ & $1.53 \mathrm{E}-04$ & $-3.01 \mathrm{E}-02$ & $5.76 \mathrm{E}-05$ & $1.63 \mathrm{E}-03$ & $9.79 \mathrm{E}-04$ & $2.65 \mathrm{E}-06$ & $1.72 \mathrm{E}-07$ & $1.09 \mathrm{E}-04$ \\
\hline $\mathrm{x}_{2}$ & & $\mathrm{x}_{7}$ & $2.48 \mathrm{E}-03$ & 9.9 & 9.7 & 2 & 1.9 & 55 & -06 & 2.4 \\
\hline $\mathrm{x}_{2}$ & $*$ & $\mathrm{x}_{8}$ & 4 & -01 & 1.2 & $1.74 \mathrm{E}-03$ & $3.83 \mathrm{E}-03$ & $-6.75 \mathrm{E}-06$ & $1.46 \mathrm{E}-06$ & $-1.92 \mathrm{E}-06$ \\
\hline $\mathrm{x}_{2}$ & $*$ & $\mathrm{X}_{9}$ & $5.23 \mathrm{E}-06$ & $-1.28 \mathrm{E}-02$ & 3.39E-06 & 8.09E-05 & $2.18 \mathrm{E}-05$ & $1.37 \mathrm{E}-07$ & $-1.86 \mathrm{E}-09$ & $2.14 \mathrm{E}-06$ \\
\hline $\mathrm{x}_{2}$ & * & $\mathrm{x}_{10}$ & .05 & $5.06 \mathrm{E}-04$ & -1.0 & $\mathrm{E}-07$ & $\mathrm{E}-05$ & $-3.75 \mathrm{E}-08$ & $-1.14 \mathrm{E}-08$ & $-1.08 \mathrm{E}-06$ \\
\hline $\mathrm{x}_{2}$ & * & $\mathrm{x}_{11}$ & $9.91 \mathrm{E}-05$ & $1.51 \mathrm{E}-02$ & $1.82 \mathrm{E}-05$ & $1.01 \mathrm{E}-03$ & $8.75 \mathrm{E}-05$ & $1.34 \mathrm{E}-06$ & $-3.20 \mathrm{E}-07$ & $1.00 \mathrm{E}-04$ \\
\hline $\mathrm{x}_{2}$ & * & $\mathrm{x}_{12}$ & + & 2 & 7.8 & 3 & 1.6 & 2.6 & E-07 & 1.2 \\
\hline $\mathrm{x}_{2}$ & * & $\mathrm{x}_{13}$ & $2.00 \mathrm{E}-05$ & $-8.03 \mathrm{E}-03$ & $6.66 \mathrm{E}-06$ & $2.04 \mathrm{E}-04$ & $8.48 \mathrm{E}-05$ & 3.19E-07 & $8.90 \mathrm{E}-09$ & $1.40 \mathrm{E}-05$ \\
\hline $\mathrm{x}_{2}$ & $*$ & $\mathrm{x}_{14}$ & $1 .$. & 1 & $8.16 \mathrm{E}-05$ & 3 & 2. & 2.2 & 07 & 1.3 \\
\hline $\mathrm{x}_{3}$ & $*$ & $\mathrm{x}_{4}$ & $5.64 \mathrm{E}-03$ & $9.13 \mathrm{E}+00$ & 7.07E-04 & $3.59 \mathrm{E}-02$ & $3.68 \mathrm{E}-02$ & $4.89 \mathrm{E}-05$ & $1.59 \mathrm{E}-05$ & $3.12 \mathrm{E}-03$ \\
\hline $\mathrm{x}_{3}$ & & $\mathrm{x}_{5}$ & ; & 1 & $1.54 \mathrm{E}-05$ & 4 & -03 & $-7.08 \mathrm{E}-09$ & 97 & $9.36 \mathrm{E}-0$ \\
\hline $\mathrm{x}_{3}$ & * & $\mathrm{x}_{6}$ & $29 \mathrm{E}-03$ & 7.09E-01 & $5.49 \mathrm{E}-04$ & $.18 \mathrm{E}-02$ & $1.57 \mathrm{E}-02$ & 2.43E-05 & $6.28 \mathrm{E}-06$ & $8.61 \mathrm{E}-04$ \\
\hline $\mathrm{x}_{3}$ & * & $\mathrm{x}_{7}$ & $.57 \mathrm{E}$ & 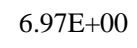 & $-5.22 \mathrm{E}-04$ & 2 & 6.7 & $1.12 \mathrm{E}-05$ & & -5.9 \\
\hline $\mathrm{x}_{3}$ & $*$ & $\mathrm{x}_{8}$ & 8.13E-04 & $-5.19 \mathrm{E}+00$ & $4.16 \mathrm{E}-04$ & $1.19 \mathrm{E}-02$ & $1.29 \mathrm{E}-02$ & $-4.76 \mathrm{E}-05$ & 5.01E-07 & $4.16 \mathrm{E}-03$ \\
\hline $\mathrm{x}_{3}$ & * & $\mathrm{X}_{9}$ & $8.86 \mathrm{E}-05$ & $-1.03 \mathrm{E}-01$ & 4.71E-05 & 4 & 3 & $2.25 \mathrm{E}-06$ & 77 & 5.6 \\
\hline $\mathrm{x}_{3}$ & 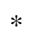 & $\mathrm{x}_{10}$ & $.05 \mathrm{E}-05$ & $-1.64 \mathrm{E}-02$ & $-5.25 \mathrm{E}-06$ & $-2.67 \mathrm{E}-04$ & $-1.44 \mathrm{E}-04$ & $-7.46 \mathrm{E}-07$ & $-1.62 \mathrm{E}-07$ & $-1.89 \mathrm{E}-05$ \\
\hline $\mathrm{x}_{3}$ & * & $\mathrm{x}_{11}$ & $.78 \mathrm{E}$ & 9.4 & 3. & 2 & 2 & 5 & 05 & -6 . \\
\hline $\mathrm{x}_{3}$ & * & $\mathrm{x}_{12}$ & $2.47 \mathrm{E}-04$ & $5.74 \mathrm{E}+00$ & $1.16 \mathrm{E}-04$ & $-4.73 \mathrm{E}-03$ & $-5.40 \mathrm{E}-03$ & $-5.05 \mathrm{E}-05$ & $-1.12 \mathrm{E}-05$ & $-1.17 \mathrm{E}-03$ \\
\hline $\mathrm{x}_{3}$ & * & $\mathrm{x}_{13}$ & $4 \angle \mathrm{E}$ & -3.8 & $4.96 \mathrm{E}-05$ & 4 & $1.19 \mathrm{E}-03$ & 4. & 97 & 1.7 \\
\hline $\mathrm{x}_{3}$ & 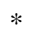 & $\mathrm{x}_{14}$ & $-2.02 \mathrm{E}-03$ & $3.14 \mathrm{E}+00$ & $-2.07 \mathrm{E}-04$ & $-2.23 \mathrm{E}-02$ & $-1.00 \mathrm{E}-02$ & $-4.83 \mathrm{E}-05$ & $-6.34 \mathrm{E}-06$ & $-1.56 \mathrm{E}-03$ \\
\hline $\mathrm{x}_{4}$ & * & $\mathrm{x}$ & $7 \mathrm{E}-0$ & $1.25 \mathrm{E}-01$ & -1.04 & -05 & 1.7 & 2.7 & -2 & $1.49 \mathrm{E}$ \\
\hline $\mathrm{x}_{4}$ & * & $\mathrm{x}_{6}$ & $1.70 \mathrm{E}-03$ & $1.05 \mathrm{E}+00$ & 5.12 & 1.5 & 1.05 & $2.36 \mathrm{E}-05$ & 4.33E-06 & $8.30 \mathrm{E}-04$ \\
\hline $\mathrm{x}_{4}$ & * & $\mathrm{x}_{7}$ & $63 \mathrm{E}-\mathrm{C}$ & $.33 \mathrm{E}+00$ & $-2.89 \mathrm{~F}$ & E-02 & -2.35 & -1 & $1.43 \mathrm{E}-06$ & $-3.09 \mathrm{E}-03$ \\
\hline $\mathrm{x}_{4}$ & $*$ & $\mathrm{x}_{8}$ & 8.13E-04 & $-5.19 \mathrm{E}+00$ & 4.16 & $1.19 \mathrm{E}-02$ & 1.2 & $-4.76 \mathrm{E}-05$ & 5.01E-07 & $4.16 \mathrm{E}-03$ \\
\hline $\mathrm{x}_{4}$ & $*$ & $\mathrm{X}_{9}$ & $3.68 \mathrm{E}-0$ & $-1.06 \mathrm{E}-01$ & $1.75 \mathrm{E}-0$ & $-8.64 \mathrm{E}-05$ & 4.37E-04 & $8.95 \mathrm{E}-07$ & $5.18 \mathrm{E}-07$ & 05 \\
\hline $\mathrm{x}_{4}$ & $*$ & $\mathrm{x}_{10}$ & 95 & $-1.75 \mathrm{E}-02$ & -2.9 & -1.8 & -1.3 & $-2.91 \mathrm{E}-07$ & $-4.03 \mathrm{E}-08$ & $-4.55 \mathrm{E}-06$ \\
\hline $\mathrm{x}_{4}$ & $*$ & $\mathrm{x}_{11}$ & $.002-03$ & To & + & 03 & 49E-02 & 05 & $-6.77 \mathrm{E}-06$ & $-2.20 \mathrm{E}-03$ \\
\hline$x_{4}$ & & $\mathrm{x}_{12}$ & -03 & $9.33 \mathrm{E}+00$ & -03 & $\mathrm{E}-02$ & -02 & 5.45E-05 & $5.82 \mathrm{E}-06$ & $5.58 \mathrm{E}-03$ \\
\hline
\end{tabular}




\begin{tabular}{|c|c|c|c|c|c|c|c|c|c|c|}
\hline $\mathrm{x}_{4}$ & 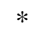 & $\mathrm{x}_{13}$ & $1.35 \mathrm{E}-04$ & $1.34 \mathrm{E}-02$ & $5.27 \mathrm{E}-05$ & $1.17 \mathrm{E}-03$ & $9.24 \mathrm{E}-04$ & $2.18 \mathrm{E}-06$ & 4.95E-07 & $5.41 \mathrm{E}-05$ \\
\hline $\mathrm{x}_{4}$ & $*$ & $\mathrm{x}$ & $6.27 \mathrm{E}-03$ & $971 \mathrm{E}+00$ & $8.51 \mathrm{E}-04$ & 4.18E-02 & $3.06 \mathrm{E}-02$ & 4.63E-05 & $1.14 \mathrm{E}-05$ & 4.22E-03 \\
\hline$x_{5}$ & $*$ & $\mathrm{x}_{6}$ & 4.89E-04 & $-1.29 \mathrm{E}+00$ & $1.37 \mathrm{E}-04$ & $-1.94 \mathrm{E}-03$ & $-3.40 \mathrm{E}-03$ & $5.45 \mathrm{E}-06$ & $8.29 \mathrm{E}-06$ & $-1.04 \mathrm{E}-04$ \\
\hline $\mathrm{x}_{5}$ & * & $\mathrm{x}_{7}$ & $.32 \mathrm{E}-06$ & 4.93E-02 & $.66 \mathrm{E}-07$ & $.47 \mathrm{E}-05$ & $5.54 \mathrm{E}-05$ & $-1.56 \mathrm{E}-07$ & $.78 \mathrm{E}-10$ & $-9.36 \mathrm{E}-06$ \\
\hline$x_{5}$ & * & $\mathrm{x}_{8}$ & 4.97E-08 & $2.10 \mathrm{E}-02$ & $-9.68 \mathrm{E}-07$ & $-4.62 \mathrm{E}-05$ & $8.76 \mathrm{E}-05$ & $-4.88 \mathrm{E}-10$ & $-4.37 \mathrm{E}-08$ & $3.11 \mathrm{E}-06$ \\
\hline $\mathrm{x}_{5}$ & * & $\mathrm{x}_{9}$ & $.02 \mathrm{E}-04$ & $.00 \mathrm{E}+00$ & $.04 \mathrm{E}-04$ & $.53 \mathrm{E}-02$ & $2.12 \mathrm{E}-02$ & $5.43 \mathrm{E}-06$ & $2.19 \mathrm{E}-05$ & $-3.05 \mathrm{E}-03$ \\
\hline $\mathrm{X}_{5}$ & $*$ & $\mathrm{x}_{10}$ & $-2.89 \mathrm{E}-04$ & $-9.90 \mathrm{E}+00$ & $-3.23 \mathrm{E}-04$ & $-7.07 \mathrm{E}-03$ & $-1.32 \mathrm{E}-02$ & $-6.36 \mathrm{E}-05$ & $-3.30 \mathrm{E}-05$ & $-1.39 \mathrm{E}-03$ \\
\hline$x_{5}$ & * & $\mathrm{x}_{11}$ & $1.88 \mathrm{E}-05$ & $3.05 \mathrm{E}-02$ & $7.60 \mathrm{E}-06$ & $-4.04 \mathrm{E}-04$ & 7.06E-04 & $1.45 \mathrm{E}-06$ & $2.09 \mathrm{E}-07$ & $-4.08 \mathrm{E}-05$ \\
\hline $\mathrm{x}_{5}$ & * & $\mathrm{x}_{12}$ & $4.74 \mathrm{E}-05$ & $1.46 \mathrm{E}-01$ & $-1.46 \mathrm{E}-05$ & $-6.79 \mathrm{E}-04$ & $6.58 \mathrm{E}-04$ & $-3.35 \mathrm{E}-06$ & $3.17 \mathrm{E}-07$ & $1.64 \mathrm{E}-06$ \\
\hline $\mathrm{x}_{5}$ & * & $\mathrm{x}_{13}$ & $.23 \mathrm{E}-03$ & $-1.17 \mathrm{E}+00$ & $4.53 \mathrm{E}-05$ & $-5.73 \mathrm{E}-03$ & $1.36 \mathrm{E}-02$ & $-2.76 \mathrm{E}-05$ & $1.21 \mathrm{E}-05$ & $1.15 \mathrm{E}-03$ \\
\hline $\mathrm{x}_{5}$ & * & $\mathrm{x}_{14}$ & 1.69E-05 & $-1.13 \mathrm{E}-01$ & $8.20 \mathrm{E}-06$ & $1.87 \mathrm{E}-04$ & $1.22 \mathrm{E}-03$ & $2.61 \mathrm{E}-06$ & $-1.81 \mathrm{E}-07$ & $-5.59 \mathrm{E}-06$ \\
\hline $\mathrm{x}_{6}$ & * & $\mathrm{x}_{7}$ & $1.69 \mathrm{E}-02$ & $-5.74 \mathrm{E}+00$ & $6.87 \mathrm{E}-03$ & $1.65 \mathrm{E}-01$ & $1.35 \mathrm{E}-01$ & $3.42 \mathrm{E}-04$ & $4.54 \mathrm{E}-05$ & $1.28 \mathrm{E}-02$ \\
\hline $\mathrm{x}_{6}$ & * & $\mathrm{x}_{8}$ & $1.99 \mathrm{E}-03$ & $-5.13 \mathrm{E}-01$ & $1.12 \mathrm{E}-03$ & $3.30 \mathrm{E}-02$ & $1.01 \mathrm{E}-02$ & $8.22 \mathrm{E}-06$ & $-1.49 \mathrm{E}-06$ & $1.97 \mathrm{E}-03$ \\
\hline $\mathrm{x}_{6}$ & * & $\mathrm{X}_{9}$ & $7.28 \mathrm{E}-05$ & $-8.53 \mathrm{E}-02$ & $3.46 \mathrm{E}-05$ & $8.18 \mathrm{E}-04$ & $4.13 \mathrm{E}-04$ & 1.5 & 07 & E-05 \\
\hline $\mathrm{x}_{6}$ & * & $\mathrm{x}_{10}$ & $-6.24 \mathrm{E}-06$ & $1.89 \mathrm{E}-03$ & 7.57E-07 & $-1.22 \mathrm{E}-05$ & 8.64E-06 & $-2.21 \mathrm{E}-07$ & $-2.70 \mathrm{E}-08$ & $-3.41 \mathrm{E}-06$ \\
\hline $\mathrm{x}_{6}$ & * & $\mathrm{x}_{11}$ & $7.39 \mathrm{E}-04$ & $1.53 \mathrm{E}+00$ & $1.86 \mathrm{E}-04$ & $7.81 \mathrm{E}-03$ & 6.4 & 5.9 & $2.56 \mathrm{E}-06$ & 4.40E-04 \\
\hline $\mathrm{x}_{6}$ & * & $\mathrm{x}_{12}$ & $2.20 \mathrm{E}-03$ & $1.09 \mathrm{E}+00$ & $6.82 \mathrm{E}-04$ & $1.99 \mathrm{E}-02$ & $1.14 \mathrm{E}-02$ & $2.31 \mathrm{E}-05$ & $2.96 \mathrm{E}-06$ & $1.10 \mathrm{E}-03$ \\
\hline $\mathrm{x}_{6}$ & * & $\mathrm{x}_{13}$ & $.58 \mathrm{E}-04$ & $-9.10 \mathrm{E}-02$ & $5.80 \mathrm{E}-05$ & $1.49 \mathrm{E}-03$ & $9.16 \mathrm{E}-04$ & $2.93 \mathrm{E}-06$ & $2.81 \mathrm{E}-07$ & $1.26 \mathrm{E}-04$ \\
\hline $\mathrm{x}_{6}$ & * & $\mathrm{x}_{14}$ & $1.82 \mathrm{E}-03$ & $1.06 \mathrm{E}+00$ & $6.38 \mathrm{E}-04$ & $1.66 \mathrm{E}-02$ & 1.04E-02 & $2.33 \mathrm{E}-05$ & 4.40E-06 & $1.11 \mathrm{E}-03$ \\
\hline $\mathrm{x}_{7}$ & * & $\mathrm{x}_{8}$ & $6.60 \mathrm{E}-04$ & 7.36E-01 & 4.24E-05 & $-6.12 \mathrm{E}-03$ & $-6.35 \mathrm{E}-03$ & $5.74 \mathrm{E}-06$ & $-3.41 \mathrm{E}-06$ & $-5.51 \mathrm{E}-04$ \\
\hline $\mathrm{x}_{7}$ & * & $\mathrm{x}_{9}$ & 7.04E-04 & $-2.08 \mathrm{E}+00$ & $5.51 \mathrm{E}-04$ & $9.76 \mathrm{E}-03$ & $5.51 \mathrm{E}-03$ & 2.17E-05 & $3.90 \mathrm{E}-06$ & 7.98E-06 \\
\hline $\mathrm{x}_{7}$ & * & $\mathrm{x}_{10}$ & 3.20E-04 & $4.78 \mathrm{E}-02$ & $3.25 \mathrm{E}-05$ & $-1.90 \mathrm{E}-03$ & $-6.38 \mathrm{E}-04$ & $-8.05 \mathrm{E}-06$ & $-1.12 \mathrm{E}-06$ & $-1.63 \mathrm{E}-04$ \\
\hline $\mathrm{x}_{7}$ & * & $\mathrm{x}_{11}$ & $-1.27 \mathrm{E}-03$ & $-3.16 \mathrm{E}+00$ & $6.52 \mathrm{E}-05$ & $-1.91 \mathrm{E}-03$ & $-3.02 \mathrm{E}-02$ & $9.25 \mathrm{E}-06$ & $-1.65 \mathrm{E}-05$ & $1.83 \mathrm{E}-03$ \\
\hline $\mathrm{x}_{7}$ & * & $\mathrm{x}_{12}$ & 2.92E-04 & $1.24 \mathrm{E}+01$ & 2.02E-04 & $1.37 \mathrm{E}-03$ & $1.10 \mathrm{E}-02$ & 4.49E-05 & 8.86E-06 & $1.41 \mathrm{E}-03$ \\
\hline $\mathrm{x}_{7}$ & * & $\mathrm{x}_{13}$ & $2.45 \mathrm{E}-03$ & $-4.99 \mathrm{E}-01$ & $9.17 \mathrm{E}-04$ & $2.26 \mathrm{E}-02$ & $1.59 \mathrm{E}-02$ & $3.74 \mathrm{E}-05$ & $4.52 \mathrm{E}-06$ & $2.29 \mathrm{E}-03$ \\
\hline $\mathrm{x}_{7}$ & * & $\mathrm{x}_{14}$ & $-3.15 \mathrm{E}-04$ & $7.79 \mathrm{E}+00$ & $-1.01 \mathrm{E}-04$ & $5.36 \mathrm{E}-03$ & $-8.09 \mathrm{E}-02$ & $-3.79 \mathrm{E}-05$ & $-1.30 \mathrm{E}-05$ & $9.28 \mathrm{E}-04$ \\
\hline $\mathrm{x}_{8}$ & * & $\mathrm{x}_{9}$ & $3.04 \mathrm{E}-05$ & 1.37E-01 & $9.66 \mathrm{E}-05$ & $2.70 \mathrm{E}-03$ & $3.88 \mathrm{E}-04$ & $-3.48 \mathrm{E}-06$ & $-5.41 \mathrm{E}-07$ & $9.71 \mathrm{E}-05$ \\
\hline $\mathrm{x}_{8}$ & * & $\mathrm{x}_{10}$ & $.06 \mathrm{E}-05$ & 6.97E-02 & $-1.66 \mathrm{E}-07$ & $6.65 \mathrm{E}-04$ & $-8.17 \mathrm{E}-04$ & $-1.14 \mathrm{E}-07$ & $-5.50 \mathrm{E}-07$ & $-4.83 \mathrm{E}-05$ \\
\hline $\mathrm{X}_{8}$ & * & $\mathrm{x}_{11}$ & $-1.53 \mathrm{E}-03$ & $-4.15 \mathrm{E}+00$ & $-1.30 \mathrm{E}-03$ & $-7.31 \mathrm{E}-02$ & 7.48E-02 & $1.23 \mathrm{E}-04$ & 4.44E-05 & $8.01 \mathrm{E}-03$ \\
\hline $\mathrm{x}_{8}$ & * & $\mathrm{x}_{12}$ & 4.53E-04 & $7.87 \mathrm{E}+00$ & 1.1JL-04 & $-6.67 \mathrm{E}-03$ & (1.0) & $-6.60 \mathrm{E}$ & $1.77 \mathrm{E}$ & $-1.26 \mathrm{E}-02$ \\
\hline $\mathrm{x}_{8}$ & * & $\mathrm{x}_{13}$ & $5.07 \mathrm{E}-04$ & $-1.14 \mathrm{E}-01$ & $1.87 \mathrm{E}-04$ & 7.06E-03 & $-3.32 \mathrm{E}-04$ & $1.32 \mathrm{E}-06$ & $-2.16 \mathrm{E}-06$ & $5.45 \mathrm{E}-04$ \\
\hline $\mathrm{x}_{8}$ & 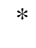 & $\mathrm{x}_{14}$ & $-1.15 \mathrm{E}-03$ & 等 & 4 & 3 & 3 & 4 & 06 & 03 \\
\hline $\mathrm{x}_{9}$ & $*$ & $\mathrm{x}_{10}$ & $-8.55 \mathrm{E}-07$ & $-1.57 \mathrm{E}-03$ & $7.50 \mathrm{E}-08$ & $-1.04 \mathrm{E}-05$ & 1.07E-05 & $1.18 \mathrm{E}-08$ & 5.17E-09 & $3.40 \mathrm{E}-07$ \\
\hline $\mathrm{x}_{9}$ & * & $\mathrm{x}_{11}$ & $3.03 \mathrm{E}-05$ & $-1.77 \mathrm{E}-01$ & $160^{2}$ & 4.47E-04 & $-4.05 \mathrm{E}-04$ & $1.23 \mathrm{E}-06$ & $-1.38 \mathrm{E}-07$ & $-3.68 \mathrm{E}-05$ \\
\hline $\mathrm{x}_{9}$ & * & $\mathrm{x}_{12}$ & $1.60 \mathrm{E}-04$ & $-5.06 \mathrm{E}-02$ & $5.37 \mathrm{E}-05$ & $1.79 \mathrm{E}-03$ & $3.60 \mathrm{E}-$ & $1.84 \mathrm{E}-06$ & $4.56 \mathrm{E}-08$ & $8.02 \mathrm{E}-05$ \\
\hline $\mathrm{x}_{9}$ & 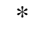 & $\mathrm{x}_{13}$ & . & -1.201 & (5. & 05 & 0.03 & 2.00 & 08 & 4.06 \\
\hline $\mathrm{x}_{9}$ & * & $\mathrm{x}_{14}$ & $.21 \mathrm{E}-04$ & $-1.01 \mathrm{E}-01$ & $5.36 \mathrm{E}-05$ & $1.37 \mathrm{E}-03$ & $5.86 \mathrm{E}-04$ & $2.15 \mathrm{E}-06$ & 2.91E-07 & $8.38 \mathrm{E}-05$ \\
\hline $\mathrm{x}_{10}$ & $*$ & $\mathrm{x}_{11}$ & $-2.01 \mathrm{~L}-$ & $5 \mathrm{E}-03$ & $-4.83 \mathrm{E}-07$ & -1. & 3 & 7 & 08 & -6 \\
\hline $\mathrm{x}_{10}$ & * & $\mathrm{x}_{12}$ & $-3.62 E-05$ & $-2.15 \mathrm{E}-02$ & $-3.46 \mathrm{E}-06$ & $-2.21 \mathrm{E}-04$ & $-1.53 \mathrm{E}-04$ & $-7.32 \mathrm{E}-07$ & $-1.85 \mathrm{E}-07$ & $-1.23 \mathrm{E}-05$ \\
\hline $\mathrm{x}_{10}$ & * & $\mathrm{x}_{13}$ & $-1.54 \mathrm{E}-06$ & 4.95E-04 & $-1.95 \mathrm{E}-07$ & $-3.27 \mathrm{E}-06$ & $-1.39 \mathrm{E}-05$ & $-4.30 \mathrm{E}$ & $-9.83 \mathrm{E}-09$ & $-1.20 \mathrm{E}-06$ \\
\hline $\mathrm{x}_{10}$ & $*$ & $\mathrm{x}_{14}$ & $-3.97 \mathrm{E}-05$ & $-2.35 \mathrm{E}-02$ & $-3.98 \mathrm{E}-06$ & $-2.56 \mathrm{E}-04$ & $-1.44 \mathrm{E}$ & $-7.07 \mathrm{E}-07$ & $-1.75 \mathrm{E}-07$ & $-1.14 \mathrm{E}-05$ \\
\hline $\mathrm{x}_{11}$ & * & $\mathrm{x}_{12}$ & $1.01 \mathrm{E}-03$ & $1.12 \mathrm{E}+01$ & $2.95 \mathrm{E}-04$ & $2.77 \mathrm{E}-02$ & $1.92 \mathrm{E}-02$ & $-4.02 \mathrm{E}-05$ & $1.20 \mathrm{E}-05$ & $-1.12 \mathrm{E}-03$ \\
\hline $\mathrm{x}_{11}$ & * & $\mathrm{x}_{13}$ & 15 & 3.04 & $2.84 \mathrm{E}-05$ & 4 & 9. & 1.14 & 4.37E-07 & $1.04 \mathrm{E}-04$ \\
\hline $\mathrm{x}_{11}$ & * & $\mathrm{x}_{14}$ & $3.32 \mathrm{E}-04$ & $9.95 \mathrm{E}+00$ & $4.56 \mathrm{E}-04$ & $1.93 \mathrm{E}-02$ & $2.57 \mathrm{E}-02$ & $-2.85 \mathrm{E}-05$ & 1.64E-05 & $-3.32 \mathrm{E}-04$ \\
\hline$x_{12}$ & $*$ & $\mathrm{x}_{13}$ & E-04 & $-3.07 \mathrm{E}-01$ & E-05 & $1.94 \mathrm{E}-03$ & $1.91 \mathrm{E}-06$ & $2.95 \mathrm{E}-06$ & $-3.91 \mathrm{E}-07$ & $1.63 \mathrm{E}-04$ \\
\hline $\mathrm{x}_{12}$ & * & $\mathrm{x}_{14}$ & $1.26 \mathrm{E}-03$ & $6.25 \mathrm{E}+00$ & $2.02 \mathrm{E}-04$ & 7.70E-04 & $-1.36 \mathrm{E}-02$ & $-4.75 \mathrm{E}-05$ & $-1.21 \mathrm{E}-05$ & $-5.09 \mathrm{E}-04$ \\
\hline
\end{tabular}


quality

\begin{tabular}{|c|c|c|c|c|c|c|c|c|c|}
\hline \multirow[t]{3}{*}{$\mathrm{x}_{13}$} & * $\quad \mathrm{x}_{14}$ & $1.40 \mathrm{E}-04$ & $-3.67 \mathrm{E}-01$ & $5.52 \mathrm{E}-05$ & $1.22 \mathrm{E}-03$ & $2.54 \mathrm{E}-04$ & $3.98 \mathrm{E}-06$ & 5.89E-08 & $1.90 \mathrm{E}-04$ \\
\hline & $\mathrm{c}$ & $2.45 \mathrm{E}-05$ & $-1.11 \mathrm{E}-03$ & $8.36 \mathrm{E}-06$ & $2.42 \mathrm{E}-04$ & $1.24 \mathrm{E}-04$ & $3.82 \mathrm{E}-07$ & $1.50 \mathrm{E}-08$ & $1.72 \mathrm{E}-05$ \\
\hline & $r^{2}$ & 0.631 & 0.615 & 0.28 & 0.462 & 0.431 & 0.884 & 0.549 & 0.738 \\
\hline
\end{tabular}

Table 6. The $\mathrm{x}$ and $\mathrm{y}$ variables, their coefficient and determination coefficient of equations belonging non-linear models yielded from the data collected Station 3

\begin{tabular}{|c|c|c|c|c|c|c|c|c|c|c|}
\hline & & & $\mathrm{y}_{1}$ & $\mathrm{y}_{2}$ & $\mathrm{y}_{3}$ & $\mathrm{y}_{4}$ & $\mathrm{y}_{5}$ & $\mathrm{y}_{6}$ & $\mathrm{y}_{7}$ & $\mathrm{y}_{8}$ \\
\hline & $\mathrm{x}_{1}$ & & $-9.25 \mathrm{E}-02$ & $-9.72 \mathrm{E}-02$ & $6.99 \mathrm{E}-04$ & $-7.34 \mathrm{E}-03$ & $-2.17 \mathrm{E}+00$ & 3.63E-04 & $4.11 \mathrm{E}-04$ & $-1.44 \mathrm{E}-03$ \\
\hline & $\mathrm{x}_{2}$ & & $-1.68 \mathrm{E}-02$ & $-1.11 \mathrm{E}-01$ & $1.02 \mathrm{E}-05$ & $-1.51 \mathrm{E}-01$ & 8.03E-01 & $6.62 \mathrm{E}-04$ & $-3.99 \mathrm{E}-05$ & $-8.80 \mathrm{E}-05$ \\
\hline & $\mathrm{x}_{3}$ & & $-8.95 \mathrm{E}-02$ & $-1.90 \mathrm{E}-01$ & $1.40 \mathrm{E}-03$ & $-3.51 \mathrm{E}-01$ & $-3.20 \mathrm{E}-01$ & $1.83 \mathrm{E}-03$ & $2.76 \mathrm{E}-04$ & $-5.30 \mathrm{E}-05$ \\
\hline & $\mathrm{x}_{4}$ & & $7.57 \mathrm{E}-03$ & $4.91 \mathrm{E}-01$ & $-1.08 \mathrm{E}-03$ & 8.25E-01 & $-4.29 \mathrm{E}+00$ & $-1.82 \mathrm{E}-03$ & $-1.39 \mathrm{E}-04$ & $-2.76 \mathrm{E}-03$ \\
\hline & $\mathrm{x}_{5}$ & & $3.54 \mathrm{E}-01$ & $2.29 \mathrm{E}-01$ & $2.17 \mathrm{E}-02$ & $-2.10 \mathrm{E}-01$ & $9.08 \mathrm{E}+00$ & $-6.64 E-03$ & $5.68 \mathrm{E}-04$ & $-4.20 \mathrm{E}-03$ \\
\hline & $\mathrm{x}_{6}$ & & $-3.27 \mathrm{E}-02$ & $-2.90 \mathrm{E}-01$ & $1.25 \mathrm{E}-03$ & $-3.64 \mathrm{E}-01$ & $1.79 \mathrm{E}+00$ & $6.83 \mathrm{E}-04$ & $1.33 \mathrm{E}-04$ & $-7.51 \mathrm{E}-04$ \\
\hline & $\mathrm{x}_{7}$ & & $6.70 \mathrm{E}-02$ & $-1.64 \mathrm{E}+00$ & $1.07 \mathrm{E}-02$ & $-1.68 \mathrm{E}+00$ & $1.70 \mathrm{E}+01$ & 4.17E-03 & $2.05 \mathrm{E}-04$ & $-1.49 \mathrm{E}-02$ \\
\hline & $\mathrm{x}_{8}$ & & $-1.39 \mathrm{E}-01$ & $-3.90 \mathrm{E}-01$ & $-3.79 \mathrm{E}-03$ & $-1.26 \mathrm{E}-01$ & $5.56 \mathrm{E}-01$ & $3.64 \mathrm{E}-03$ & $-2.64 \mathrm{E}-04$ & $-6.33 \mathrm{E}-03$ \\
\hline & $\mathrm{x}_{9}$ & & $-7.31 \mathrm{E}-03$ & $-6.42 \mathrm{E}-02$ & $1.44 \mathrm{E}-04$ & $-7.39 \mathrm{E}-02$ & 4.07E-01 & $1.31 \mathrm{E}-04$ & $1.59 \mathrm{E}-05$ & $-1.49 \mathrm{E}-04$ \\
\hline & $\mathrm{x}_{10}$ & & $-1.78 \mathrm{E}-03$ & $-4.52 \mathrm{E}-03$ & $4.30 \mathrm{E}-05$ & $-3.71 \mathrm{E}-03$ & $-4.22 \mathrm{E}-02$ & $8.50 \mathrm{E}-06$ & $1.01 \mathrm{E}-05$ & $-2.80 \mathrm{E}-05$ \\
\hline & $\mathrm{x}_{11}$ & & $1.55 \mathrm{E}-02$ & $1.55 \mathrm{E}-01$ & $1.06 \mathrm{E}-04$ & $1.95 \mathrm{E}-01$ & $-1.33 \mathrm{E}+00$ & $-7.64 \mathrm{E}-04$ & $1.01 \mathrm{E}-04$ & 5.43E-05 \\
\hline & $\mathrm{x}_{12}$ & & $2.41 \mathrm{E}-02$ & 8.36E-01 & $3.88 \mathrm{E}-03$ & $5.78 \mathrm{E}-01$ & $-5.72 \mathrm{E}+00$ & $-1.45 \mathrm{E}-03$ & $-6.59 \mathrm{E}-05$ & $5.45 \mathrm{E}-03$ \\
\hline & $x_{13}$ & & $-2.32 \mathrm{E}-02$ & $-1.53 \mathrm{E}-01$ & $2.10 \mathrm{E}-07$ & $-1.33 \mathrm{E}-01$ & $6.39 \mathrm{E}-01$ & $3.11 \mathrm{E}-04$ & 4.34E-05 & $-6.12 \mathrm{E}-04$ \\
\hline & $\mathrm{x}_{14}$ & & $-6.31 \mathrm{E}-02$ & $-4.62 \mathrm{E}-02$ & $1.63 \mathrm{E}-03$ & $-1.78 \mathrm{E}-01$ & $-6.89 \mathrm{E}-01$ & $1.28 \mathrm{E}-03$ & $5.03 \mathrm{E}-05$ & $1.20 \mathrm{E}-03$ \\
\hline $\mathrm{x}_{1}$ & $*$ & $\mathrm{x}_{1}$ & $1.61 \mathrm{E}-02$ & $-5.96 \mathrm{E}-01$ & $2.33 \mathrm{E}-03$ & $-8.01 \mathrm{E}-01$ & $4.77 \mathrm{E}+00$ & $1.27 \mathrm{E}-03$ & $-5.23 \mathrm{E}-04$ & $-2.49 \mathrm{E}-04$ \\
\hline $\mathrm{x}_{2}$ & $*$ & $\mathrm{x}_{2}$ & $-5.16 \mathrm{E}-03$ & $-3.13 \mathrm{E}-02$ & 2.13E-03 & 4.03E-02 & $-1.20 \mathrm{E}+00$ & $-2.02 \mathrm{E}-03$ & $5.53 \mathrm{E}-04$ & $2.30 \mathrm{E}-04$ \\
\hline $\mathrm{x}_{3}$ & $*$ & $\mathrm{x}_{3}$ & $1.32 \mathrm{E}+00$ & $5.01 \mathrm{E}+00$ & $1.10 \mathrm{E}-02$ & $4.80 \mathrm{E}+00$ & $-1.40 \mathrm{E}+01$ & $-2.18 \mathrm{E}-02$ & 4.37E-04 & $1.44 \mathrm{E}-02$ \\
\hline $\mathrm{x}_{4}$ & $*$ & $\mathrm{x}_{4}$ & $-7.43 \mathrm{E}-02$ & $-1.86 \mathrm{E}-01$ & $-6.30 \mathrm{E}-04$ & $-3.84 \mathrm{E}-01$ & $1.92 \mathrm{E}+00$ & $3.75 \mathrm{E}-03$ & $-4.79 \mathrm{E}-04$ & $-1.65 \mathrm{E}-03$ \\
\hline $\mathrm{x}_{5}$ & $*$ & $\mathrm{x}_{5}$ & $5.19 \mathrm{E}-04$ & $3.53 \mathrm{E}-02$ & $-6.10 \mathrm{E}-05$ & 2.09E-02 & $-2.50 \mathrm{E}-01$ & $2.53 \mathrm{E}-04$ & $-1.41 \mathrm{E}-05$ & $1.39 \mathrm{E}-04$ \\
\hline $\mathrm{x}_{6}$ & $*$ & $\mathrm{x}_{6}$ & $-2.30 \mathrm{E}-01$ & $-1.56 \mathrm{E}+00$ & $4.75 \mathrm{E}-03$ & $-2.29 \mathrm{E}+00$ & $9.65 \mathrm{E}+00$ & $7.20 \mathrm{E}-03$ & $3.69 \mathrm{E}-04$ & $-2.08 \mathrm{E}-03$ \\
\hline$x_{7}$ & $*$ & $\mathrm{x}_{7}$ & $-1.14 \mathrm{E}-02$ & $-3.71 \mathrm{E}-02$ & $5.94 \mathrm{E}-05$ & $-1.01 \mathrm{E}-02$ & $-3.31 \mathrm{E}-02$ & $-1.74 \mathrm{E}-04$ & $2.95 \mathrm{E}-05$ & $-1.74 \mathrm{E}-04$ \\
\hline $\mathrm{x}_{8}$ & $*$ & $\mathrm{x}_{8}$ & $-1.66 \mathrm{E}-04$ & $1.88 \mathrm{E}-03$ & 7.42E-06 & $2.17 \mathrm{E}-03$ & $-1.55 \mathrm{E}-02$ & $1.49 \mathrm{E}-06$ & $-4.24 \mathrm{E}-06$ & 2.19E-05 \\
\hline $\mathrm{x}_{9}$ & $*$ & $\mathrm{x}_{9}$ & $4.40 \mathrm{E}-02$ & $-1.60 \mathrm{E}-01$ & $-2.54 \mathrm{E}-04$ & $-8.42 \mathrm{E}-02$ & $2.71 \mathrm{E}+00$ & $-1.80 \mathrm{E}-03$ & $-1.59 \mathrm{E}-04$ & $-7.41 \mathrm{E}-04$ \\
\hline $\mathrm{x}_{10}$ & $*$ & $\mathrm{x}_{10}$ & $-2.14 \mathrm{E}-12$ & $-1.66 \mathrm{E}-11$ & $-4.40 \mathrm{E}-15$ & $-1.15 \mathrm{E}-11$ & $6.82 \mathrm{E}-11$ & $4.88 \mathrm{E}-15$ & $5.04 \mathrm{E}-15$ & $-7.99 \mathrm{E}-14$ \\
\hline $\mathrm{x}_{11}$ & $*$ & $\mathrm{x}_{11}$ & $-1.04 \mathrm{E}-01$ & $3.47 \mathrm{E}-01$ & $-3.65 \mathrm{E}-03$ & $3.47 \mathrm{E}-01$ & $-5.51 \mathrm{E}+00$ & $5.27 \mathrm{E}-03$ & $-2.19 \mathrm{E}-04$ & $-2.33 \mathrm{E}-03$ \\
\hline$x_{12}$ & $*$ & $\mathrm{x}_{12}$ & $2.63 \mathrm{E}-02$ & $-4.74 \mathrm{E}-01$ & $-4.86 \mathrm{E}-04$ & $-6.64 \mathrm{E}-01$ & $2.92 \mathrm{E}+00$ & $8.18 \mathrm{E}-03$ & $-6.12 \mathrm{E}-04$ & $2.86 \mathrm{E}-03$ \\
\hline $\mathrm{x}_{13}$ & $*$ & $\mathrm{x}_{13}$ & $-7.60 \mathrm{E}-02$ & $-4.52 \mathrm{E}-01$ & $6.99 \mathrm{E}-04$ & $-3.65 \mathrm{E}-01$ & $1.48 \mathrm{E}+00$ & $1.73 \mathrm{E}-04$ & $2.71 \mathrm{E}-04$ & $-2.27 \mathrm{E}-03$ \\
\hline $\mathrm{x}_{14}$ & $*$ & $\mathrm{x}_{14}$ & $5.02 \mathrm{E}-01$ & $7.79 \mathrm{E}+00$ & $2.85 \mathrm{E}-04$ & $5.51 \mathrm{E}+00$ & $-6.26 \mathrm{E}+01$ & $-6.75 \mathrm{E}-03$ & $2.99 \mathrm{E}-03$ & $2.16 \mathrm{E}-02$ \\
\hline $\mathrm{x}_{1}$ & $*$ & $\mathrm{x}_{2}$ & 4.95E-01 & $1.46 \mathrm{E}+00$ & $-2.15 \mathrm{E}-02$ & $2.95 \mathrm{E}+00$ & $-8.61 \mathrm{E}+00$ & $-1.04 \mathrm{E}-03$ & $-1.33 \mathrm{E}-03$ & $-2.46 \mathrm{E}-02$ \\
\hline $\mathrm{x}_{1}$ & $*$ & $\mathrm{x}_{3}$ & $-2.70 \mathrm{E}-01$ & $1.57 \mathrm{E}+00$ & $6.81 \mathrm{E}-03$ & $1.76 \mathrm{E}+00$ & $-1.70 \mathrm{E}+01$ & 7.31E-04 & $3.08 \mathrm{E}-04$ & $-2.93 \mathrm{E}-03$ \\
\hline $\mathrm{x}_{1}$ & $*$ & $\mathrm{x}_{4}$ & $1.62 \mathrm{E}-02$ & $-1.97 \mathrm{E}-01$ & $-2.51 \mathrm{E}-03$ & $3.38 \mathrm{E}-01$ & $8.83 \mathrm{E}-01$ & $-2.03 \mathrm{E}-04$ & $-5.84 \mathrm{E}-04$ & $1.54 \mathrm{E}-03$ \\
\hline $\mathrm{x}_{1}$ & $*$ & $\mathrm{x}_{5}$ & $6.89 \mathrm{E}-02$ & $1.06 \mathrm{E}-01$ & $-6.15 \mathrm{E}-04$ & $5.16 \mathrm{E}-02$ & $-1.38 \mathrm{E}-02$ & $-1.61 \mathrm{E}-04$ & $-1.09 \mathrm{E}-05$ & $1.63 \mathrm{E}-03$ \\
\hline $\mathrm{x}_{1}$ & $*$ & $\mathrm{x}_{6}$ & $3.98 \mathrm{E}-01$ & $1.90 \mathrm{E}+00$ & $-1.99 \mathrm{E}-02$ & $2.33 \mathrm{E}+00$ & $-1.71 \mathrm{E}+00$ & $-4.61 \mathrm{E}-03$ & $-1.84 \mathrm{E}-03$ & 7.49E-03 \\
\hline $\mathrm{x}_{1}$ & $*$ & $\mathrm{x}_{7}$ & $-8.49 \mathrm{E}-02$ & $-2.90 \mathrm{E}-01$ & $1.13 \mathrm{E}-03$ & $-2.09 \mathrm{E}-01$ & $1.12 \mathrm{E}+00$ & $-2.79 \mathrm{E}-04$ & $1.82 \mathrm{E}-04$ & $-2.66 \mathrm{E}-03$ \\
\hline $\mathrm{x}_{1}$ & $*$ & $\mathrm{x}_{8}$ & $1.61 \mathrm{E}-01$ & $-2.50 \mathrm{E}-01$ & $2.86 \mathrm{E}-03$ & $-5.33 \mathrm{E}-02$ & $9.52 \mathrm{E}-01$ & $-4.23 \mathrm{E}-03$ & $1.90 \mathrm{E}-03$ & 4.48E-04 \\
\hline $\mathrm{x}_{1}$ & $*$ & $\mathrm{x}_{9}$ & $-4.33 \mathrm{E}-02$ & $8.75 \mathrm{E}-01$ & $1.05 \mathrm{E}-02$ & $2.62 \mathrm{E}+00$ & $-2.92 \mathrm{E}+01$ & $-1.95 \mathrm{E}-02$ & $6.10 \mathrm{E}-03$ & $-8.63 \mathrm{E}-03$ \\
\hline $\mathrm{x}_{1}$ & $*$ & $\mathrm{x}_{10}$ & $-3.82 \mathrm{E}-02$ & $-6.60 \mathrm{E}-02$ & $3.86 \mathrm{E}-04$ & $-2.27 \mathrm{E}-01$ & $-8.65 \mathrm{E}-01$ & $2.18 \mathrm{E}-03$ & $1.77 \mathrm{E}-04$ & $-1.98 \mathrm{E}-04$ \\
\hline $\mathrm{x}_{1}$ & $*$ & $\mathrm{x}_{11}$ & $-7.42 \mathrm{E}-01$ & $-3.50 \mathrm{E}+00$ & $8.04 \mathrm{E}-03$ & $-3.92 \mathrm{E}+00$ & $1.26 \mathrm{E}+01$ & $5.95 \mathrm{E}-03$ & $-1.60 \mathrm{E}-03$ & $-2.85 \mathrm{E}-02$ \\
\hline $\mathrm{x}_{1}$ & $*$ & $\mathrm{x}_{12}$ & $3.10 \mathrm{E}-01$ & $1.65 \mathrm{E}+00$ & $-1.01 \mathrm{E}-02$ & $9.55 \mathrm{E}-02$ & 6.17E-01 & 7.31E-03 & $-2.21 \mathrm{E}-03$ & $3.97 \mathrm{E}-03$ \\
\hline
\end{tabular}




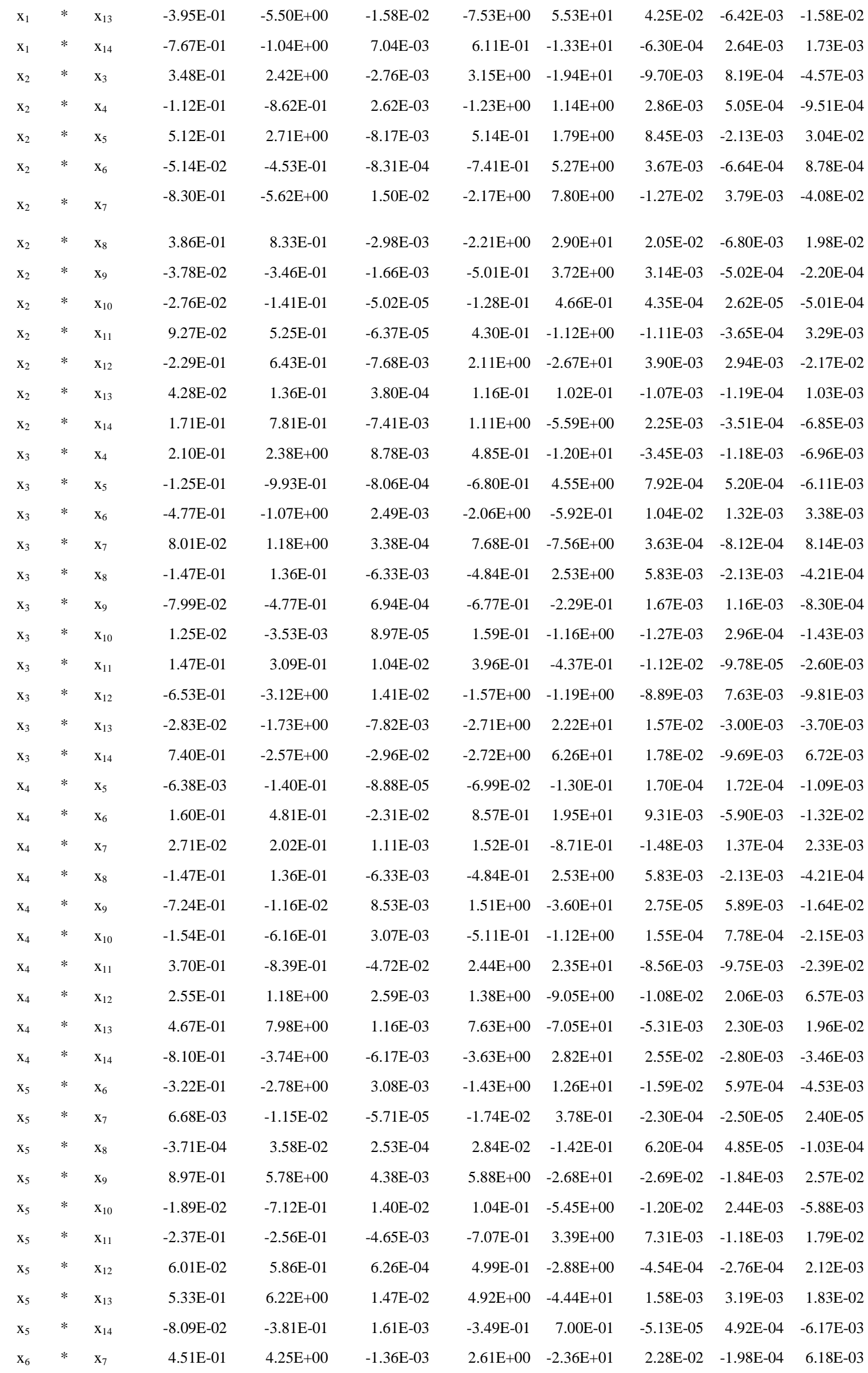


quality

\begin{tabular}{|c|c|c|c|c|c|c|c|c|c|c|}
\hline $\mathrm{x}_{6}$ & $*$ & $\mathrm{X}_{8}$ & $1.30 \mathrm{E}-01$ & $1.06 \mathrm{E}+00$ & $2.41 \mathrm{E}-03$ & $1.01 \mathrm{E}-01$ & $-1.59 \mathrm{E}+00$ & $5.97 \mathrm{E}-03$ & $1.48 \mathrm{E}-03$ & $-4.44 \mathrm{E}-03$ \\
\hline $\mathrm{x}_{6}$ & $*$ & $\mathrm{X}_{9}$ & $-1.11 \mathrm{E}-01$ & $-6.82 \mathrm{E}-01$ & $1.39 \mathrm{E}-03$ & $-6.87 \mathrm{E}-01$ & $2.66 \mathrm{E}+00$ & $1.05 \mathrm{E}-03$ & 4.42E-04 & $-2.80 \mathrm{E}-03$ \\
\hline $\mathrm{x}_{6}$ & $*$ & $\mathrm{x}_{10}$ & $-3.37 \mathrm{E}-02$ & $-2.72 \mathrm{E}-01$ & $8.18 \mathrm{E}-06$ & $-4.23 \mathrm{E}-01$ & $2.81 \mathrm{E}+00$ & 2.03E-03 & $-2.60 \mathrm{E}-04$ & $8.12 \mathrm{E}-04$ \\
\hline $\mathrm{x}_{6}$ & $*$ & $\mathrm{x}_{11}$ & $2.37 \mathrm{E}-01$ & $1.67 \mathrm{E}+00$ & $-3.01 \mathrm{E}-03$ & $1.74 \mathrm{E}+00$ & $-8.41 \mathrm{E}+00$ & $-3.97 \mathrm{E}-03$ & $-3.40 \mathrm{E}-04$ & $4.01 \mathrm{E}-03$ \\
\hline $\mathrm{x}_{6}$ & $*$ & $\mathrm{x}_{12}$ & $2.65 \mathrm{E}-01$ & $3.44 \mathrm{E}+00$ & $3.66 \mathrm{E}-03$ & $1.49 \mathrm{E}+00$ & $-5.56 \mathrm{E}+00$ & $-2.82 \mathrm{E}-03$ & $-4.69 \mathrm{E}-03$ & $3.59 \mathrm{E}-02$ \\
\hline $\mathrm{x}_{6}$ & $*$ & $\mathrm{x}_{13}$ & $-1.50 \mathrm{E}-01$ & $-1.02 \mathrm{E}+00$ & $-3.08 \mathrm{E}-04$ & $-9.98 \mathrm{E}-01$ & $5.14 \mathrm{E}+00$ & $3.00 \mathrm{E}-03$ & $1.36 \mathrm{E}-04$ & $-2.50 \mathrm{E}-03$ \\
\hline $\mathrm{x}_{6}$ & $*$ & $\mathrm{x}_{14}$ & $-5.03 \mathrm{E}-01$ & $-8.57 \mathrm{E}-01$ & $-2.64 \mathrm{E}-03$ & $-1.67 \mathrm{E}+00$ & $2.23 \mathrm{E}+00$ & $1.48 \mathrm{E}-02$ & $-1.76 \mathrm{E}-03$ & $8.75 \mathrm{E}-03$ \\
\hline $\mathrm{x}_{7}$ & $*$ & $\mathrm{X}_{8}$ & $-8.01 \mathrm{E}-03$ & $-4.91 \mathrm{E}-02$ & $-3.12 \mathrm{E}-04$ & $-2.06 \mathrm{E}-02$ & $2.87 \mathrm{E}-02$ & $-8.97 \mathrm{E}-04$ & $-1.78 \mathrm{E}-04$ & $4.70 \mathrm{E}-04$ \\
\hline $\mathrm{x}_{7}$ & $*$ & $\mathrm{x}_{9}$ & $-1.41 \mathrm{E}+00$ & $-9.58 \mathrm{E}+00$ & $-8.75 \mathrm{E}-03$ & $-1.01 \mathrm{E}+01$ & $4.99 \mathrm{E}+01$ & 4.92E-02 & $2.02 \mathrm{E}-03$ & $-4.37 \mathrm{E}-02$ \\
\hline $\mathrm{x}_{7}$ & $*$ & $\mathrm{x}_{10}$ & $-1.13 \mathrm{E}-01$ & $3.39 \mathrm{E}-01$ & $-2.33 \mathrm{E}-03$ & $-6.51 \mathrm{E}-01$ & 9.37E-01 & $1.28 \mathrm{E}-02$ & $-2.77 \mathrm{E}-03$ & $6.56 \mathrm{E}-03$ \\
\hline $\mathrm{x}_{7}$ & $*$ & $\mathrm{x}_{11}$ & 3.93E-01 & $4.50 \mathrm{E}-01$ & $5.98 \mathrm{E}-03$ & $1.00 \mathrm{E}+00$ & $-6.30 \mathrm{E}+00$ & $-1.02 \mathrm{E}-02$ & $2.77 \mathrm{E}-03$ & $-2.05 \mathrm{E}-02$ \\
\hline $\mathrm{x}_{7}$ & $*$ & $\mathrm{x}_{12}$ & $-6.90 \mathrm{E}-02$ & $-7.58 \mathrm{E}-01$ & $-1.37 \mathrm{E}-03$ & $-5.93 \mathrm{E}-01$ & $3.46 \mathrm{E}+00$ & 4.31E-04 & $4.86 \mathrm{E}-04$ & $-3.99 \mathrm{E}-03$ \\
\hline $\mathrm{x}_{7}$ & $*$ & $\mathrm{x}_{13}$ & $-6.76 \mathrm{E}-01$ & $-8.34 \mathrm{E}+00$ & $-1.65 \mathrm{E}-02$ & $-5.66 \mathrm{E}+00$ & $5.85 \mathrm{E}+01$ & $-1.71 \mathrm{E}-02$ & $-3.48 \mathrm{E}-03$ & $-2.13 \mathrm{E}-02$ \\
\hline $\mathrm{x}_{7}$ & $*$ & $\mathrm{x}_{14}$ & $1.44 \mathrm{E}-01$ & $2.86 \mathrm{E}-01$ & $-1.32 \mathrm{E}-03$ & $2.46 \mathrm{E}-01$ & $1.49 \mathrm{E}+00$ & 3.79E-04 & $-5.99 \mathrm{E}-04$ & $6.62 \mathrm{E}-03$ \\
\hline $\mathrm{x}_{8}$ & $*$ & $\mathrm{x}_{9}$ & $5.56 \mathrm{E}-01$ & $6.09 \mathrm{E}+00$ & $-1.95 \mathrm{E}-02$ & $3.63 \mathrm{E}+00$ & $-1.62 \mathrm{E}+01$ & $2.05 \mathrm{E}-02$ & $-7.45 \mathrm{E}-03$ & $2.94 \mathrm{E}-02$ \\
\hline $\mathrm{x}_{8}$ & $*$ & $\mathrm{x}_{10}$ & $-7.65 \mathrm{E}-02$ & $-8.26 \mathrm{E}-01$ & $2.54 \mathrm{E}-03$ & $-5.99 \mathrm{E}-01$ & $3.60 \mathrm{E}+00$ & $-1.31 \mathrm{E}-03$ & $7.55 \mathrm{E}-04$ & $-3.66 \mathrm{E}-03$ \\
\hline $\mathrm{x}_{8}$ & $*$ & $\mathrm{x}_{11}$ & $-1.71 \mathrm{E}-01$ & $-5.17 \mathrm{E}+00$ & $-2.62 \mathrm{E}-03$ & $-2.46 \mathrm{E}+00$ & $3.83 \mathrm{E}+01$ & $1.66 \mathrm{E}-03$ & $1.53 \mathrm{E}-03$ & $-4.56 \mathrm{E}-02$ \\
\hline $\mathrm{x}_{8}$ & $*$ & $\mathrm{x}_{12}$ & $-1.46 \mathrm{E}-01$ & $1.31 \mathrm{E}-01$ & $-3.36 \mathrm{E}-03$ & $-1.97 \mathrm{E}-01$ & $1.08 \mathrm{E}+00$ & $6.95 \mathrm{E}-03$ & $-1.59 \mathrm{E}-03$ & $-2.91 \mathrm{E}-03$ \\
\hline $\mathrm{x}_{8}$ & $*$ & $\mathrm{x}_{13}$ & $-1.30 \mathrm{E}-01$ & $-2.33 \mathrm{E}+00$ & $3.56 \mathrm{E}-03$ & $-2.80 \mathrm{E}+00$ & $1.19 \mathrm{E}+01$ & $1.03 \mathrm{E}-02$ & $3.05 \mathrm{E}-03$ & $-1.21 \mathrm{E}-02$ \\
\hline $\mathrm{x}_{8}$ & $*$ & $\mathrm{x}_{14}$ & $2.91 \mathrm{E}-01$ & $-6.73 \mathrm{E}-01$ & $1.13 \mathrm{E}-02$ & $1.11 \mathrm{E}+00$ & $-7.28 \mathrm{E}+00$ & $-2.16 \mathrm{E}-02$ & 4.69E-03 & $3.02 \mathrm{E}-03$ \\
\hline $\mathrm{x}_{9}$ & $*$ & $\mathrm{x}_{10}$ & $0.00 \mathrm{E}+00$ & $0.00 \mathrm{E}+00$ & $0.00 \mathrm{E}+00$ & $0.00 \mathrm{E}+00$ & $0.00 \mathrm{E}+00$ & $0.00 \mathrm{E}+00$ & $0.00 \mathrm{E}+00$ & $0.00 \mathrm{E}+00$ \\
\hline $\mathrm{X}_{9}$ & $*$ & $\mathrm{x}_{11}$ & $8.74 \mathrm{E}-02$ & $1.56 \mathrm{E}-01$ & $-3.42 \mathrm{E}-03$ & $1.08 \mathrm{E}-01$ & $3.09 \mathrm{E}+00$ & $1.28 \mathrm{E}-03$ & $-9.42 \mathrm{E}-04$ & $9.86 \mathrm{E}-04$ \\
\hline $\mathrm{x}_{9}$ & $*$ & $\mathrm{x}_{12}$ & $1.70 \mathrm{E}-01$ & $1.77 \mathrm{E}+00$ & 7.73E-03 & $1.19 \mathrm{E}+00$ & $-2.00 \mathrm{E}+01$ & $1.05 \mathrm{E}-03$ & 2.64E-03 & $1.50 \mathrm{E}-02$ \\
\hline $\mathrm{X}_{9}$ & $*$ & $\mathrm{x}_{13}$ & 2.77E-02 & $1.05 \mathrm{E}-01$ & $-9.91 \mathrm{E}-04$ & $6.92 \mathrm{E}-02$ & 4.90E-01 & 2.64E-04 & $-1.65 \mathrm{E}-04$ & $6.18 \mathrm{E}-04$ \\
\hline $\mathrm{X}_{9}$ & $*$ & $\mathrm{x}_{14}$ & 2.19E-01 & $1.44 \mathrm{E}+00$ & $-9.89 \mathrm{E}-04$ & 7.47E-01 & $-5.51 \mathrm{E}+00$ & $3.36 \mathrm{E}-03$ & $-6.85 \mathrm{E}-04$ & $1.17 \mathrm{E}-02$ \\
\hline $\mathrm{x}_{10}$ & $*$ & $\mathrm{x}_{11}$ & $-7.11 \mathrm{E}-02$ & $-1.94 \mathrm{E}-01$ & $5.76 \mathrm{E}-04$ & $-1.17 \mathrm{E}-01$ & $-1.04 \mathrm{E}+00$ & 4.05E-04 & $2.99 \mathrm{E}-04$ & $-1.73 \mathrm{E}-03$ \\
\hline $\mathrm{x}_{10}$ & $*$ & $\mathrm{x}_{12}$ & $-1.41 \mathrm{E}-01$ & $-5.52 \mathrm{E}-01$ & $-2.90 \mathrm{E}-05$ & $-6.50 \mathrm{E}-01$ & 1.62E-01 & 4.19E-03 & $2.56 \mathrm{E}-04$ & $-2.19 \mathrm{E}-03$ \\
\hline $\mathrm{x}_{10}$ & $*$ & $\mathrm{x}_{13}$ & $7.18 \mathrm{E}-04$ & $5.31 \mathrm{E}-02$ & $-1.24 \mathrm{E}-04$ & 3.79E-02 & $-3.24 \mathrm{E}-01$ & $1.74 \mathrm{E}-04$ & $-3.74 \mathrm{E}-05$ & $2.38 \mathrm{E}-04$ \\
\hline $\mathrm{x}_{10}$ & $*$ & $\mathrm{x}_{14}$ & $1.03 \mathrm{E}-02$ & $9.05 \mathrm{E}-02$ & $-1.58 \mathrm{E}-04$ & 7.33E-02 & $-9.60 \mathrm{E}-01$ & $5.99 \mathrm{E}-04$ & $-3.60 \mathrm{E}-05$ & $-2.44 \mathrm{E}-04$ \\
\hline $\mathrm{x}_{11}$ & $*$ & $\mathrm{x}_{12}$ & $6.21 \mathrm{E}-02$ & $4.60 \mathrm{E}+00$ & $2.15 \mathrm{E}-02$ & $3.57 \mathrm{E}+00$ & $-2.66 \mathrm{E}+01$ & $-8.62 \mathrm{E}-03$ & $-2.62 \mathrm{E}-03$ & $7.09 \mathrm{E}-03$ \\
\hline $\mathrm{x}_{11}$ & $*$ & $\mathrm{x}_{13}$ & $8.35 \mathrm{E}-02$ & $6.27 \mathrm{E}-01$ & $-5.51 \mathrm{E}-04$ & $6.51 \mathrm{E}-01$ & $-4.80 \mathrm{E}+00$ & $-1.07 \mathrm{E}-03$ & $2.85 \mathrm{E}-04$ & $1.88 \mathrm{E}-04$ \\
\hline $\mathrm{x}_{11}$ & $*$ & $\mathrm{x}_{14}$ & $-5.65 \mathrm{E}-02$ & $2.29 \mathrm{E}+00$ & $1.36 \mathrm{E}-02$ & $2.67 \mathrm{E}+00$ & $-3.08 \mathrm{E}+01$ & $-1.31 \mathrm{E}-02$ & $3.53 \mathrm{E}-03$ & $-6.89 \mathrm{E}-03$ \\
\hline $\mathrm{x}_{12}$ & $*$ & $\mathrm{x}_{13}$ & $-5.49 \mathrm{E}-01$ & $-4.43 \mathrm{E}+00$ & $-2.42 \mathrm{E}-02$ & $-7.08 \mathrm{E}+00$ & $4.28 \mathrm{E}+01$ & $5.70 \mathrm{E}-02$ & $-7.31 \mathrm{E}-03$ & $-4.98 \mathrm{E}-03$ \\
\hline $\mathrm{x}_{12}$ & $*$ & $\mathrm{x}_{14}$ & $-4.07 \mathrm{E}-01$ & $-2.96 \mathrm{E}+00$ & $3.42 \mathrm{E}-03$ & $-1.25 \mathrm{E}+00$ & $1.75 \mathrm{E}+01$ & $-1.69 \mathrm{E}-02$ & $-1.54 \mathrm{E}-03$ & $-1.32 \mathrm{E}-02$ \\
\hline \multirow[t]{3}{*}{$\mathrm{x}_{13}$} & $*$ & $\mathrm{x}_{14}$ & $-1.33 \mathrm{E}-01$ & $-1.22 \mathrm{E}+00$ & $-1.05 \mathrm{E}-02$ & $-2.32 \mathrm{E}+00$ & $1.42 \mathrm{E}+01$ & $2.22 \mathrm{E}-02$ & $-3.01 \mathrm{E}-03$ & $-8.78 \mathrm{E}-04$ \\
\hline & $\mathrm{c}$ & & $-4.00 \mathrm{E}-03$ & $-4.16 \mathrm{E}-02$ & $2.12 \mathrm{E}-04$ & $-4.86 \mathrm{E}-02$ & $2.63 \mathrm{E}-01$ & $6.20 \mathrm{E}-05$ & 2.17E-05 & $-1.46 \mathrm{E}-04$ \\
\hline & $r^{2}$ & & 1 & 1 & 1 & 1 & 1 & 1 & 1 & 1 \\
\hline
\end{tabular}

Table 7. The $\mathrm{x}$ and $\mathrm{y}$ variables, their coefficient and determination coefficient of equations belonging non-linear models yielded from the data collected Station 4

\begin{tabular}{rrrrrrrrc}
\hline & $\mathrm{y}_{1}$ & \multicolumn{1}{c}{$\mathrm{y}_{2}$} & \multicolumn{1}{c}{$\mathrm{y}_{3}$} & \multicolumn{1}{c}{$\mathrm{y}_{4}$} & $\mathrm{y}_{5}$ & $\mathrm{y}_{6}$ & $\mathrm{y}_{7}$ & $\mathrm{y}_{8}$ \\
\hline $\mathrm{x}_{1}$ & $-2.26 \mathrm{E}-01$ & $-1.64 \mathrm{E}+00$ & $2.24 \mathrm{E}-03$ & $-1.17 \mathrm{E}+00$ & $3.24 \mathrm{E}+00$ & $-2.14 \mathrm{E}-03$ & $1.83 \mathrm{E}-03$ & $-2.47 \mathrm{E}-02$ \\
$\mathrm{x}_{2}$ & $4.55 \mathrm{E}-03$ & $1.26 \mathrm{E}-01$ & $-6.58 \mathrm{E}-04$ & $-1.84 \mathrm{E}-02$ & $3.07 \mathrm{E}+00$ & $8.01 \mathrm{E}-05$ & $-9.87 \mathrm{E}-05$ & $3.25 \mathrm{E}-03$ \\
$\mathrm{x}_{3}$ & $-1.04 \mathrm{E}-01$ & $-3.16 \mathrm{E}-01$ & $2.48 \mathrm{E}-03$ & $-5.24 \mathrm{E}-01$ & $-2.94 \mathrm{E}+00$ & $-5.87 \mathrm{E}-04$ & $1.06 \mathrm{E}-03$ & $-9.22 \mathrm{E}-03$ \\
$\mathrm{x}_{4}$ & $1.86 \mathrm{E}-02$ & $9.83 \mathrm{E}-01$ & $2.89 \mathrm{E}-03$ & $-1.02 \mathrm{E}-01$ & $3.38 \mathrm{E}+00$ & $4.07 \mathrm{E}-03$ & $-1.18 \mathrm{E}-03$ & $-2.83 \mathrm{E}-03$
\end{tabular}




\begin{tabular}{|c|c|c|c|c|c|c|c|c|c|c|}
\hline \multicolumn{3}{|c|}{$\mathrm{x}_{5}$} & $-2.05 \mathrm{E}-01$ & $-2.42 \mathrm{E}+00$ & $5.62 \mathrm{E}-03$ & $-1.46 \mathrm{E}+00$ & $7.97 \mathrm{E}+01$ & $-7.82 \mathrm{E}-04$ & $1.45 \mathrm{E}-03$ & $3.88 \mathrm{E}-02$ \\
\hline & $\mathrm{x}_{6}$ & & $1.99 \mathrm{E}-02$ & $-2.82 \mathrm{E}-01$ & $-6.67 \mathrm{E}-04$ & $-2.72 \mathrm{E}-02$ & $9.95 \mathrm{E}+00$ & $1.07 \mathrm{E}-03$ & $-7.46 \mathrm{E}-04$ & $5.47 \mathrm{E}-03$ \\
\hline & $\mathrm{x}_{7}$ & & $-1.18 \mathrm{E}-01$ & $-2.36 \mathrm{E}+00$ & $-3.58 \mathrm{E}-03$ & $-9.82 \mathrm{E}-01$ & $8.18 \mathrm{E}+01$ & $-2.67 \mathrm{E}-03$ & 4.24E-04 & $3.82 \mathrm{E}-02$ \\
\hline & $\mathrm{x}_{8}$ & & $1.07 \mathrm{E}-02$ & $2.51 \mathrm{E}+00$ & $5.00 \mathrm{E}-03$ & $8.40 \mathrm{E}-01$ & $-1.78 \mathrm{E}+01$ & $8.31 \mathrm{E}-04$ & $5.88 \mathrm{E}-05$ & $1.29 \mathrm{E}-02$ \\
\hline & $\mathrm{x}_{9}$ & & $-2.75 \mathrm{E}-03$ & $5.98 \mathrm{E}-02$ & $-2.97 \mathrm{E}-05$ & $9.39 \mathrm{E}-05$ & 4.09E-01 & $-2.37 \mathrm{E}-05$ & $1.53 \mathrm{E}-05$ & $6.75 \mathrm{E}-04$ \\
\hline & $\mathrm{x}_{10}$ & & 4.06E-04 & $3.36 \mathrm{E}-02$ & $5.62 \mathrm{E}-05$ & $5.70 \mathrm{E}-03$ & $-2.31 \mathrm{E}-01$ & $2.68 \mathrm{E}-05$ & $5.10 \mathrm{E}-06$ & $1.09 \mathrm{E}-04$ \\
\hline & $\mathrm{x}_{11}$ & & $-6.74 \mathrm{E}-03$ & 7.57E-02 & $1.20 \mathrm{E}-03$ & $1.69 \mathrm{E}-01$ & $-7.20 \mathrm{E}+00$ & $-5.90 \mathrm{E}-04$ & $3.48 \mathrm{E}-04$ & $-5.57 \mathrm{E}-04$ \\
\hline & $\mathrm{x}_{12}$ & & $2.66 \mathrm{E}-02$ & $-7.23 \mathrm{E}-01$ & $3.70 \mathrm{E}-04$ & $-5.97 \mathrm{E}-01$ & $3.47 \mathrm{E}+00$ & $1.97 \mathrm{E}-03$ & $2.56 \mathrm{E}-04$ & $-1.59 \mathrm{E}-02$ \\
\hline & $\mathrm{x}_{13}$ & & $-1.87 \mathrm{E}-03$ & $-1.37 \mathrm{E}-01$ & $-1.22 \mathrm{E}-04$ & $-7.55 \mathrm{E}-03$ & $1.58 \mathrm{E}+00$ & $-3.36 \mathrm{E}-05$ & $-8.59 \mathrm{E}-05$ & $1.05 \mathrm{E}-03$ \\
\hline & $\mathrm{x}_{14}$ & & $-3.12 \mathrm{E}-02$ & $-1.39 \mathrm{E}+00$ & $1.41 \mathrm{E}-03$ & $-3.66 \mathrm{E}-01$ & $1.20 \mathrm{E}+00$ & $3.30 \mathrm{E}-04$ & $2.18 \mathrm{E}-04$ & $-1.40 \mathrm{E}-02$ \\
\hline $\mathrm{x}_{1}$ & $*$ & $\mathrm{x}_{1}$ & $3.28 \mathrm{E}-02$ & $-1.48 \mathrm{E}+00$ & $4.86 \mathrm{E}-03$ & $-1.55 \mathrm{E}-01$ & $-1.56 \mathrm{E}+00$ & 3.64E-03 & $-1.69 \mathrm{E}-03$ & $-9.62 \mathrm{E}-03$ \\
\hline $\mathrm{x}_{2}$ & $*$ & $\mathrm{x}_{2}$ & $3.27 \mathrm{E}-02$ & $1.17 \mathrm{E}+00$ & $-3.83 \mathrm{E}-03$ & $-9.94 \mathrm{E}-03$ & $1.45 \mathrm{E}+01$ & $6.95 \mathrm{E}-05$ & $-2.98 \mathrm{E}-04$ & $2.19 \mathrm{E}-02$ \\
\hline $\mathrm{x}_{3}$ & $*$ & $\mathrm{x}_{3}$ & 3.92E-01 & $2.73 \mathrm{E}+00$ & $2.96 \mathrm{E}-02$ & $5.66 \mathrm{E}+00$ & $-1.16 \mathrm{E}+02$ & 8.14E-03 & $-3.70 \mathrm{E}-03$ & $-5.90 \mathrm{E}-02$ \\
\hline $\mathrm{x}_{4}$ & $*$ & $\mathrm{x}_{4}$ & $-6.44 \mathrm{E}-02$ & $-1.25 \mathrm{E}+00$ & $-2.98 \mathrm{E}-03$ & $-1.58 \mathrm{E}-01$ & $9.74 \mathrm{E}+00$ & $-1.48 \mathrm{E}-03$ & $-2.95 \mathrm{E}-04$ & $-1.52 \mathrm{E}-03$ \\
\hline $\mathrm{x}_{5}$ & $*$ & $\mathrm{x}_{5}$ & $1.85 \mathrm{E}-04$ & $1.18 \mathrm{E}-02$ & $-5.79 \mathrm{E}-05$ & $5.34 \mathrm{E}-03$ & $2.15 \mathrm{E}-02$ & $-5.87 \mathrm{E}-05$ & $3.96 \mathrm{E}-06$ & $1.70 \mathrm{E}-04$ \\
\hline $\mathrm{x}_{6}$ & $*$ & $\mathrm{x}_{6}$ & $1.93 \mathrm{E}-01$ & $-2.49 \mathrm{E}+00$ & $-2.42 \mathrm{E}-03$ & $1.45 \mathrm{E}-01$ & $5.36 \mathrm{E}+01$ & $9.48 \mathrm{E}-03$ & $-6.30 \mathrm{E}-03$ & $2.52 \mathrm{E}-02$ \\
\hline$x_{7}$ & $*$ & $\mathrm{x}_{7}$ & $-3.13 \mathrm{E}-03$ & $6.42 \mathrm{E}-03$ & $4.31 \mathrm{E}-05$ & $-1.92 \mathrm{E}-02$ & $-2.04 \mathrm{E}-01$ & $6.30 \mathrm{E}-05$ & $-1.64 \mathrm{E}-05$ & $-5.36 \mathrm{E}-04$ \\
\hline $\mathrm{x}_{8}$ & $*$ & $\mathrm{x}_{8}$ & $6.85 \mathrm{E}-05$ & 4.53E-04 & $4.58 \mathrm{E}-06$ & 4.46E-04 & $-1.51 \mathrm{E}-02$ & 5.31E-06 & $-2.69 \mathrm{E}-06$ & $-7.20 \mathrm{E}-07$ \\
\hline $\mathrm{X}_{9}$ & $*$ & $\mathrm{x}_{9}$ & $-4.17 \mathrm{E}-02$ & $-1.17 \mathrm{E}-01$ & $-9.91 \mathrm{E}-04$ & $-5.59 \mathrm{E}-02$ & $2.91 \mathrm{E}+00$ & $-1.19 \mathrm{E}-03$ & $2.46 \mathrm{E}-04$ & 2.74E-04 \\
\hline $\mathrm{x}_{10}$ & $*$ & $\mathrm{x}_{10}$ & $2.99 \mathrm{E}-12$ & $-9.47 \mathrm{E}-11$ & $3.75 \mathrm{E}-13$ & 3.96E-11 & $-1.53 \mathrm{E}-09$ & 4.94E-14 & $-1.55 \mathrm{E}-14$ & $-1.64 \mathrm{E}-12$ \\
\hline $\mathrm{x}_{11}$ & $*$ & $\mathrm{x}_{11}$ & 4.17E-01 & $5.03 \mathrm{E}+00$ & $-2.00 \mathrm{E}-03$ & $9.66 \mathrm{E}-01$ & $1.45 \mathrm{E}+01$ & $1.11 \mathrm{E}-02$ & $-4.52 \mathrm{E}-03$ & 4.90E-02 \\
\hline $\mathrm{x}_{12}$ & $*$ & $\mathrm{x}_{12}$ & $2.20 \mathrm{E}-01$ & 7.09E-01 & $1.81 \mathrm{E}-02$ & $2.23 \mathrm{E}+00$ & $-6.85 \mathrm{E}+01$ & 7.30E-03 & $-1.32 \mathrm{E}-03$ & $-8.78 \mathrm{E}-03$ \\
\hline$x_{13}$ & $*$ & $\mathrm{x}_{13}$ & $-6.24 \mathrm{E}-02$ & $-1.30 \mathrm{E}+00$ & $9.12 \mathrm{E}-04$ & $-2.64 \mathrm{E}-01$ & $-8.94 \mathrm{E}-01$ & $-8.85 \mathrm{E}-04$ & $3.40 \mathrm{E}-04$ & $-1.54 \mathrm{E}-02$ \\
\hline $\mathrm{x}_{14}$ & $*$ & $\mathrm{x}_{14}$ & 4.73E-01 & $1.89 \mathrm{E}-03$ & $-7.22 \mathrm{E}-03$ & $3.49 \mathrm{E}+00$ & $-6.30 \mathrm{E}+00$ & $5.78 \mathrm{E}-05$ & $-5.05 \mathrm{E}-03$ & $2.35 \mathrm{E}-02$ \\
\hline $\mathrm{x}_{1}$ & $*$ & $\mathrm{x}_{2}$ & $3.30 \mathrm{E}-03$ & $1.43 \mathrm{E}+00$ & $-2.95 \mathrm{E}-03$ & $-1.76 \mathrm{E}-01$ & $2.48 \mathrm{E}+01$ & $6.34 \mathrm{E}-03$ & $-3.90 \mathrm{E}-03$ & $3.47 \mathrm{E}-02$ \\
\hline $\mathrm{x}_{1}$ & $*$ & $\mathrm{x}_{3}$ & $-5.35 \mathrm{E}-01$ & $6.95 \mathrm{E}-01$ & $-6.75 \mathrm{E}-03$ & $-2.69 \mathrm{E}+00$ & $5.47 \mathrm{E}+00$ & $-9.67 \mathrm{E}-03$ & 7.47E-03 & $-1.00 \mathrm{E}-02$ \\
\hline $\mathrm{x}_{1}$ & $*$ & $\mathrm{x}_{4}$ & $4.57 \mathrm{E}-02$ & $1.39 \mathrm{E}+00$ & $-1.31 \mathrm{E}-02$ & $3.50 \mathrm{E}-01$ & $2.93 \mathrm{E}+01$ & $-9.63 \mathrm{E}-04$ & $-7.58 \mathrm{E}-04$ & $3.94 \mathrm{E}-02$ \\
\hline $\mathrm{x}_{1}$ & $*$ & $\mathrm{x}_{5}$ & $-2.35 \mathrm{E}-02$ & $-4.03 \mathrm{E}-02$ & $-1.59 \mathrm{E}-04$ & $-8.83 \mathrm{E}-02$ & $-1.26 \mathrm{E}+00$ & $-9.71 \mathrm{E}-04$ & 4.41E-05 & $-1.76 \mathrm{E}-03$ \\
\hline $\mathrm{x}_{1}$ & $*$ & $\mathrm{x}_{6}$ & $-7.81 \mathrm{E}-01$ & $-4.59 \mathrm{E}+00$ & $-9.75 \mathrm{E}-03$ & $-4.01 \mathrm{E}+00$ & $8.13 \mathrm{E}+01$ & $-1.01 \mathrm{E}-02$ & $3.98 \mathrm{E}-03$ & $-1.66 \mathrm{E}-02$ \\
\hline $\mathrm{x}_{1}$ & $*$ & $\mathrm{x}_{7}$ & $5.25 \mathrm{E}-02$ & $1.41 \mathrm{E}-01$ & $6.20 \mathrm{E}-04$ & $2.75 \mathrm{E}-01$ & $-9.89 \mathrm{E}-01$ & $1.19 \mathrm{E}-03$ & $-1.64 \mathrm{E}-04$ & $1.38 \mathrm{E}-03$ \\
\hline $\mathrm{x}_{1}$ & $*$ & $\mathrm{x}_{8}$ & $3.12 \mathrm{E}-02$ & $1.73 \mathrm{E}-01$ & $2.46 \mathrm{E}-03$ & $3.58 \mathrm{E}-01$ & $-1.07 \mathrm{E}+01$ & $-1.35 \mathrm{E}-04$ & $8.51 \mathrm{E}-04$ & $-4.09 \mathrm{E}-03$ \\
\hline $\mathrm{x}_{1}$ & $*$ & $\mathrm{x}_{9}$ & $2.46 \mathrm{E}-01$ & $-1.82 \mathrm{E}-01$ & $1.21 \mathrm{E}-02$ & $1.65 \mathrm{E}+00$ & $-5.55 \mathrm{E}+01$ & $1.86 \mathrm{E}-03$ & 8.44E-04 & $-1.90 \mathrm{E}-02$ \\
\hline $\mathrm{x}_{1}$ & $*$ & $\mathrm{x}_{10}$ & $5.34 \mathrm{E}-02$ & 7.40E-01 & $-1.55 \mathrm{E}-03$ & $1.34 \mathrm{E}-01$ & $3.80 \mathrm{E}+00$ & $2.36 \mathrm{E}-04$ & $-2.37 \mathrm{E}-04$ & $1.21 \mathrm{E}-02$ \\
\hline $\mathrm{x}_{1}$ & * & $\mathrm{x}_{11}$ & 4.07E-01 & $6.71 \mathrm{E}+00$ & $1.06 \mathrm{E}-02$ & $1.15 \mathrm{E}+00$ & $-2.13 \mathrm{E}+01$ & $1.59 \mathrm{E}-02$ & $-1.27 \mathrm{E}-03$ & 7.15E-02 \\
\hline $\mathrm{x}_{1}$ & $*$ & $\mathrm{x}_{12}$ & 3.33E-01 & $3.93 \mathrm{E}+00$ & $-5.08 \mathrm{E}-03$ & $2.02 \mathrm{E}+00$ & $1.38 \mathrm{E}+01$ & 7.33E-03 & $-3.68 \mathrm{E}-03$ & $3.95 \mathrm{E}-02$ \\
\hline $\mathrm{x}_{1}$ & $*$ & $\mathrm{x}_{13}$ & $3.22 \mathrm{E}-01$ & $5.30 \mathrm{E}+00$ & $3.00 \mathrm{E}-02$ & $2.67 \mathrm{E}+00$ & $-1.10 \mathrm{E}+02$ & $1.42 \mathrm{E}-02$ & $-2.42 \mathrm{E}-03$ & $-2.59 \mathrm{E}-02$ \\
\hline $\mathrm{x}_{1}$ & $*$ & $\mathrm{x}_{14}$ & $1.29 \mathrm{E}-01$ & $-2.80 \mathrm{E}+00$ & $-5.84 \mathrm{E}-05$ & $-8.04 \mathrm{E}-01$ & $-9.04 \mathrm{E}+00$ & $1.36 \mathrm{E}-04$ & $9.10 \mathrm{E}-04$ & $-8.68 \mathrm{E}-03$ \\
\hline $\mathrm{x}_{2}$ & $*$ & $\mathrm{x}_{3}$ & $1.69 \mathrm{E}-01$ & $3.04 \mathrm{E}+00$ & $-2.88 \mathrm{E}-03$ & $7.09 \mathrm{E}-01$ & $1.58 \mathrm{E}+01$ & $6.19 \mathrm{E}-03$ & $-3.58 \mathrm{E}-03$ & 4.57E-02 \\
\hline $\mathrm{x}_{2}$ & $*$ & $\mathrm{x}_{4}$ & $-4.44 \mathrm{E}-02$ & $2.95 \mathrm{E}+00$ & $9.75 \mathrm{E}-03$ & $4.55 \mathrm{E}-01$ & $-3.98 \mathrm{E}+01$ & $5.88 \mathrm{E}-04$ & $1.48 \mathrm{E}-03$ & $-1.39 \mathrm{E}-02$ \\
\hline $\mathrm{x}_{2}$ & $*$ & $\mathrm{x}_{5}$ & $1.01 \mathrm{E}-01$ & $-2.67 \mathrm{E}+00$ & $-1.23 \mathrm{E}-02$ & $-1.77 \mathrm{E}+00$ & $5.92 \mathrm{E}+01$ & $-1.48 \mathrm{E}-03$ & $-2.60 \mathrm{E}-04$ & $1.75 \mathrm{E}-02$ \\
\hline $\mathrm{x}_{2}$ & $*$ & $\mathrm{x}_{6}$ & $5.35 \mathrm{E}-02$ & $1.73 \mathrm{E}+00$ & $-4.45 \mathrm{E}-03$ & $-6.18 \mathrm{E}-02$ & $2.03 \mathrm{E}+01$ & $1.60 \mathrm{E}-03$ & $-9.52 \mathrm{E}-04$ & $2.80 \mathrm{E}-02$ \\
\hline $\mathrm{x}_{2}$ & $*$ & $\mathrm{x}_{7}$ & $-2.23 \mathrm{E}-01$ & $1.11 \mathrm{E}+00$ & $2.12 \mathrm{E}-02$ & $2.23 \mathrm{E}+00$ & $-9.92 \mathrm{E}+01$ & $1.75 \mathrm{E}-03$ & $1.82 \mathrm{E}-03$ & $-6.01 \mathrm{E}-02$ \\
\hline $\mathrm{x}_{2}$ & $*$ & $\mathrm{x}_{8}$ & $2.28 \mathrm{E}-01$ & $6.93 \mathrm{E}+00$ & $-1.78 \mathrm{E}-02$ & 7.87E-01 & $7.82 \mathrm{E}+01$ & $2.30 \mathrm{E}-03$ & $-1.56 \mathrm{E}-03$ & $1.01 \mathrm{E}-01$ \\
\hline $\mathrm{X}_{2}$ & * & $\mathrm{x}_{9}$ & $1.94 \mathrm{E}-02$ & $8.54 \mathrm{E}-01$ & $-7.37 \mathrm{E}-04$ & $1.12 \mathrm{E}-01$ & $2.31 \mathrm{E}+00$ & $2.49 \mathrm{E}-04$ & $-5.13 \mathrm{E}-05$ & $1.08 \mathrm{E}-02$ \\
\hline $\mathrm{x}_{2}$ & $*$ & $\mathrm{x}_{10}$ & $-6.14 \mathrm{E}-03$ & $4.12 \mathrm{E}-01$ & $-6.97 \mathrm{E}-05$ & $-1.22 \mathrm{E}-01$ & $8.71 \mathrm{E}-01$ & $3.17 \mathrm{E}-04$ & $6.42 \mathrm{E}-05$ & $1.22 \mathrm{E}-03$ \\
\hline
\end{tabular}


quality

\begin{tabular}{|c|c|c|c|c|c|c|c|c|c|c|}
\hline $\mathrm{x}_{2}$ & $*$ & $\mathrm{x}_{11}$ & $6.38 \mathrm{E}-02$ & 2.12E-01 & $-5.85 \mathrm{E}-03$ & $1.29 \mathrm{E}-01$ & $1.88 \mathrm{E}+01$ & $-1.06 \mathrm{E}-03$ & $-6.19 \mathrm{E}-04$ & $2.33 \mathrm{E}-02$ \\
\hline $\mathrm{x}_{2}$ & $*$ & $\mathrm{x}_{12}$ & $1.22 \mathrm{E}-01$ & $8.19 \mathrm{E}+00$ & $-2.17 \mathrm{E}-02$ & $-5.27 \mathrm{E}-01$ & $7.78 \mathrm{E}+01$ & $3.21 \mathrm{E}-03$ & $-3.04 \mathrm{E}-03$ & $1.39 \mathrm{E}-01$ \\
\hline $\mathrm{x}_{2}$ & $*$ & $\mathrm{x}_{13}$ & $3.13 \mathrm{E}-02$ & 2.94E-01 & $-1.88 \mathrm{E}-03$ & $5.24 \mathrm{E}-02$ & $8.07 \mathrm{E}+00$ & $1.87 \mathrm{E}-04$ & $-3.14 \mathrm{E}-04$ & $1.03 \mathrm{E}-02$ \\
\hline $\mathrm{x}_{2}$ & $*$ & $\mathrm{x}_{14}$ & 1.42E-01 & $3.37 \mathrm{E}+00$ & $-8.85 \mathrm{E}-03$ & $3.41 \mathrm{E}-01$ & $3.36 \mathrm{E}+01$ & $4.25 \mathrm{E}-03$ & $-3.47 \mathrm{E}-03$ & $6.33 \mathrm{E}-02$ \\
\hline $\mathrm{x}_{3}$ & $*$ & $\mathrm{x}_{4}$ & $-1.96 \mathrm{E}-01$ & 4.09E-01 & $2.95 \mathrm{E}-02$ & $2.24 \mathrm{E}+00$ & $-1.12 \mathrm{E}+02$ & $-4.28 \mathrm{E}-03$ & $2.83 \mathrm{E}-03$ & $-8.76 \mathrm{E}-02$ \\
\hline $\mathrm{x}_{3}$ & $*$ & $\mathrm{x}_{5}$ & $3.22 \mathrm{E}-02$ & $-4.27 \mathrm{E}-01$ & $1.82 \mathrm{E}-03$ & $5.75 \mathrm{E}-02$ & $-1.84 \mathrm{E}+00$ & 3.91E-04 & $2.43 \mathrm{E}-04$ & $-1.45 \mathrm{E}-03$ \\
\hline $\mathrm{x}_{3}$ & $*$ & $\mathrm{x}_{6}$ & $-2.42 \mathrm{E}-01$ & 2.39E-01 & $4.81 \mathrm{E}-03$ & $-1.23 \mathrm{E}+00$ & $-5.25 \mathrm{E}+00$ & $-1.18 \mathrm{E}-03$ & $2.31 \mathrm{E}-03$ & $2.54 \mathrm{E}-03$ \\
\hline $\mathrm{x}_{3}$ & * & $\mathrm{x}_{7}$ & $-3.89 \mathrm{E}-02$ & $5.15 \mathrm{E}-01$ & $-5.21 \mathrm{E}-03$ & $-3.35 \mathrm{E}-01$ & $1.20 \mathrm{E}+01$ & $-6.49 \mathrm{E}-05$ & $-5.14 \mathrm{E}-04$ & $8.06 \mathrm{E}-03$ \\
\hline $\mathrm{x}_{3}$ & $*$ & $\mathrm{x}_{8}$ & $-7.65 \mathrm{E}-03$ & $1.68 \mathrm{E}-01$ & $-1.34 \mathrm{E}-03$ & $-1.20 \mathrm{E}-01$ & $3.35 \mathrm{E}+00$ & $2.43 \mathrm{E}-05$ & $-5.54 \mathrm{E}-04$ & $1.67 \mathrm{E}-03$ \\
\hline $\mathrm{x}_{3}$ & * & $\mathrm{X}_{9}$ & $1.44 \mathrm{E}-01$ & $6.01 \mathrm{E}-01$ & 1.19E-02 & $1.25 \mathrm{E}+00$ & $-5.30 \mathrm{E}+01$ & $1.70 \mathrm{E}-03$ & $1.03 \mathrm{E}-03$ & $-1.88 \mathrm{E}-02$ \\
\hline $\mathrm{x}_{3}$ & * & $\mathrm{x}_{10}$ & 8.79E-03 & $1.53 \mathrm{E}-01$ & $-4.23 \mathrm{E}-03$ & $-1.71 \mathrm{E}-01$ & $1.47 \mathrm{E}+01$ & $-1.21 \mathrm{E}-03$ & $-4.94 \mathrm{E}-05$ & $1.44 \mathrm{E}-02$ \\
\hline $\mathrm{x}_{3}$ & * & $\mathrm{x}_{11}$ & $3.98 \mathrm{E}-01$ & $-4.55 \mathrm{E}+00$ & $2.74 \mathrm{E}-04$ & $1.43 \mathrm{E}+00$ & $-3.94 \mathrm{E}+00$ & $4.88 \mathrm{E}-03$ & $-3.10 \mathrm{E}-03$ & $-1.33 \mathrm{E}-02$ \\
\hline $\mathrm{x}_{3}$ & * & $\mathrm{x}_{12}$ & $-1.70 \mathrm{E}-01$ & $-5.82 \mathrm{E}+00$ & $-5.70 \mathrm{E}-03$ & $-1.32 \mathrm{E}+00$ & $2.87 \mathrm{E}+01$ & $-1.15 \mathrm{E}-02$ & $1.85 \mathrm{E}-03$ & $-5.61 \mathrm{E}-03$ \\
\hline $\mathrm{x}_{3}$ & * & $\mathrm{x}_{13}$ & $3.49 \mathrm{E}-01$ & $6.87 \mathrm{E}+00$ & $1.89 \mathrm{E}-02$ & $2.29 \mathrm{E}+00$ & $-6.91 \mathrm{E}+01$ & $1.22 \mathrm{E}-02$ & $-2.51 \mathrm{E}-03$ & $2.54 \mathrm{E}-02$ \\
\hline $\mathrm{x}_{3}$ & $*$ & $\mathrm{x}_{14}$ & 8.03E-02 & $3.92 \mathrm{E}+00$ & $-1.71 \mathrm{E}-02$ & $-2.28 \mathrm{E}+00$ & $8.23 \mathrm{E}+01$ & $3.19 \mathrm{E}-03$ & $-2.85 \mathrm{E}-03$ & $8.59 \mathrm{E}-02$ \\
\hline $\mathrm{x}_{4}$ & $*$ & $\mathrm{X}_{5}$ & $1.90 \mathrm{E}-02$ & $-1.95 \mathrm{E}-01$ & $-5.47 \mathrm{E}-04$ & $-3.94 \mathrm{E}-02$ & $1.42 \mathrm{E}+00$ & 4.14E-05 & $-5.89 \mathrm{E}-05$ & $-9.54 \mathrm{E}-04$ \\
\hline $\mathrm{x}_{4}$ & $*$ & $\mathrm{x}_{6}$ & $1.46 \mathrm{E}-01$ & $9.87 \mathrm{E}-01$ & $3.14 \mathrm{E}-03$ & $-8.39 \mathrm{E}-01$ & $1.02 \mathrm{E}+02$ & $2.59 \mathrm{E}-02$ & $-1.16 \mathrm{E}-02$ & $-1.05 \mathrm{E}-02$ \\
\hline $\mathrm{x}_{4}$ & * & $\mathrm{x}_{7}$ & $-2.44 \mathrm{E}-02$ & $3.48 \mathrm{E}-01$ & $1.12 \mathrm{E}-03$ & $6.55 \mathrm{E}-02$ & $-5.69 \mathrm{E}+00$ & $-5.50 \mathrm{E}-04$ & 4.95E-04 & $1.51 \mathrm{E}-03$ \\
\hline $\mathrm{x}_{4}$ & $*$ & $\mathrm{x}_{8}$ & $-7.65 \mathrm{E}-03$ & $1.68 \mathrm{E}-01$ & $-1.34 \mathrm{E}-03$ & $-1.20 \mathrm{E}-01$ & $3.35 \mathrm{E}+00$ & $2.43 \mathrm{E}-05$ & $-5.54 \mathrm{E}-04$ & $1.67 \mathrm{E}-03$ \\
\hline $\mathrm{x}_{4}$ & $*$ & $\mathrm{x}_{9}$ & $-2.17 \mathrm{E}-01$ & $2.66 \mathrm{E}+00$ & $-5.90 \mathrm{E}-03$ & $-5.35 \mathrm{E}-01$ & $2.28 \mathrm{E}+01$ & $-4.27 \mathrm{E}-03$ & $1.70 \mathrm{E}-03$ & $3.01 \mathrm{E}-02$ \\
\hline $\mathrm{x}_{4}$ & $*$ & $\mathrm{x}_{10}$ & $5.05 \mathrm{E}-02$ & 6.61E-01 & $-4.12 \mathrm{E}-03$ & $1.71 \mathrm{E}-01$ & $1.55 \mathrm{E}+01$ & $-3.47 \mathrm{E}-04$ & $-4.01 \mathrm{E}-04$ & $2.25 \mathrm{E}-02$ \\
\hline $\mathrm{x}_{4}$ & $*$ & $\mathrm{x}_{11}$ & $3.82 \mathrm{E}-02$ & $-4.39 \mathrm{E}+00$ & $-5.98 \mathrm{E}-02$ & 9.47E-01 & $1.96 \mathrm{E}+02$ & $-2.25 \mathrm{E}-02$ & $-6.60 \mathrm{E}-04$ & $2.38 \mathrm{E}-01$ \\
\hline $\mathrm{x}_{4}$ & $*$ & $\mathrm{x}_{12}$ & $5.52 \mathrm{E}-02$ & 4.27E-01 & $-9.99 \mathrm{E}-04$ & 7.40E-01 & $-2.32 \mathrm{E}+01$ & $-6.47 \mathrm{E}-03$ & $3.83 \mathrm{E}-03$ & $5.51 \mathrm{E}-03$ \\
\hline $\mathrm{x}_{4}$ & $*$ & $\mathrm{x}_{13}$ & 4.15E-02 & $-7.72 \mathrm{E}+00$ & $2.31 \mathrm{E}-02$ & $9.56 \mathrm{E}-01$ & $-8.99 \mathrm{E}+01$ & $3.32 \mathrm{E}-03$ & $-1.06 \mathrm{E}-03$ & $-1.22 \mathrm{E}-01$ \\
\hline $\mathrm{x}_{4}$ & $*$ & $\mathrm{x}_{14}$ & $1.30 \mathrm{E}-02$ & $-1.49 \mathrm{E}+00$ & $-3.15 \mathrm{E}-03$ & $-2.84 \mathrm{E}+00$ & $6.52 \mathrm{E}+01$ & $1.35 \mathrm{E}-02$ & $-6.64 \mathrm{E}-03$ & $1.19 \mathrm{E}-02$ \\
\hline $\mathrm{x}_{5}$ & * & $\mathrm{x}_{6}$ & $-1.02 \mathrm{E}-01$ & $9.20 \mathrm{E}-01$ & $9.10 \mathrm{E}-03$ & $-2.85 \mathrm{E}-01$ & $-3.42 \mathrm{E}+01$ & $5.08 \mathrm{E}-03$ & $-7.81 \mathrm{E}-04$ & $-2.83 \mathrm{E}-02$ \\
\hline $\mathrm{x}_{5}$ & $*$ & $\mathrm{x}_{7}$ & $2.18 \mathrm{E}-03$ & $-2.68 \mathrm{E}-02$ & $1.19 \mathrm{E}-06$ & $-1.37 \mathrm{E}-03$ & $2.04 \mathrm{E}-01$ & $1.02 \mathrm{E}-04$ & $-7.54 \mathrm{E}-06$ & $-9.35 \mathrm{E}-07$ \\
\hline $\mathrm{x}_{5}$ & * & $\mathrm{x}_{8}$ & $-5.55 \mathrm{E}-03$ & $-1.85 \mathrm{E}-02$ & $1.77 \mathrm{E}-06$ & $2.79 \mathrm{E}-02$ & $3.98 \mathrm{E}-01$ & $-7.59 \mathrm{E}-05$ & $1.98 \mathrm{E}-05$ & $1.14 \mathrm{E}-03$ \\
\hline $\mathrm{x}_{5}$ & $*$ & $\mathrm{x}_{9}$ & $-4.21 \mathrm{E}-01$ & $-2.57 \mathrm{E}+00$ & $-5.66 \mathrm{E}-03$ & $-1.81 \mathrm{E}+00$ & $1.41 \mathrm{E}+01$ & $-6.73 \mathrm{E}-03$ & $1.75 \mathrm{E}-03$ & $-2.87 \mathrm{E}-02$ \\
\hline $\mathrm{x}_{5}$ & * & $\mathrm{x}_{10}$ & $6.06 \mathrm{E}-02$ & $-1.95 \mathrm{E}+00$ & $-1.14 \mathrm{E}-02$ & $1.14 \mathrm{E}-01$ & $4.08 \mathrm{E}+01$ & $-4.99 \mathrm{E}-03$ & $-8.23 \mathrm{E}-04$ & $3.98 \mathrm{E}-02$ \\
\hline $\mathrm{x}_{5}$ & * & $\mathrm{x}_{11}$ & $-2.16 \mathrm{E}-03$ & 3.43E-01 & $6.03 \mathrm{E}-03$ & $3.58 \mathrm{E}-01$ & $-2.34 \mathrm{E}+01$ & $3.86 \mathrm{E}-03$ & $-5.80 \mathrm{E}-04$ & $-1.77 \mathrm{E}-02$ \\
\hline $\mathrm{x}_{5}$ & $*$ & $\mathrm{x}_{12}$ & $2.83 \mathrm{E}-03$ & $1.06 \mathrm{E}+00$ & $2.04 \mathrm{E}-03$ & $2.88 \mathrm{E}-01$ & $-8.97 \mathrm{E}+00$ & $-4.73 \mathrm{E}-05$ & $-3.36 \mathrm{E}-04$ & $1.38 \mathrm{E}-03$ \\
\hline $\mathrm{x}_{5}$ & $*$ & $\mathrm{x}_{13}$ & $1.69 \mathrm{E}-01$ & $2.74 \mathrm{E}+00$ & $-2.53 \mathrm{E}-02$ & $4.50 \mathrm{E}-01$ & $8.15 \mathrm{E}+01$ & $-4.30 \mathrm{E}-03$ & $-1.72 \mathrm{E}-03$ & $4.84 \mathrm{E}-02$ \\
\hline $\mathrm{x}_{5}$ & $*$ & $\mathrm{x}_{14}$ & $-5.45 \mathrm{E}-03$ & $-1.15 \mathrm{E}+00$ & $-4.22 \mathrm{E}-03$ & $-2.51 \mathrm{E}-01$ & $1.65 \mathrm{E}+01$ & $-1.12 \mathrm{E}-04$ & $5.05 \mathrm{E}-04$ & 4.33E-03 \\
\hline $\mathrm{x}_{6}$ & $*$ & $\mathrm{x}_{7}$ & $2.00 \mathrm{E}-01$ & 3.07E-01 & $-8.02 \mathrm{E}-03$ & $1.41 \mathrm{E}+00$ & $1.90 \mathrm{E}+01$ & $-9.51 \mathrm{E}-03$ & $1.28 \mathrm{E}-03$ & $3.93 \mathrm{E}-02$ \\
\hline $\mathrm{x}_{6}$ & $*$ & $\mathrm{x}_{8}$ & $-1.05 \mathrm{E}-01$ & $-1.30 \mathrm{E}+00$ & $3.79 \mathrm{E}-03$ & $-1.02 \mathrm{E}-01$ & $-2.60 \mathrm{E}+01$ & $-6.27 \mathrm{E}-03$ & $4.01 \mathrm{E}-03$ & $-3.13 \mathrm{E}-02$ \\
\hline $\mathrm{x}_{6}$ & $*$ & $\mathrm{x}_{9}$ & $-5.91 \mathrm{E}-02$ & $-9.13 \mathrm{E}-02$ & $1.75 \mathrm{E}-03$ & $-5.66 \mathrm{E}-02$ & $-4.75 \mathrm{E}+00$ & $-4.09 \mathrm{E}-04$ & $3.90 \mathrm{E}-04$ & $-7.18 \mathrm{E}-03$ \\
\hline $\mathrm{x}_{6}$ & * & $\mathrm{x}_{10}$ & $1.93 \mathrm{E}-03$ & $3.84 \mathrm{E}-01$ & $-8.90 \mathrm{E}-04$ & $-2.69 \mathrm{E}-02$ & $3.40 \mathrm{E}+00$ & $6.29 \mathrm{E}-05$ & 4.74E-07 & $3.96 \mathrm{E}-03$ \\
\hline $\mathrm{x}_{6}$ & * & $\mathrm{x}_{11}$ & 6.8 & +00 & -03 & $1.81 \mathrm{E}+00$ & $-5.33 \mathrm{E}+01$ & -2 & $1.66 \mathrm{E}-03$ & $1.52 \mathrm{E}-02$ \\
\hline $\mathrm{x}_{6}$ & * & $\mathrm{x}_{12}$ & 7.83E-01 & $1.35 \mathrm{E}+00$ & $-2.03 \mathrm{E}-02$ & $-1.54 \mathrm{E}+00$ & $5.08 \mathrm{E}+01$ & $1.63 \mathrm{E}-02$ & $-2.96 \mathrm{E}-03$ & $-8.18 \mathrm{E}-03$ \\
\hline $\mathrm{x}_{6}$ & $*$ & $\mathrm{x}_{13}$ & $-2.58 \mathrm{E}-02$ & $-2.20 \mathrm{E}+00$ & $2.62 \mathrm{E}-03$ & 1.17E-01 & $-3.12 \mathrm{E}+00$ & $-4.14 \mathrm{E}-04$ & $-4.73 \mathrm{E}-04$ & $-1.15 \mathrm{E}-02$ \\
\hline $\mathrm{x}_{6}$ & $*$ & $\mathrm{x}_{14}$ & $2.40 \mathrm{E}-01$ & $-3.39 \mathrm{E}+00$ & $-4.66 \mathrm{E}-03$ & $-3.26 \mathrm{E}-01$ & $2.06 \mathrm{E}+01$ & $5.47 \mathrm{E}-03$ & $-2.42 \mathrm{E}-03$ & $-7.96 \mathrm{E}-03$ \\
\hline $\mathrm{x}_{7}$ & 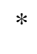 & $\mathrm{x}_{8}$ & $1.09 \mathrm{E}-02$ & 9.73E-02 & $-2.25 \mathrm{E}-05$ & $-3.54 \mathrm{E}-02$ & $-3.34 \mathrm{E}-01$ & $3.49 \mathrm{E}-04$ & $-1.66 \mathrm{E}-04$ & $-1.06 \mathrm{E}-03$ \\
\hline $\mathrm{x}_{7}$ & * & $\mathrm{X}_{9}$ & 7.53E-01 & $3.74 \mathrm{E}+00$ & 3.93E-03 & $2.73 \mathrm{E}+00$ & $-6.20 \mathrm{E}+00$ & $9.09 \mathrm{E}-03$ & $-2.64 \mathrm{E}-03$ & $6.18 \mathrm{E}-02$ \\
\hline$x_{7}$ & 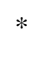 & $\mathrm{x}_{10}$ & $-7.83 \mathrm{E}-02$ & $3.20 \mathrm{E}+00$ & $1.42 \mathrm{E}-02$ & $1.27 \mathrm{E}-01$ & $-5.00 \mathrm{E}+01$ & $5.43 \mathrm{E}-03$ & 7.94E-04 & $-2.90 \mathrm{E}-02$ \\
\hline $\mathrm{x}_{7}$ & $*$ & $\mathrm{x}_{11}$ & $-2.12 \mathrm{E}-03$ & $-7.56 \mathrm{E}-01$ & $-1.07 \mathrm{E}-02$ & $-7.41 \mathrm{E}-01$ & $4.41 \mathrm{E}+01$ & $-7.95 \mathrm{E}-03$ & $7.86 \mathrm{E}-04$ & $3.00 \mathrm{E}-02$ \\
\hline
\end{tabular}


2021.

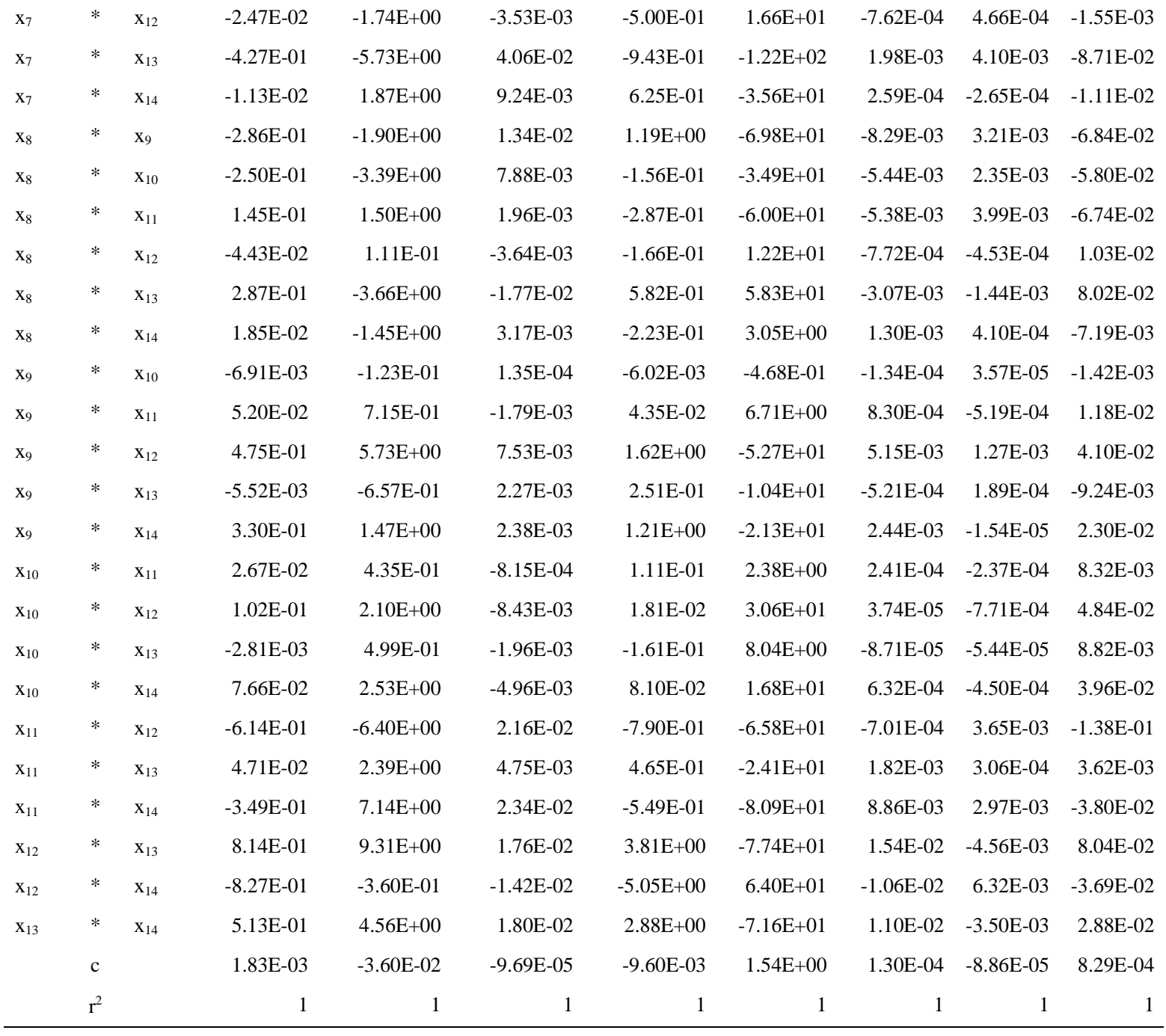

Table 8. The $\mathrm{x}$ and $\mathrm{y}$ variables, their coefficient and determination coefficient of equations belonging non-linear models yielded from the data collected Station 5

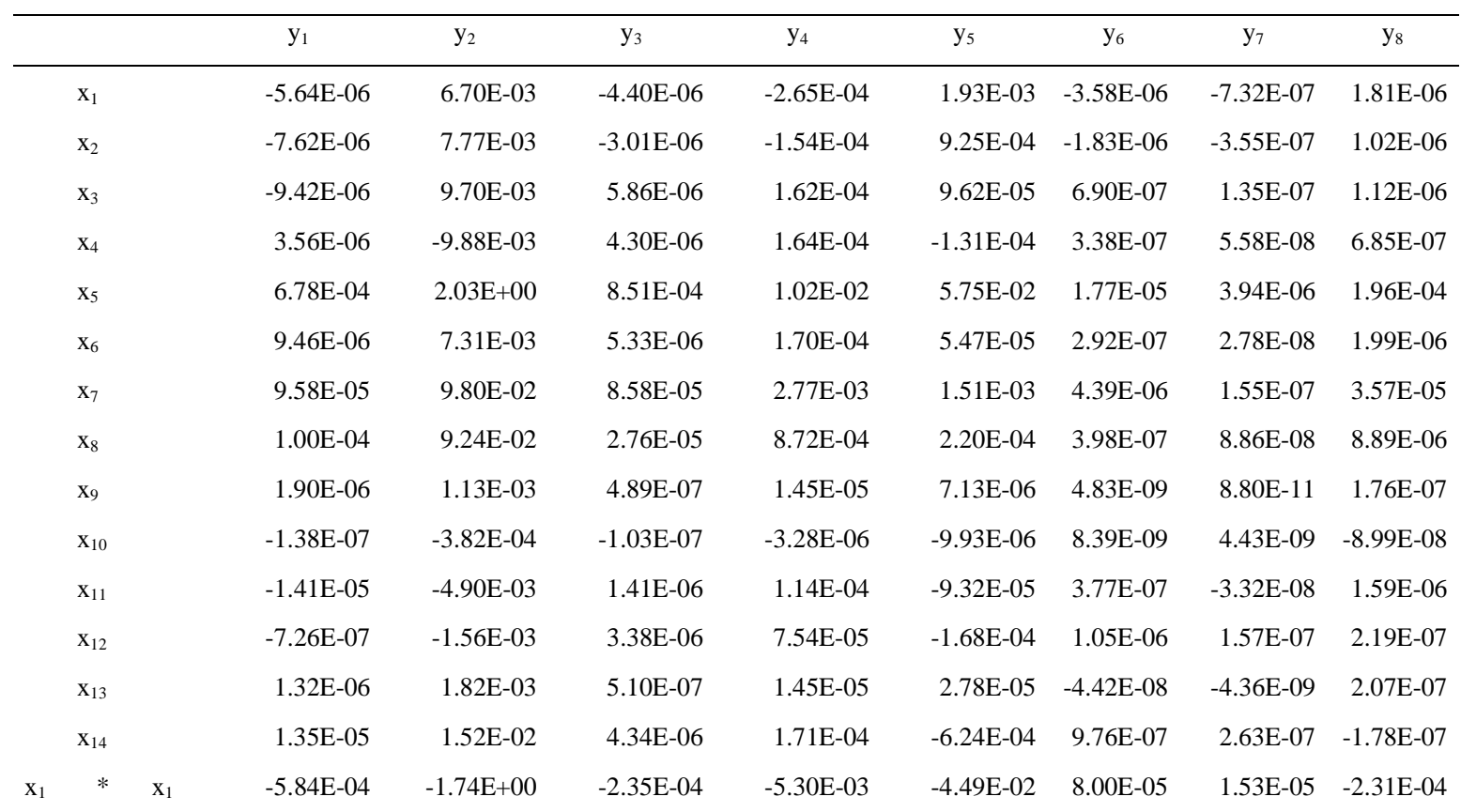


quality

\begin{tabular}{|c|c|c|c|c|c|c|c|c|c|c|}
\hline $\mathrm{x}_{2}$ & $*$ & $\mathrm{x}_{2}$ & $5.77 \mathrm{E}-07$ & $1.25 \mathrm{E}-03$ & $3.77 \mathrm{E}-07$ & 8.99E-06 & $1.22 \mathrm{E}-05$ & 4.77E-09 & $6.41 \mathrm{E}-09$ & $9.47 \mathrm{E}-08$ \\
\hline $\mathrm{x}_{3}$ & $*$ & $\mathrm{x}_{3}$ & $-1.34 \mathrm{E}-03$ & $-7.18 \mathrm{E}-01$ & $-1.77 \mathrm{E}-04$ & $-5.81 \mathrm{E}-03$ & $-1.62 \mathrm{E}-02$ & 4.46E-05 & $9.96 \mathrm{E}-06$ & $-1.51 \mathrm{E}-04$ \\
\hline $\mathrm{x}_{4}$ & $*$ & $\mathrm{x}_{4}$ & $-6.07 \mathrm{E}-04$ & $-5.85 \mathrm{E}-01$ & $-1.12 \mathrm{E}-05$ & $2.34 \mathrm{E}-04$ & $1.29 \mathrm{E}-03$ & $2.21 \mathrm{E}-06$ & $-2.81 \mathrm{E}-07$ & $-1.60 \mathrm{E}-05$ \\
\hline $\mathrm{x}_{5}$ & $*$ & $x_{5}$ & $-1.94 \mathrm{E}-09$ & $-8.18 \mathrm{E}-07$ & $-3.39 \mathrm{E}-09$ & 6.14E-09 & $-2.41 \mathrm{E}-07$ & $-1.04 \mathrm{E}-09$ & $8.88 \mathrm{E}-11$ & $-6.89 \mathrm{E}-10$ \\
\hline $\mathrm{x}_{6}$ & $*$ & $\mathrm{x}_{6}$ & $8.10 \mathrm{E}-05$ & $5.65 \mathrm{E}-02$ & $4.11 \mathrm{E}-05$ & $1.30 \mathrm{E}-03$ & $6.47 \mathrm{E}-04$ & $1.69 \mathrm{E}-06$ & $1.28 \mathrm{E}-07$ & $1.58 \mathrm{E}-05$ \\
\hline $\mathrm{x}_{7}$ & $*$ & $\mathrm{x}_{7}$ & $3.96 \mathrm{E}-05$ & $1.16 \mathrm{E}-01$ & 8.90E-06 & $4.26 \mathrm{E}-04$ & $-9.28 \mathrm{E}-04$ & 3.11E-06 & $6.42 \mathrm{E}-07$ & $-4.54 \mathrm{E}-06$ \\
\hline $\mathrm{x}_{8}$ & $*$ & $\mathrm{x}_{8}$ & $-1.59 \mathrm{E}-04$ & $-7.64 \mathrm{E}-02$ & $-6.54 \mathrm{E}-05$ & $-2.01 \mathrm{E}-03$ & $-1.26 \mathrm{E}-03$ & $-6.33 \mathrm{E}-06$ & $-2.20 \mathrm{E}-07$ & 3.94E-06 \\
\hline $\mathrm{x}_{9}$ & $*$ & $\mathrm{X}_{9}$ & $1.29 \mathrm{E}-06$ & 7.91E-04 & $3.75 \mathrm{E}-07$ & $1.02 \mathrm{E}-05$ & 1.13E-05 & 4.93E-09 & $-1.73 \mathrm{E}-10$ & $1.27 \mathrm{E}-07$ \\
\hline $\mathrm{x}_{10}$ & $*$ & $\mathrm{x}_{10}$ & 4.24E-08 & $-3.19 \mathrm{E}-05$ & $-5.41 \mathrm{E}-08$ & $-2.51 \mathrm{E}-06$ & $-7.43 \mathrm{E}-07$ & $-2.38 \mathrm{E}-09$ & $1.06 \mathrm{E}-09$ & $-4.10 \mathrm{E}-08$ \\
\hline $\mathrm{x}_{11}$ & $*$ & $\mathrm{x}_{11}$ & $-7.66 \mathrm{E}-05$ & $-2.48 \mathrm{E}-02$ & $6.43 \mathrm{E}-06$ & $5.70 \mathrm{E}-04$ & $-4.55 \mathrm{E}-04$ & $1.77 \mathrm{E}-06$ & $-1.98 \mathrm{E}-07$ & $8.10 \mathrm{E}-06$ \\
\hline$x_{12}$ & $*$ & $\mathrm{x}_{12}$ & $-1.46 \mathrm{E}-03$ & $-1.45 \mathrm{E}+00$ & $-2.79 \mathrm{E}-04$ & $-8.39 \mathrm{E}-03$ & $-3.43 \mathrm{E}-02$ & 7.92E-05 & $1.34 \mathrm{E}-05$ & $-2.02 \mathrm{E}-04$ \\
\hline $\mathrm{x}_{13}$ & $*$ & $\mathrm{x}_{13}$ & $9.04 \mathrm{E}-07$ & $2.13 \mathrm{E}-03$ & 3.83E-07 & $6.51 \mathrm{E}-06$ & $6.29 \mathrm{E}-05$ & $-1.02 \mathrm{E}-07$ & $-1.45 \mathrm{E}-08$ & $1.84 \mathrm{E}-07$ \\
\hline $\mathrm{x}_{14}$ & $*$ & $\mathrm{x}_{14}$ & $-5.11 \mathrm{E}-04$ & $-5.03 \mathrm{E}-01$ & $-1.70 \mathrm{E}-04$ & $-3.63 \mathrm{E}-03$ & $-3.98 \mathrm{E}-02$ & $5.81 \mathrm{E}-05$ & $1.43 \mathrm{E}-05$ & $-1.69 \mathrm{E}-04$ \\
\hline $\mathrm{x}_{1}$ & $*$ & $\mathrm{x}_{2}$ & $-3.15 \mathrm{E}-06$ & $2.77 \mathrm{E}-02$ & $5.71 \mathrm{E}-06$ & $7.72 \mathrm{E}-05$ & $1.08 \mathrm{E}-03$ & $-1.13 \mathrm{E}-06$ & $-1.72 \mathrm{E}-07$ & $2.99 \mathrm{E}-06$ \\
\hline $\mathrm{x}_{1}$ & $*$ & $\mathrm{x}_{3}$ & $-1.04 \mathrm{E}-03$ & $-1.11 \mathrm{E}+00$ & $-2.01 \mathrm{E}-04$ & $-5.74 \mathrm{E}-03$ & $-2.79 \mathrm{E}-02$ & $5.92 \mathrm{E}-05$ & $1.23 \mathrm{E}-05$ & $-1.82 \mathrm{E}-04$ \\
\hline $\mathrm{x}_{1}$ & $*$ & $\mathrm{x}_{4}$ & $5.09 \mathrm{E}-04$ & $-1.65 \mathrm{E}-01$ & $8.59 \mathrm{E}-05$ & $4.13 \mathrm{E}-03$ & $-1.29 \mathrm{E}-02$ & $1.65 \mathrm{E}-05$ & $3.12 \mathrm{E}-06$ & $1.11 \mathrm{E}-06$ \\
\hline $\mathrm{x}_{1}$ & $*$ & $\mathrm{x}_{5}$ & $-1.21 \mathrm{E}-05$ & $-3.10 \mathrm{E}-02$ & $-5.00 \mathrm{E}-06$ & $-2.18 \mathrm{E}-04$ & $-3.36 \mathrm{E}-05$ & $5.28 \mathrm{E}-07$ & $-2.19 \mathrm{E}-07$ & 5.37E-06 \\
\hline $\mathrm{x}_{1}$ & $*$ & $\mathrm{x}_{6}$ & $1.38 \mathrm{E}-04$ & $-8.08 \mathrm{E}-02$ & $3.72 \mathrm{E}-05$ & $1.35 \mathrm{E}-03$ & $-3.19 \mathrm{E}-03$ & $8.74 \mathrm{E}-06$ & $1.48 \mathrm{E}-06$ & $-2.17 \mathrm{E}-06$ \\
\hline $\mathrm{x}_{1}$ & $*$ & $\mathrm{x}_{7}$ & 3.32E-03 & $-1.71 \mathrm{E}+00$ & 4.97E-04 & $1.70 \mathrm{E}-02$ & $3.28 \mathrm{E}-03$ & $8.24 \mathrm{E}-06$ & $-3.29 \mathrm{E}-06$ & $2.96 \mathrm{E}-05$ \\
\hline $\mathrm{x}_{1}$ & $*$ & $\mathrm{x}_{8}$ & $2.43 \mathrm{E}-03$ & $1.55 \mathrm{E}+00$ & 7.92E-04 & 3.12E-02 & $-6.15 \mathrm{E}-02$ & $1.63 \mathrm{E}-04$ & $2.53 \mathrm{E}-05$ & 8.67E-05 \\
\hline $\mathrm{x}_{1}$ & $*$ & $\mathrm{X}_{9}$ & $4.98 \mathrm{E}-05$ & $1.65 \mathrm{E}-02$ & $8.84 \mathrm{E}-06$ & $2.93 \mathrm{E}-04$ & $-3.26 \mathrm{E}-05$ & $-1.40 \mathrm{E}-08$ & $-1.45 \mathrm{E}-08$ & $3.01 \mathrm{E}-06$ \\
\hline $\mathrm{x}_{1}$ & $*$ & $\mathrm{x}_{10}$ & $-3.06 \mathrm{E}-06$ & $-7.26 \mathrm{E}-03$ & $-1.11 \mathrm{E}-06$ & $-2.24 \mathrm{E}-05$ & $-1.44 \mathrm{E}-04$ & $1.35 \mathrm{E}-07$ & 4.92E-08 & $-9.25 \mathrm{E}-07$ \\
\hline $\mathrm{x}_{1}$ & $*$ & $\mathrm{x}_{11}$ & $-7.69 \mathrm{E}-05$ & $-3.49 \mathrm{E}-02$ & $1.13 \mathrm{E}-05$ & 8.59E-04 & $-6.13 \mathrm{E}-04$ & $2.40 \mathrm{E}-06$ & $-3.30 \mathrm{E}-07$ & $1.16 \mathrm{E}-05$ \\
\hline $\mathrm{x}_{1}$ & $*$ & $\mathrm{x}_{12}$ & $-1.08 \mathrm{E}-03$ & $-1.30 \mathrm{E}+00$ & $-2.16 \mathrm{E}-04$ & $-5.73 \mathrm{E}-03$ & $-3.58 \mathrm{E}-02$ & $7.30 \mathrm{E}-05$ & $1.41 \mathrm{E}-05$ & $-1.90 \mathrm{E}-04$ \\
\hline $\mathrm{x}_{1}$ & $*$ & $\mathrm{x}_{13}$ & 3.34E-05 & $2.04 \mathrm{E}-02$ & $6.70 \mathrm{E}-06$ & $1.65 \mathrm{E}-04$ & 3.87E-04 & $-5.92 \mathrm{E}-07$ & $-6.81 \mathrm{E}-08$ & $1.84 \mathrm{E}-06$ \\
\hline $\mathrm{x}_{1}$ & $*$ & $\mathrm{x}_{14}$ & $-6.63 \mathrm{E}-04$ & $-9.49 \mathrm{E}-01$ & $-1.80 \mathrm{E}-04$ & $-4.21 \mathrm{E}-03$ & $-3.76 \mathrm{E}-02$ & $6.47 \mathrm{E}-05$ & $1.42 \mathrm{E}-05$ & $-1.80 \mathrm{E}-04$ \\
\hline $\mathrm{x}_{2}$ & $*$ & $x_{3}$ & $-1.31 \mathrm{E}-05$ & $3.02 \mathrm{E}-02$ & $5.55 \mathrm{E}-06$ & 7.02E-05 & $1.10 \mathrm{E}-03$ & $-1.07 \mathrm{E}-06$ & $-1.44 \mathrm{E}-07$ & $2.70 \mathrm{E}-06$ \\
\hline $\mathrm{x}_{2}$ & $*$ & $\mathrm{x}_{4}$ & $-5.03 \mathrm{E}-06$ & $-7.98 \mathrm{E}-04$ & $2.73 \mathrm{E}-06$ & $6.64 \mathrm{E}-05$ & $2.20 \mathrm{E}-04$ & $-2.27 \mathrm{E}-07$ & $-4.45 \mathrm{E}-09$ & $5.81 \mathrm{E}-07$ \\
\hline $\mathrm{x}_{2}$ & $*$ & $\mathrm{x}_{5}$ & $2.84 \mathrm{E}-04$ & $-8.11 \mathrm{E}-01$ & $-1.36 \mathrm{E}-05$ & 8.23E-04 & $-3.45 \mathrm{E}-03$ & $1.51 \mathrm{E}-05$ & $2.67 \mathrm{E}-06$ & 7.19E-05 \\
\hline $\mathrm{x}_{2}$ & $*$ & $\mathrm{x}_{6}$ & $4.76 \mathrm{E}-06$ & $1.41 \mathrm{E}-02$ & 3.79E-06 & 7.95E-05 & $3.28 \mathrm{E}-04$ & $-2.63 \mathrm{E}-07$ & $-1.99 \mathrm{E}-08$ & $1.56 \mathrm{E}-06$ \\
\hline $\mathrm{x}_{2}$ & $*$ & $\mathrm{x}_{7}$ & $-4.38 \mathrm{E}-05$ & $2.88 \mathrm{E}-01$ & $3.98 \mathrm{E}-05$ & $-6.76 \mathrm{E}-05$ & $9.62 \mathrm{E}-03$ & $-1.15 \mathrm{E}-05$ & $-9.76 \mathrm{E}-07$ & $2.01 \mathrm{E}-05$ \\
\hline $\mathrm{x}_{2}$ & $*$ & $\mathrm{x}_{8}$ & $5.88 \mathrm{E}-05$ & $1.33 \mathrm{E}-01$ & $1.43 \mathrm{E}-06$ & $-3.96 \mathrm{E}-04$ & 3.34E-03 & $-6.30 \mathrm{E}-06$ & $-8.15 \mathrm{E}-07$ & $-2.02 \mathrm{E}-06$ \\
\hline $\mathrm{x}_{2}$ & $*$ & $\mathrm{x}_{9}$ & $9.18 \mathrm{E}-07$ & 1.26 & 07 & 06 & -05 & $-1.45 \mathrm{E}-08$ & $-7.17 \mathrm{E}-10$ & $1.23 \mathrm{E}-07$ \\
\hline $\mathrm{x}_{2}$ & $*$ & $\mathrm{x}_{10}$ & $-7.22 \mathrm{E}-08$ & $-5.45 \mathrm{E}-04$ & $-1.11 \mathrm{E}-07$ & $-2.30 \mathrm{E}-06$ & $-2.12 \mathrm{E}-05$ & $2.23 \mathrm{E}-08$ & 7.24E-09 & $-9.85 \mathrm{E}-08$ \\
\hline $\mathrm{x}_{2}$ & $*$ & $\mathrm{x}_{11}$ & $-9.32 \mathrm{E}-06$ & -2.65 & 97 & $6.90 \mathrm{H}$ & $-4.18 \mathrm{E}-05$ & $2.13 \mathrm{E}-07$ & $-2.20 \mathrm{E}-08$ & $1.03 \mathrm{E}-06$ \\
\hline $\mathrm{x}_{2}$ & $*$ & $\mathrm{x}_{12}$ & $-1.47 \mathrm{E}-05$ & $1.32 \mathrm{E}-02$ & $6.05 \mathrm{E}-07$ & $-8.45 \mathrm{E}-05$ & $9.13 \mathrm{E}-04$ & $-1.04 \mathrm{E}-06$ & $-1.75 \mathrm{E}-07$ & $9.68 \mathrm{E}-07$ \\
\hline $\mathrm{x}_{2}$ & $*$ & $\mathrm{x}_{13}$ & $E_{-} 06$ & E-03 & E-07 & E-06 & E-05 & $-7.80 \mathrm{E}-08$ & $-4.78 \mathrm{E}-09$ & $2.05 \mathrm{E}-07$ \\
\hline $\mathrm{x}_{2}$ & $*$ & $\mathrm{x}_{14}$ & 7.92E-06 & $3.28 \mathrm{E}-02$ & 4.09E-06 & 7.53E-05 & $4.08 \mathrm{E}-04$ & $-6.84 \mathrm{E}-07$ & $-1.09 \mathrm{E}-08$ & $1.31 \mathrm{E}-06$ \\
\hline $\mathrm{x}_{3}$ & $*$ & $\mathrm{x}_{4}$ & 2 & 2 & 55 & 3 & $-7.21 \mathrm{E}-03$ & $1.08 \mathrm{E}-05$ & 2.43E-06 & $1.48 \mathrm{E}-05$ \\
\hline $\mathrm{x}_{3}$ & $*$ & $\mathrm{x}_{5}$ & $2.63 \mathrm{E}-05$ & 1.16E-02 & $-4.40 \mathrm{E}-06$ & $-1.03 \mathrm{E}-04$ & $-7.17 \mathrm{E}-04$ & $-6.36 \mathrm{E}-07$ & $2.66 \mathrm{E}-07$ & $-2.12 \mathrm{E}-06$ \\
\hline $\mathrm{x}_{3}$ & $*$ & $\mathrm{x}_{6}$ & $-7.38 \mathrm{E}-05$ & $6.98 \mathrm{H}$ & 4.23E-05 & $1.18 \mathrm{E}-03$ & $1.15 \mathrm{E}-03$ & 4.18E-06 & 8.44E-07 & $9.11 \mathrm{E}-06$ \\
\hline $\mathrm{x}_{3}$ & $*$ & $\mathrm{x}_{7}$ & $-4.86 \mathrm{E}-03$ & $1.69 \mathrm{E}+00$ & 2.99E-04 & $1.24 \mathrm{E}-03$ & $1.26 \mathrm{E}-01$ & $-1.19 \mathrm{E}-04$ & $-2.01 \mathrm{E}-05$ & $2.24 \mathrm{E}-04$ \\
\hline $\mathrm{x}_{3}$ & $*$ & $\mathrm{x}_{8}$ & $2.02 \mathrm{E}-03$ & $2.78 \mathrm{E}+00$ & 8.79E-04 & $3.00 \mathrm{E}-02$ & $-1.06 \mathrm{E}-02$ & 8.39E-05 & $1.15 \mathrm{E}-05$ & $1.89 \mathrm{E}-04$ \\
\hline $\mathrm{x}_{3}$ & $*$ & $\mathrm{X}_{9}$ & $3.35 \mathrm{E}-05$ & $2.94 \mathrm{E}-02$ & $8.88 \mathrm{E}-06$ & $2.55 \mathrm{E}-04$ & $3.68 \mathrm{E}-04$ & $-4.77 \mathrm{E}-07$ & $-8.09 \mathrm{E}-08$ & $3.83 \mathrm{E}-06$ \\
\hline $\mathrm{x}_{3}$ & $*$ & $\mathrm{x}_{10}$ & $-3.73 \mathrm{E}-06$ & $-6.68 \mathrm{E}-03$ & $-1.18 \mathrm{E}-06$ & $-2.68 \mathrm{E}-05$ & $-1.52 \mathrm{E}-04$ & $1.61 \mathrm{E}-07$ & $6.02 \mathrm{E}-08$ & $-1.04 \mathrm{E}-06$ \\
\hline
\end{tabular}




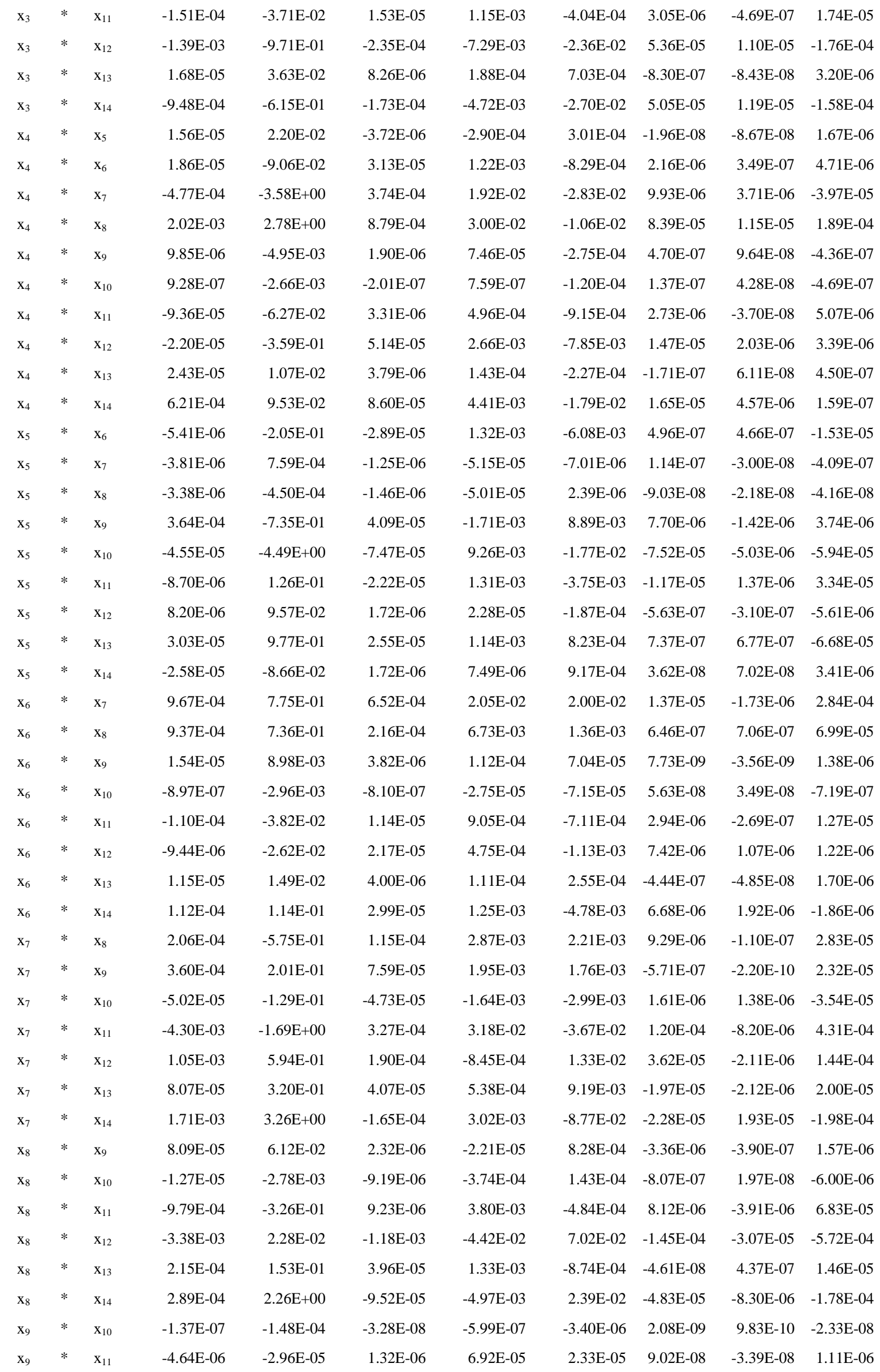



quality

\begin{tabular}{|c|c|c|c|c|c|c|c|c|c|c|}
\hline $\mathrm{X}_{9}$ & $*$ & $\mathrm{x}_{12}$ & $4.73 \mathrm{E}-05$ & $3.25 \mathrm{E}-02$ & $7.18 \mathrm{E}-06$ & $1.91 \mathrm{E}-04$ & $2.16 \mathrm{E}-04$ & $-6.03 E-07$ & $-7.81 \mathrm{E}-08$ & $3.16 \mathrm{E}-06$ \\
\hline $\mathrm{X}_{9}$ & $*$ & $\mathrm{x}_{13}$ & $8.52 \mathrm{E}-07$ & $8.48 \mathrm{E}-04$ & $2.80 \mathrm{E}-07$ & 7.36E-06 & $1.75 \mathrm{E}-05$ & $-2.20 \mathrm{E}-08$ & $-3.40 \mathrm{E}-09$ & $1.12 \mathrm{E}-07$ \\
\hline $\mathrm{x}_{9}$ & $*$ & $\mathrm{x}_{14}$ & $4.45 \mathrm{E}-05$ & $3.40 \mathrm{E}-02$ & 7.59E-06 & 2.31E-04 & $5.98 \mathrm{E}-05$ & $-4.63 \mathrm{E}-07$ & $-2.84 \mathrm{E}-08$ & $2.97 \mathrm{E}-06$ \\
\hline $\mathrm{x}_{10}$ & $*$ & $\mathrm{x}_{11}$ & $-2.14 \mathrm{E}-06$ & $-1.12 \mathrm{E}-03$ & 8.39E-08 & $1.34 \mathrm{E}-05$ & $-3.25 \mathrm{E}-05$ & 7.43E-08 & 2.24E-09 & $1.24 \mathrm{E}-07$ \\
\hline $\mathrm{x}_{10}$ & $*$ & $\mathrm{x}_{12}$ & $-2.43 \mathrm{E}-06$ & $-7.35 \mathrm{E}-03$ & $-1.55 \mathrm{E}-06$ & $-3.73 \mathrm{E}-05$ & $-1.93 \mathrm{E}-04$ & $1.62 \mathrm{E}-07$ & $6.49 \mathrm{E}-08$ & $-1.23 \mathrm{E}-06$ \\
\hline $\mathrm{x}_{10}$ & $*$ & $\mathrm{x}_{13}$ & $1.38 \mathrm{E}-07$ & $-1.39 \mathrm{E}-04$ & $-6.27 \mathrm{E}-08$ & $-2.08 \mathrm{E}-06$ & $-8.62 \mathrm{E}-06$ & $1.66 \mathrm{E}-09$ & 3.84E-09 & $-6.21 \mathrm{E}-08$ \\
\hline $\mathrm{x}_{10}$ & $*$ & $\mathrm{x}_{14}$ & $-2.14 \mathrm{E}-06$ & $-6.56 \mathrm{E}-03$ & $-1.46 \mathrm{E}-06$ & $-3.52 \mathrm{E}-05$ & $-2.08 \mathrm{E}-04$ & $1.85 \mathrm{E}-07$ & 7.55E-08 & $-1.30 \mathrm{E}-06$ \\
\hline $\mathrm{x}_{11}$ & $*$ & $\mathrm{x}_{12}$ & $-9.75 \mathrm{E}-05$ & $-3.77 \mathrm{E}-02$ & $1.29 \mathrm{E}-05$ & $1.08 \mathrm{E}-03$ & $-1.34 \mathrm{E}-03$ & $3.87 \mathrm{E}-06$ & $-2.72 \mathrm{E}-07$ & $1.51 \mathrm{E}-05$ \\
\hline $\mathrm{X}_{11}$ & $*$ & $\mathrm{x}_{13}$ & $-1.28 \mathrm{E}-05$ & $-3.68 \mathrm{E}-03$ & $9.70 \mathrm{E}-07$ & $8.55 \mathrm{E}-05$ & $-4.70 \mathrm{E}-05$ & $2.34 \mathrm{E}-07$ & $-3.53 \mathrm{E}-08$ & $1.24 \mathrm{E}-06$ \\
\hline $\mathrm{x}_{11}$ & $*$ & $\mathrm{x}_{14}$ & $-1.40 \mathrm{E}-04$ & $-3.95 \mathrm{E}-02$ & $1.26 \mathrm{E}-05$ & $1.20 \mathrm{E}-03$ & $-1.47 \mathrm{E}-03$ & $3.84 \mathrm{E}-06$ & $-3.25 \mathrm{E}-07$ & $1.62 \mathrm{E}-05$ \\
\hline $\mathrm{x}_{12}$ & $*$ & $\mathrm{x}_{13}$ & $3.10 \mathrm{E}-05$ & $3.92 \mathrm{E}-02$ & $6.02 \mathrm{E}-06$ & $1.12 \mathrm{E}-04$ & 5.32E-04 & $-1.19 \mathrm{E}-06$ & $-8.78 \mathrm{E}-08$ & $2.36 \mathrm{E}-06$ \\
\hline $\mathrm{x}_{12}$ & $*$ & $\mathrm{x}_{14}$ & $-8.94 \mathrm{E}-04$ & $-8.84 \mathrm{E}-01$ & $-2.38 \mathrm{E}-04$ & $-6.26 \mathrm{E}-03$ & $-3.80 \mathrm{E}-02$ & $6.31 \mathrm{E}-05$ & $1.37 \mathrm{E}-05$ & $-1.90 \mathrm{E}-04$ \\
\hline \multirow[t]{3}{*}{$x_{13}$} & $*$ & $\mathrm{X}_{14}$ & $3.38 \mathrm{E}-05$ & 4.13E-02 & $7.26 \mathrm{E}-06$ & $1.98 \mathrm{E}-04$ & $1.91 \mathrm{E}-04$ & $-7.29 \mathrm{E}-07$ & 8.73E-09 & $2.28 \mathrm{E}-06$ \\
\hline & $\mathrm{c}$ & & $1.10 \mathrm{E}-06$ & $9.50 \mathrm{E}-04$ & $6.90 \mathrm{E}-07$ & $2.21 \mathrm{E}-05$ & 3.89E-06 & 4.60E-08 & 4.84E-09 & $2.50 \mathrm{E}-07$ \\
\hline & $r^{2}$ & & 0.594 & 0.469 & 0.07 & 0.464 & 0.315 & 0.628 & 0.51 & 0.507 \\
\hline
\end{tabular}

As observed from the tables, higher determination coefficients were obtained from the nonlinear equations. The other situation which should be taken into account is that the $\mathrm{R}^{2}$ values of the equations yielded from the data collected from fresh water stations $\left(1^{\text {st }}, 3^{\text {th }}\right.$ and $4^{\text {th }}$ stations) equal 1 . The second and the fifth stations are the delta region of Samanlı and Safran Rivers, respectively. This situation can be explained by the fact that the interferring effect of the complex matrics and dynamic medium of delta regions. In order to inspect conformity of the experimental and theoretical values of each parameters, theoretical data were calculated for all samples for each week by using these linear and nonlinear equations. The representative conformity graphs were drawn for dissolved oxygen, suspended solid, nitrite, nitrate and phosphate parameters, and the first and fourth stations. The consistence of the experimental and theoretical values was introduced in Figures 2a-e and Figures 3a-e.

DO

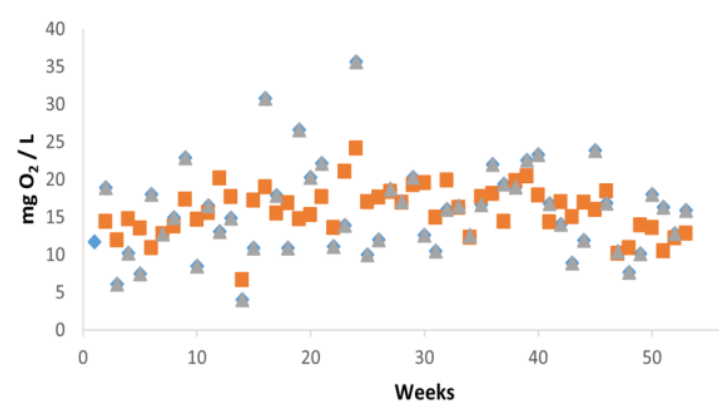

(a) ss

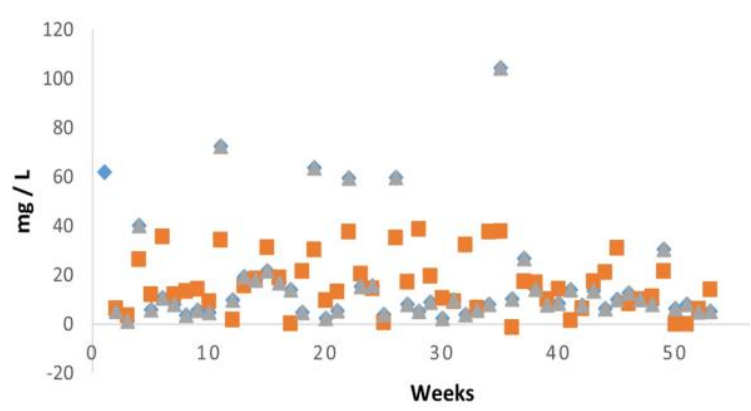

(b) 


\section{Nitrate}

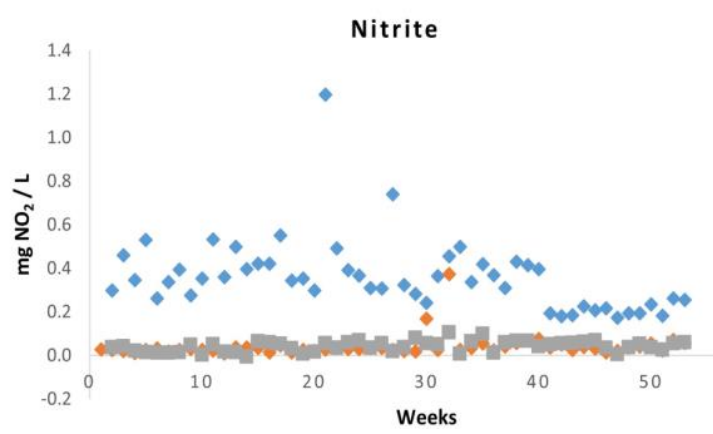

(c)

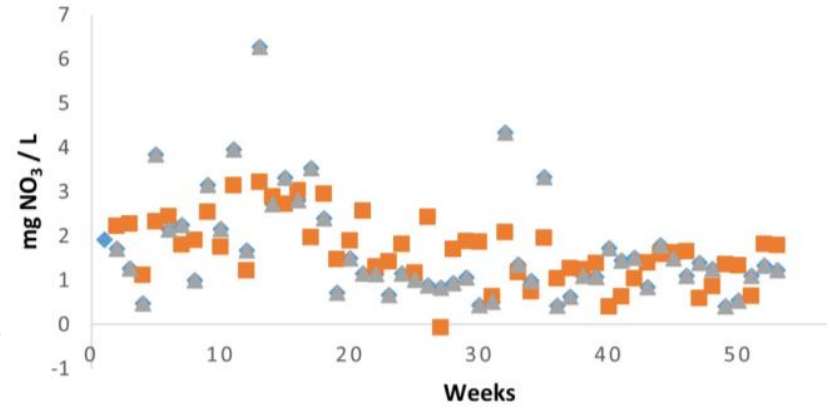

(d)

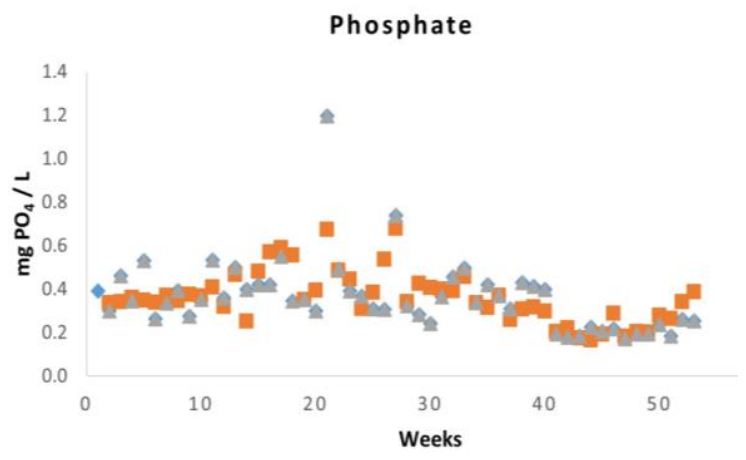

Experimental Results $\quad$ Linear Model Results $\Delta$ Nonlinear Model Results

(e)

Figure 2. The conformity of the experimental and theoretical values for a) dissolved oxygen, b) suspended solid, c) nitrite, d) nitrate, e) phosphate parameters of the water samples collected from the first sitation.

DO

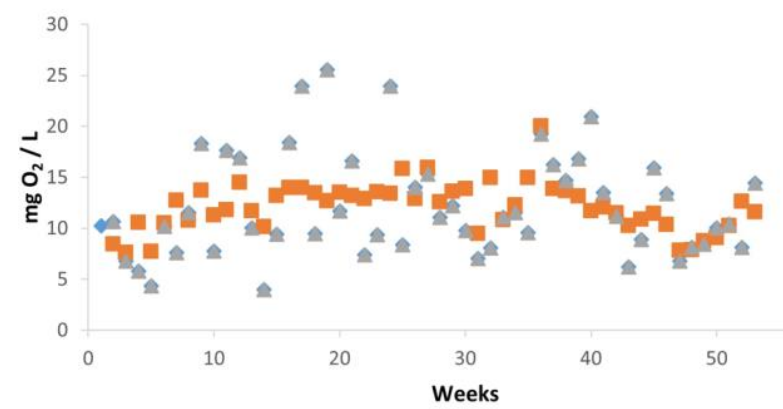

(a)

Nitrite

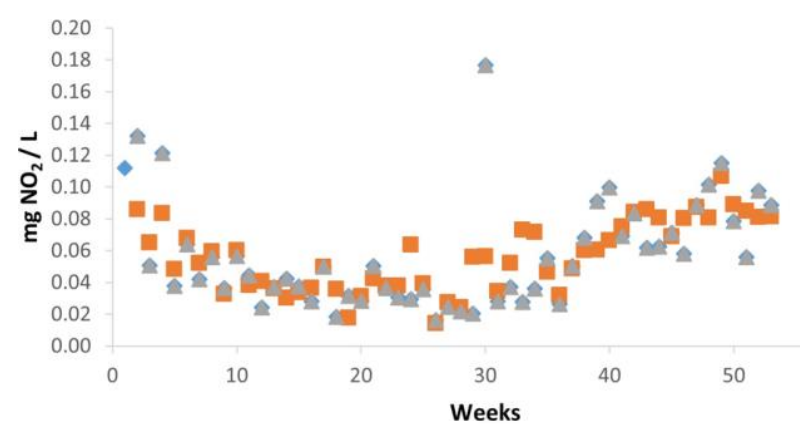

(c)

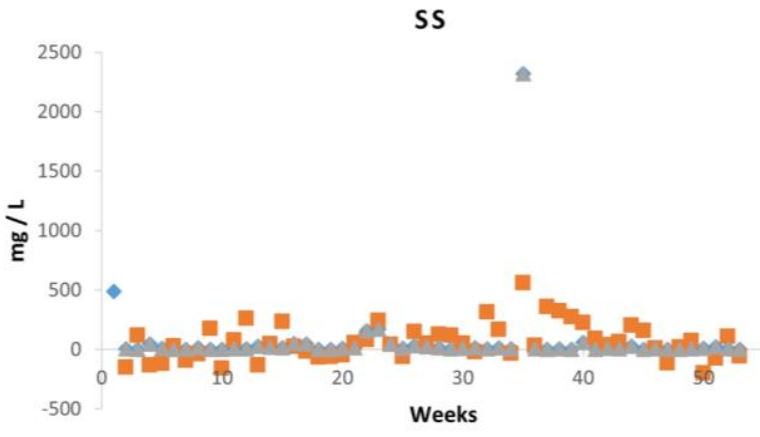

(b)

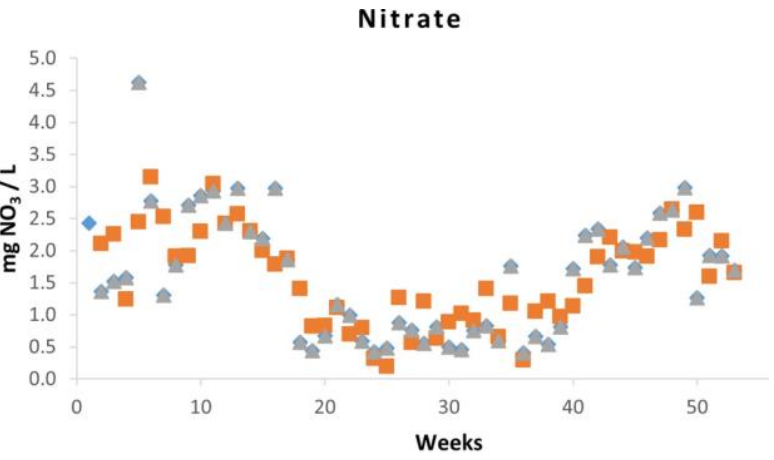

(d) 


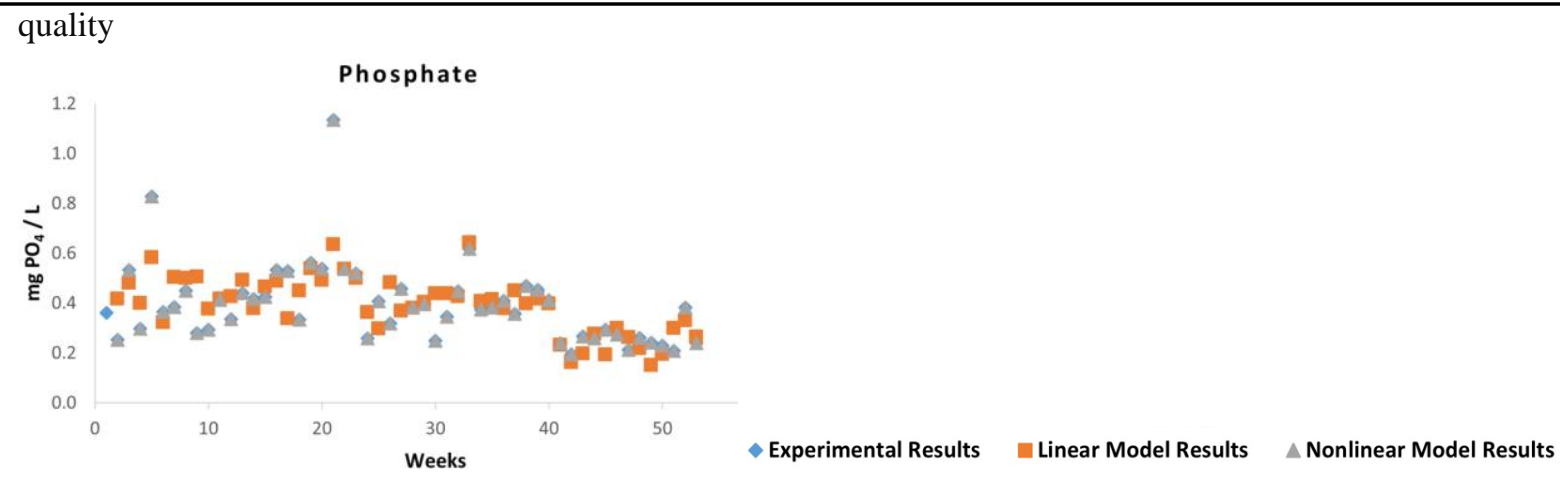

(e)

Figure 3. The conformity of the experimental and theoretical values for a) dissolved oxygen, b) suspended solid, c) nitrite, d) nitrate, e) phosphate parameters of the water samples collected from the fourth station

As seen from the figures, both linear and nonlinear models conform well the experimental results. Especially, nonlinear equations gave satisfactory results with high determination coefficient. Additionally, as seen from the figures, at least eight months estimation can be performed almost certainly for fresh water samples. Thus, these equations can be used for determination of quality parameters This situation shows that the usage of these model equations allows the determination of tedious quality parameters such as nitrite, nitrate and phosphate without doing any analysis.

\section{CONCLUSIONS}

The water quality of the Samanlı and Safran Rivers in Yalova were examined in terms of physicochemical parameters, alkalinity and nitrate, nitrite, phosphate parameters, in order to monitor water quality by the aid of forecasting models. As a result, it was determined that the linear models predicted value of parameters for next 8 months. The models works well especially for the samples collected from fresh water. Consequently, it can be said that these models can be used for readily forecasting quality parameters requiring effort and time to perform.

\section{ACKNOWLEDGMENT}

This study was supported with Yalova University BAP Master Thesis Project (2018/YL/0014).

\section{REFERENCES}

[1] Singh KP, Malik A, S. Sinha S (2005) Water quality assessment and apportionment of pollution sources of Gomti river (India) using multivariate statistical techniques - A case study. Anal Chim Acta 538:355374. https://doi.org/10.1016/j.aca.2005.02.006

[2] Shrestha S, Kazama F (2007) Assessment of surface water quality using multivariate statistical techniques: A case study of the Fuji river basin, Japan. Environ Model Softw 22:464-475. https://doi.org/10.1016/j.envsoft.2006.02.001

[3] Singh KP, Malik A, Mohan D, Sinha S (2004) Multivariate statistical techniques for the evaluation of spatial and temporal variations in water quality of Gomti River (India) - A case study. Water Res 38: 
[4] Nguyen TH, Helm B, Hettiarachchi H, Caucci S, Krebs P (2019) The selection of design methods for river water quality monitoring networks: a review. Environ Earth Sci 78:1-17. https://doi.org/10.1007/s12665-019-8110-x

[5] Villas-boas MD, Olivera F, De Azevedo JPS (2017) Assessment of the water quality monitoring network of the Piabanha River experimental watersheds in Rio de Janeiro, Brazil, using autoassociative neural networks. Environ Monit Assess 189: 439. https://doi.org/10.1007/s10661-017-6134-9

[6] Mccaffrey S (2012) Water quality parameters \& indicator. https://sswm.info/node/4300 Accessed 15 $\underline{\text { June } 2021}$

[7] Yang G, Moyer DL (2020) Estimation of nonlinear water-quality trends in high-frequency monitoring data. Sci Total Environ 715:136686. https://doi.org/10.1016/j.scitotenv.2020.136686

[8] Juntunen P, Liukkonen M, Pelo M, Lehtola MJ, Hiltunen Y (2012) Modelling of water quality: an application to a water treatment process. Appl Comput Intell Soft Comput 2012:1-9. https://doi.org/10.1155/2012/846321

[9] Maqbool F, Malik AH, Bhatti ZA, Pervez A, Suleman M (2012) Application of regression model on stream water quality parameters. Pakistan J Agric Sci vol. 49:95-100

[10] El-Korashey R (2009) Using regression analysis to estimate water quality constituents in Bahr El Baqar drain. J Appl Sci Res 5:1067-1076

[11] Mustapha A, Abdu A (2012) Application of principal component analysis \& multiple regression models in surface water quality assessment. J Environ Earth Sci 2:16-23

[12] Majeed SAAD, Saleh LAM, Aswed GK (2018) Modeling the water quality index and climate variables using an artificial neural network and non-linear regression. Int J Eng Technol 7:1346-1350. https://doi.org/10.14419/ijet.v7i3.9519

[13] Yalova Çevre ve Şehircilik İl Müdürlüğü (2016) Yalova İli 2015 yılı çevre durum raporu. [Online]. Available: https://yalova.csb.gov.tr/

[14] U.S. Environmental Protection Agency (1982) Method 150.2: pH, continuous monitoring (electrometric) by $\mathrm{pH}$ meter. [Online]. Available: www.epa.gov

[15] U.S. Environmental Protection Agency (1982) Method 120.1: Conductance (specific conductance, $\mu$ mhos at $25^{\circ} \mathrm{C}$ ) by conductivity meter

[16] U.S. Environmental Protection Agency (1971) Method 360.1 Oxygen, dissolved (membrane electrode).

[17] U.S. Environmental Protection Agency (1978) Alkalinity (titrimetric, pH 4.5).

[18] U.S. Environmental Protection Agency (1971) Residue, non-filterable (gravimetric, dried at $103-105^{\circ} \mathrm{C}$ ).

[19] U.S. Environmental Protection Agency (1974) Nitrogen, nitrate-nitrite (spectrophotometric, cadmium reduction).

[20] U.S. Environmental Protection Agency (1978) Method 365.3: Phosphorous, all forms (colorimetric, ascorbic acid, two reagent).

[21] Amini H, Taghavi-Shahri SM, Henderson SB, Naddafi K, Nabizadeh R, Yunesian M (2014) Land use regression models to estimate the annual and seasonal spatial variability of sulfur dioxide and particulate matter in Tehran, Iran. Sci Total Environ 488-489: 343-353. https://doi.org/10.1016/j.scitotenv.2014.04.106

[22] More AG, Gupta SK (2018) Predictive modelling of chromium removal using multiple linear and nonlinear regression with special emphasis on operating parameters of bioelectrochemical reactor. J Biosci Bioeng 126:205-212. https://doi.org/10.1016/j.jbiosc.2018.02.013 
[23] Ramesh N, Ramesh S, Vennila G, Abdul Bari J, MageshKumar P (2016) Energy production through organic fraction of municipal solid waste-A multiple regression modeling approach. Ecotoxicol Environ Saf 134:350-357. https://doi.org/10.1016/j.ecoenv.2015.08.027

[24] Kicsiny R (2018) Black-box model for solar storage tanks based on multiple linear regression. Renew Energy 125:857-865. https://doi.org/10.1016/j.renene.2018.02.037

[25] Salem S, Berends D, Heijnen JJ, Van Loosdrecht MCM (2002) Model-based evaluation of a new upgrading concept for N-removal. Water Sci Technol 45:169-176. https://doi.org/10.2166/wst.2002.0104 